\title{
Econophysics of cryptocurrency crashes: an overview
}

\author{
Andrii Bielinskyi ${ }^{1, *}$, Oleksandr Serdyuk ${ }^{2, * *}$, Serhiy Semerikov ${ }^{1,3,4,5, * * *}$, and Vladimir Soloviev Si,2,*** $^{1, *}$ \\ ${ }^{1}$ Kryvyi Rih State Pedagogical University, 54 Gagarin Ave., Kryvyi Rih, 50086, Ukraine \\ ${ }^{2}$ The Bohdan Khmelnytsky National University of Cherkasy, 81 Shevchenko Blvd., Cherkasy, 18031, Ukraine \\ ${ }^{3}$ Kryvyi Rih National University, 11 Vitalii Matusevych Str., Kryvyi Rih, 50027, Ukraine \\ ${ }^{4}$ Institute of Information Technologies and Learning Tools of the NAES of Ukraine, 9 M. Berlynskoho Str., Kyiv, 04060, Ukraine \\ ${ }^{5}$ University of Educational Management, 52-A Sichovykh Striltsiv Str., Kyiv, 04053, Ukraine
}

\begin{abstract}
Cryptocurrencies refer to a type of digital asset that uses distributed ledger, or blockchain technology to enable a secure transaction. Like other financial assets, they show signs of complex systems built from a large number of nonlinearly interacting constituents, which exhibits collective behavior and, due to an exchange of energy or information with the environment, can easily modify its internal structure and patterns of activity. We review the econophysics analysis methods and models adopted in or invented for financial time series and their subtle properties, which are applicable to time series in other disciplines. Quantitative measures of complexity have been proposed, classified, and adapted to the cryptocurrency market. Their behavior in the face of critical events and known cryptocurrency market crashes has been analyzed. It has been shown that most of these measures behave characteristically in the periods preceding the critical event. Therefore, it is possible to build indicators-precursors of crisis phenomena in the cryptocurrency market.
\end{abstract}

\section{Introduction}

The instability of global financial systems concerning normal and natural disturbances of the modern market and the presence of poorly foreseeable financial crashes indicate, first of all, the crisis of the methodology of modeling, forecasting, and interpretation of modern socio-economic realities. The doctrine of the unity of the scientific method states that for the study of events in socio-economic systems, the same methods and criteria as those used in the study of natural phenomena are applicable. Rapidly evolving coronavirus pandemic brings a devastating effect on the entire world and its economy as a whole [1-7]. Further instability related to COVID-19 will negatively affect not only on companies and financial markets, but also on traders and investors that have been interested in saving their investment, minimizing risks, and making decisions such as how to manage their resources, how much to consume and save, when to buy or sell stocks, etc., and these decisions depend on the expectation of when to expect next critical change [8-21]. Despite the complexity of the problem, the results of recent studies indicate that significant success has been achieved within the framework of interdisciplinary approaches, and the theory of selforganization - synergetics $[22,23]$. The modern paradigm of synergetics is a complex paradigm associated with the possibility of direct numerical simulation of the processes of complex systems evolution, most of which have a net-

\footnotetext{
*e-mail: krivogame@gmail.com

**e-mail: serdyuk@ukr.net

***e-mail: semerikov@gmail.com

****e-mail: vnsoloviev2016@gmail.com
}

work structure, or one way or another can be reduced to the network. The theory of complex networks studies the characteristics of networks, taking into account not only their topology but also statistical properties, the distribution of weights of individual nodes and edges, the effects of dissemination of information, robustness, etc. [1-4, 2426].

Complex systems consist of a plurality of interacting agents possessing the ability to generate new qualities at the level of macroscopic collective behavior, the manifestation of which is the spontaneous formation of noticeable temporal, spatial, or functional structures [27-32]. As simulation processes, the application of quantitative methods involves measurement procedures, where importance is given to complexity measures. Prigogine notes that the concepts of simplicity and complexity are relativized in the pluralism of the descriptions of languages, which also determines the plurality of approaches to the quantitative description of the complexity phenomenon [5].

Financial markets have been attracting the attention of many scientists like engineers, mathematicians, physicists, and others for the last two decades. Such vast interest transformed into a branch of statistical mechanics -econophysics [30-34]. Physics, economics, finance, sociology, mathematics, engineering, and computer science are fields which, as a result of cross-fertilization, have created the multi-, cross-, and interdisciplinary areas of science and research such as econophysics and sociophysics, thriving in the last two and a half decades. These mixed research fields use knowledge, methodologies, methods, and tools of physics for modeling, explaining and forecasting 
economic, social phenomena, and processes. Accordingly, econophysics is an interdisciplinary research field, applying theories and methods originally developed by physicists to solve problems in economics, usually those including uncertainty or stochastic processes, nonlinear dynamics, and evolutionary games.

There are deep relationships (as well as crucial differences) between physics and finance [35] that have inspired generations of physicists as well as economists. In general, physicists apprehend financial markets as complex systems and, as such, they conducted numerous scientific investigations [36].

Though statistical physics cannot get along without quantum-mechanical ideas and notions in its fundamentals, the main sphere of its interest is the macroscopic description of systems with a large number of particles, the dynamic behavior of which can't be brought to microscopic dynamical equations of quantum mechanics figured out for separate particles without the use of respective statistical postulates [37]. During last years an increasing flow of works was observed, in which detailed models of market process participants interactions and quantummechanical analogies, notions, and terminology based on methods of describing socio-economic systems are drawn to explain both particular peculiarities of modern market dynamics and economic functioning in whole [38-40]. In papers [41, 42], Soloviev and Saptsin have suggested a new paradigm of complex systems modeling based on the ideas of quantum as well as relativistic mechanics. It has been revealed that the use of quantum-mechanical analogies (such as the uncertainty principle, the notion of the operator, and quantum measurement interpretation) can be applied for describing socio-economic processes.

In this review, we will continue to study Prigogine's manifestations of the system complexity, using the current methods of quantitative analysis to determine the appropriate measures of complexity. The proposed measures of complexity, depending on the methodology and construction methods, can be divided into the following classes:

(1) informational,

(2) (multi-)fractal,

(3) chaos-dynamic,

(4) recurrent,

(5) irreversible,

(6) based on complex networks,

(7) quantum.

Econophysics, based on a rich arsenal of research on critical phenomena [43], very successfully copes with the description of similar events in economics and finance. These are crises and crashes that are constantly shaking the world economy. The introduced measures of complexity should, to one degree or another, respond to such phenomena.

The key idea here is the hypothesis that the complexity of the system before the crashes and the actual periods of crashes must change. This should signal the corresponding degree of complexity if they are able to quantify certain patterns of a complex system. A significant advantage of the introduced measures is their dynamism, that is, the ability to monitor the change in time of the chosen measure and compare it with the corresponding dynamics of the output time series. This allowed us to compare the critical changes in the dynamics of the system, which is described by the time series, with the characteristic changes of concrete measures of complexity. It turned out that quantitative measures of complexity respond to critical changes in the dynamics of a complex system, which allows them to be used in the diagnostic process and prediction of future changes.

The cryptocurrency market is a complex, selforganized system, which in most cases can be considered either as a complex network of market agents or as an integrated output signal of this network - a time series, for example, prices of individual cryptocurrency. The research on cryptocurrency price fluctuations being carried out internationally is complicated due to the interplay of many factors - including market supply and demand, the US dollar exchange rate, stock market state, the influence of crime, shadow market, and fiat money regulator pressure that introduces a high level of noise into the cryptocurrency data. Moreover, in the cryptocurrency market, to some extent, blockchain technology is tested in general. Hence, the cryptocurrency prices exhibit such complex volatility characteristics as nonlinearity and uncertainty, which are difficult to forecast, and any obtained results are uncertain. Therefore, cryptocurrency price prediction remains a huge challenge [44-58].

As can be seen, markets have seen significant numbers of investors selling off and rebalancing their portfolios with less risky assets. That has been leading to large losses and high volatility, typical of crisis periods. The economy key for preventing such activity may lie in cryptocurrency and constructing effective indicators of possible critical states that will help investors and traders fill in safety. Bitcoin, which is associated with the whole crypto market, has such properties as detachment and independence from the standard financial market and the proclaimed properties that should make it serve as the digital gold [59]. As was shown by Kristoufek [60], Bitcoin promises to be a safe-haven asset with its low correlation with gold, S\&P 500, Dow Jones Industrial Average, and other authoritative stock indices even in the extreme events. But authors please not overestimate the cryptocurrency since according to their calculations and, obviously, the current structure of the system, gold remains more significant. But for ten years, this token has been discussed by many people, it has experienced a lot in such a short period, many people believe in it, and it has managed to form a fairly complex and self-organized system. The integrated actions from real-world merge in such dynamics and relevant information that is encoded in Bitcoin's time series can be extracted [61-63]. In the context of volatile financial markets, it is important to select such measures of complexity that will be able to notify us of upcoming abnormal events in the form of crises at an early stage. 
In this review we:

- present such measures;

- study critical and crash phenomena that have taken place in the cryptocurrency market;

- try to understand whether crashes and critical events could be identified and predicted by such informative indicators or not.

This review is dedicated to the construction of such indicators based on the theory of complexity. According to our goals and actions, the paper is structured as follows. In Section 2, we present our classification of Bitcoin's crises for the period from July 16, 2010 to January 21, 2021. In Section 3, we describe the information measures of complexity. In Section 4, we describe the multifractal analysis methodology and its results for the crypto market. Section 5 defines what is chaos-based measures of complexity. In section 6 , we deal with the recurrence quantification analysis of critical and crisis phenomena in the cryptocurrency market. Irreversible measure based on permutation patterns is defined in Section 7. Section 8 presents the theory and empirical results on network and multiplex measures of complexity and their robustness for digital currencies. Section 9 defines quantum complexity measures, the features of their manifestation on the crypto market are discussed. Section 10 contains conclusions and some recommendations for further research.

\section{Data and classification}

Bitcoin, being the most capitalized cryptocurrency, as a rule, sets and determines the main trends of the crypto market as a whole. Therefore, except for the part of the work where the study of collective processes in the market is carried out, we will use the time series of Bitcoin [64]. From figure 1 it can be seen that at the beginning of its existence, Bitcoin's dynamic was determined mainly by the processes of the formation of the market as a whole and characterized by high volatility, which, however, was not associated with critical phenomena. Bariviera et al. [65] find that the Hurt exponent changes significantly during the first years of existence of Bitcoin, and now it is less unstable than before. Moreover, they detect more evidence of information since 2014 [66].

Being historically proven, popular, and widely used cryptocurrency for the whole existence of cryptocurrencies in general, Bitcoin began to produce a lot of news and speculation, which began to determine its future life. Similar discussions began to lead to different kinds of crashes, critical events, and bubbles, which professional investors and inexperienced users began to fear. Thus, we advanced into action and set the tasks:

- classification of such critical events and crashes;

- construction of such indicators that will predict crashes, critical events in order to allow investors and ordinary users to work in this market.

Accordingly, during this period in the Bitcoin market, many crashes and critical events shook it. At the moment,

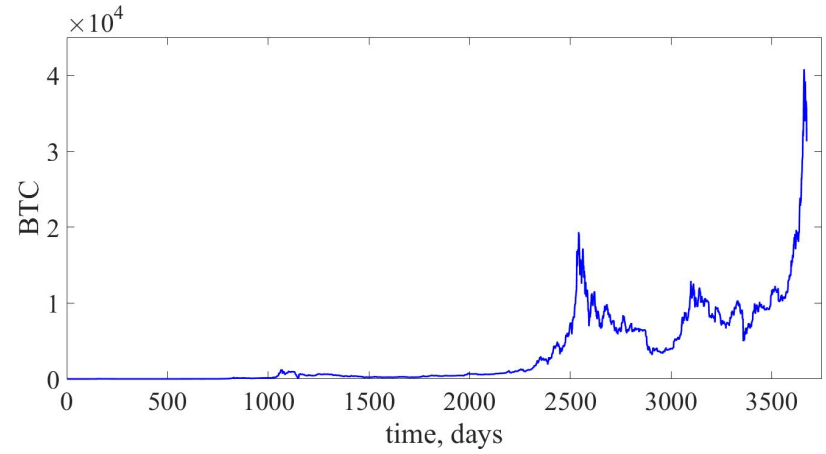

Figure 1: Bitcoin price development from July 16, 2010 to January 21, 2021

there are various research works on what crashes are and how to classify such risk events in the market. The definition of these events still has been debatable. Nevertheless, the proposals of most authors have common elements that allow us to arrive at a consensus. Generally, the market crash is a sudden drastic decline in the prices of financial assets in a specific market [67]. Additionally, the applied model for a specific market takes an important place in the definition of "drastic decline". For instance, Wang et al. [67] take into account events with a minimum one-day decrease of $5 \%$ in the stock returns. These authors [26] identify financial crashes as a decrease of $25 \%$ or less of multi-year financial returns. Lleo and Ziembda [68] define a crash as a specific event of a day, which decreasing closing price exceeds a fall of $10 \%$ between the highest and the lowest value of the stock index in a year. Hong and Stein [69] postulate that the market crash is an unexpected event in which appearance was not accompanied by any financial news. Moreover, the price change during this event is rather negative. Also, it is worth mentioning the study of Min Shu and Wei Zhu [70] where their classification of crashes included almost 50 crashes. It remains a little unclear which factors influence their choice of such an enormous amount of crashes in such a short period. Researchers emphasize these drops as such, with a fall of more than $15 \%$ and a duration of fewer than three weeks. Nevertheless, regarding this classification, we are going to emphasize the most relevant, where the complexity of the index started to decrease and whose initial deviation from regular behavior was noticeable in advance. Nowadays some people proclaim Bitcoin as a "digital gold". Gold as a material has served for jewelry and art as well as electronic or medical components. Limited supply and current acceptance of Bitcoin as a "digital gold" may erect it to the same level as gold. While some people back up Bitcoin's advantage, demonstrating its similarities with those of gold and silver [71], others argue that it is the new digital coin [72] due to its high volatility and unclear future development. However, researchers find its potential benefits during extreme market periods and provide a set of stylized factors that claim to be successful long-short strategies that generate sizable and statistically significant excess returns [73]. Despite volatile swings and many crit- 
ics, Bitcoin has emerged and attracted much more confidence. These studies $[74,75]$ consider that measures of financial and macroeconomic activity can be drivers of Bitcoin returns. Reviewing papers of the researches above, the experience of others and our own [76-85], we have revised our classification of such leaps and falls, relying on Bitcoin time series during the entire period (01.01.201121.01.2021) of verifiable fixed daily values of the Bitcoin price (BTC) (https://finance.yahoo.com/cryptocurrencies). We emphasize that

- crashes are short and time-localized drops that last approximately two weeks, with the weighty losing of price each day. Their volatility is high. In percentage term, their decline exceeds 30 percent, and normalized returns proceed $\pm 3 \sigma$ or near to it;

- critical events are those falls that, during their existence, have not had such massive changes in price as crashes.

Relying on these considerations, we emphasize 29 periods on Bitcoin time series, relying on normalized returns and volatility, where returns are calculated as

$$
G(t)=\ln x(t+\Delta t)-\ln x(t) \cong[x(t+\Delta t)-x(t)] / x(t)
$$

and normalized (standardized) returns as

$$
g(t) \cong[G(t)-\langle G\rangle] / \sigma,
$$

where $\sigma \equiv \sqrt{\left\langle G^{2}\right\rangle-\langle G\rangle^{2}}$ is the standard deviation of $G$, $\Delta t$ is time lag (in our case $\Delta t=1$ ), and $\langle\ldots\rangle$ denotes the average over the time period under study and volatility as

$$
V_{T}(t)=\frac{1}{n} \sum_{t^{\prime}=t}^{t+n-1}\left|g\left(t^{\prime}\right)\right|
$$

From the mentioned stylized facts on BTC dynamics, it was noticed how considerably it started to change near 2014. To gain a deeper understanding of its existence in the starting period, we divided the BTC time series into two periods: (01.01.2011-31.08.2016) and (01.09.201621.01.2021). More detailed information about crises, crashes, and their classification under these definitions is given in table 1 and table 2.

Therefore, according to our classification crisis periods with numbers $(1,2,4-6,8,9,13,18,23-25,27,29)$ are crashes, all the rest - critical events.

Figure 2 confirms the importance of dividing the BTC time series in order to observe its dynamics in more detail. However, as it can be seen, we could separate time series in much deeper time scales.

In figure 3 output Bitcoin time series for the first and the second periods, their normalized returns $g(t)$, and volatility $V_{T}(t)$ calculated for the window of size 100 are presented.

From figure 3 we can see that during periods of crashes and critical events normalized returns $g$ increases considerably in some cases beyond the limits $\pm 3 \sigma$. This indicates deviation from the normal law of distribution, the presence of the "heavy tails" in the distribution $g$, which are characteristics of abnormal phenomena in the market. At the same time volatility also grows. Such qualities are the foundation of our classification for crashes, as it has been mentioned already. All the rest events are critical. These characteristics serve as indicators of crashes and critical events as they react only at the moment of the abovementioned phenomena and do not allow identifying the corresponding abnormal phenomena in advance. In contrast, most of the indicators described below will respond to critical changes and crashes in advance. It enables them to be used as indicators - precursors of such phenomena.

Calculations were carried out within the framework of the algorithm of a rolling (sliding, moving) window. For this purpose, the part of the time series (window), for which there were calculated measures of complexity, was selected, then the window was displaced along with the time series in a predefined value, and the procedure repeated until all the studied series had exhausted. Further, comparing the dynamics of the actual time series and the corresponding measures of complexity, we can judge the characteristic changes in the dynamics of the behavior of complexity with changes in the cryptocurrency. If this or that measure of complexity behaves in a definite way for all periods of crashes, for example, decreases or increases during the pre-crashes or pre-critical period, then it can serve as their indicator or precursor.

Calculations of measures of complexity were carried out both for the entire time series, and for a fragment of the time series localizing some of the emphasized crashes and critical events. In the latter case, fragments of time series of the same length with fixed points of the onset of crashes or critical events were selected and the results of calculations of complexity measures were compared to verify the universality of the indicators. Following some described below procedures such time localization as, example, of length 100 or 200, either won 't make any sense, or won't be possible as some of them are sensitive to time localization, or require a longer length of the time series as it is required by the procedure for better accuracy of further calculations

\section{Informational measures of complexity}

Complexity is a multifaceted concept, related to the degree of organization of systems. Patterns of complex organization and behavior are identified in all kinds of systems in nature and technology. Essential for the characterization of complexity is its quantification, the introduction of complexity measures, or descriptors [86].

We may speak of the complexity of a structure, meaning the amount of information (number of bits) of the structure; this is the minimum space we need to store enough information about the structure that allows us its reconstruction. We may also speak of the algorithmic complexity of a certain task: this is the minimum time (or other computational resources) needed to carry out this task on a computer. And we may also speak of the communication complexity of tasks involving more than one processor: this is the number of bits that have to be transmitted in solving this task [87-89]. 
Table 1: List of Bitcoin major crashes and critical events since June 2011 till July 2016

\begin{tabular}{ccccccc}
\hline № & Name & $\begin{array}{c}\text { Days in } \\
\text { correction }\end{array}$ & $\begin{array}{c}\text { Bitcoin High } \\
\text { Price, } \$\end{array}$ & $\begin{array}{c}\text { Bitcoin Low } \\
\text { Price, } \$\end{array}$ & $\begin{array}{c}\text { Decline, } \\
\%\end{array}$ & $\begin{array}{c}\text { Decline, } \\
\text { ( }\end{array}$ \\
\hline 1 & $07.06 .2011-10.06 .2011$ & 3 & 29.60 & 14.65 & 50 & 14.95 \\
2 & $11.10 .2011-18.10 .2011$ & 7 & 4.15 & 2.27 & 45 & 1.88 \\
3 & $15.01 .2012-16.02 .2012$ & 32 & 7.00 & 4.27 & 39 & 2.73 \\
4 & $15.08 .2012-18.08 .2012$ & 3 & 13.50 & 8.00 & 40 & 5.50 \\
5 & $08.04 .2013-15.04 .2013$ & 7 & 230.00 & 68.36 & 70 & 161.64 \\
6 & $28.04 .2013-02.05 .2013$ & 4 & 144.00 & 98.09 & 32 & 45.91 \\
7 & $19.06 .2013-04.07 .2013$ & 15 & 111.29 & 68.50 & 38 & 42.79 \\
8 & $04.12 .2013-07.12 .2013$ & 3 & 1237.66 & 697.02 & 44 & 540.64 \\
9 & $05.02 .2014-21.02 .2014$ & 16 & 904.52 & 111.55 & 88 & 792.97 \\
10 & $24.03 .2014-09.04 .2014$ & 17 & 567.56 & 384.63 & 32 & 182.93 \\
11 & $09.08 .2014-17.08 .2014$ & 8 & 592.06 & 462.18 & 22 & 129.88 \\
12 & $22.09 .2014-04.10 .2014$ & 12 & 436.86 & 322.86 & 26 & 114.00 \\
13 & $12.01 .2015-14.01 .2015$ & 2 & 269.33 & 164.92 & 39 & 104.41 \\
14 & $27.07 .2015-23.08 .2015$ & 27 & 293.70 & 211.43 & 28 & 82.27 \\
15 & $09.11 .2015-11.11 .2015$ & 2 & 380.22 & 304.71 & 28 & 75.51 \\
16 & $18.06 .2016-21.06 .2016$ & 3 & 761.04 & 590.56 & 22 & 170.48 \\
17 & $29.07 .2016-01.08 .2016$ & 3 & 654.74 & 513.43 & 24 & 141.31
\end{tabular}

Table 2: List of Bitcoin major crashes and critical events since January 2017 till March 2020

\begin{tabular}{ccccccc}
\hline № & Name & $\begin{array}{c}\text { Days in } \\
\text { correction }\end{array}$ & $\begin{array}{c}\text { Bitcoin High } \\
\text { Price, } \$\end{array}$ & $\begin{array}{c}\text { Bitcoin Low } \\
\text { Price, } \$\end{array}$ & $\begin{array}{c}\text { Decline, } \\
\%\end{array}$ & $\begin{array}{c}\text { Decline, } \\
\$\end{array}$ \\
\hline 18 & $04.01 .2017-11.01 .2017$ & 7 & 1135.41 & 785.42 & 30 & 349.99 \\
19 & $15.03 .2017-18.03 .2017$ & 3 & 1253.43 & 971.38 & 23 & 282.05 \\
20 & $10.06 .2017-15.07 .2017$ & 35 & 2973.44 & 1914.09 & 36 & 1059.35 \\
21 & $31.08 .2017-13.09 .2017$ & 13 & 4921.85 & 3243.08 & 34 & 1678.77 \\
22 & $08.11 .2017-12.11 .2017$ & 4 & 7444.36 & 5878.13 & 21 & 1566.23 \\
23 & $16.12 .2017-30.12 .2017$ & 14 & 19345.49 & 12531.52 & 35 & 6813.97 \\
24 & $06.01 .2018-05.02 .2018$ & 30 & 17172.30 & 6937.08 & 60 & 10235.22 \\
25 & $04.03 .2018-05.04 .2018$ & 33 & 11504.42 & 6634.86 & 42 & 4869.56 \\
26 & $04.05 .2018-27.05 .2018$ & 23 & 9845.90 & 7118.88 & 28 & 2727.02 \\
27 & $18.11 .2018-15.12 .2018$ & 27 & 5615.26 & 3232.51 & 42 & 2382.75 \\
28 & $12.07 .2019-16.07 .2019$ & 4 & 11797.37 & 9423.44 & 20 & 2373.93 \\
29 & $06.03 .2020-16.03 .2020$ & 10 & 9122.55 & 5014.48 & 45 & 4108.07
\end{tabular}

Historically, the first attempt to quantify complexity was based on Shannon's information theory [90] and Kolmogorov complexity [91].

\subsection{Lempel-Ziv complexity}

Lempel-Ziv complexity (LZC) is a classical measure that, for ergodic sources, relates the concepts of complexity (in the Kolmogorov-Chaitin sense), and entropy rate [92, 93]. For an ergodic dynamical process, the amount of new information gained per unit of time (entropy rate) can be estimated by measuring the capacity of this source to generate new patterns (LZC). Because of the simplicity of the LZC method, the entropy rate can be estimated from a single discrete sequence of measurements with a low computational cost [94].

In this paper, we show that the LZC measure can be just such a measure of complexity, which is an early pre- cursor of crisis phenomena in the cryptocurrency market $[2,79,95,96]$.

Historically, the first LZC measure system studies for financial time series were conducted by S Da Silva et al. [96-99]. They considered the deviation of LZC from that value for a random time series as a measure of actual market efficiency in absolute [95, 96, 98, 99] or relative [97] terms. Using this approach authors were able to detect decreases in efficiency rates of the major stocks listed on the Sao Paulo Stock Exchange in the aftermath of the 2008 financial crisis [98]. In [100], authors have surveyed the principal applications of algorithmic (Kolmogorov) complexity to the problem of financial price motions and showed the relevance of the algorithmic framework to structure tracking in finance. Some empirical results are also provided to illustrate the power of the proposed estimators to take into account patterns in stock returns. In paper [101] was proposed a generic methodology to esti- 


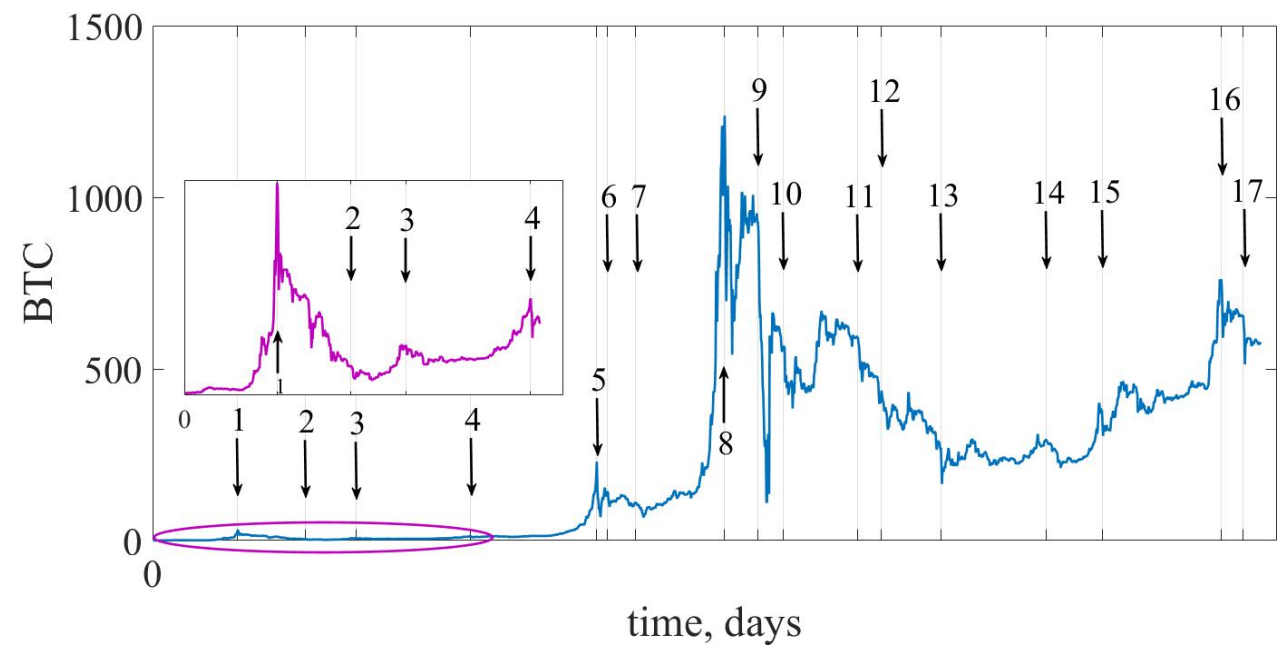

Figure 2: The dynamics of the daily values of the BTC price. The inset shows 1-4 crisis periods presented in table 1.

mate the Kolmogorov complexity of financial returns. Examples are given with simulated data that illustrate the advantages of our algorithmic method: among others, some regularities that cannot be detected with statistical methods can be revealed by compression tools. Applying compression algorithms to daily returns of the Dow Jones Industrial Average (DJIA), the authors concluded on an extremely high Kolmogorov complexity and by doing so, proposed another empirical observation supporting the impossibility to outperform the market. The structural complexity of time series describing returns on New York's and Warsaw's stock exchanges was studied using two estimates of the Shannon entropy rate based on the Lepel-Ziv and Context Tree Weighting algorithms [102]. Such structural complexity of the time series can be used as a measure of the internal (modelless) predictability of the main pricing processes and testing the hypothesis of an efficient market. Somewhat surprisingly, the results of [103], in which the authors computed the LZC from two composite stock indices, the Shanghai stock exchange composite index (SSE) and the DJIA, for both low-frequency (daily) and high-frequency (minute-to-minute) stock index data. The calculation results indicate that that the US market is basically fully random and consistent with the efficient market hypothesis (EMH), irrespective of whether low- or high-frequency stock index data are used. The Chinese market is also largely consistent with the EMH when lowfrequency data are used. However, a completely different picture emerges when the high-frequency stock index data are used. H. Cao and Y. Li [104] presents a novel method for measuring the complexity of a time series by unraveling a chaotic attractor modeled on complex networks. The complexity index, which can potentially be exploited for prediction, has a similar meaning to the LZC and is an appropriate measure of a series' complexity. The proposed method is used to research the complexity of the world's major capital markets. The almost absent sensitivity of the LZC to fluctuations in the time series indicates most likely errors in the calculation algorithm during the transformation of the time series. The complexity-entropy causality plane is employed in order to explore disorder and complexity in the space of cryptocurrencies [104]. They are found to exist in distinct planar locations in the representation space, ranging from structured to stochastic-like behavior.

A brief analysis of the problem indicates that so far, the Lempel-Ziv informational measure of the complexity has not been used to study the stability and behavior of the cryptocurrency market in a crisis. In this section, we use the Lempel-Ziv complexity measure to study the cryptocurrency market. Using the example of the most capitalized cryptocurrency - Bitcoin - we demonstrate the ability to identify the dynamics of varying complexity. Particularly relevant is the identification of the characteristic behavior of Bitcoin during the crisis phases of market behavior. By observing the dynamics of the Lempel-Ziv measure, precursors of crisis phenomena can be constructed [105].

\subsubsection{The concept of Kolmogorov complexity}

Let us begin with the well-known degree of complexity proposed by Kolmogorov [106]. The concept of Kolmogorov complexity (or, as they say, algorithmic entropy) emerged in the 1960s at the intersection of algorithm theory, information theory, and probability theory. A. Kolmogorov's idea was to measure the amount of information contained in individual finite objects (rather than random variables, as in the Shannon theory of information). It turned out to be possible (though only to a limited extent). A. Kolmogorov proposed to measure the amount of information in finite objects using algorithm theory, defining the complexity of an object as the minimum length of the program that generates that object. This definition is the basis of algorithmic information theory as well as algorithmic probability theory: an object is considered random if its complexity is close to maximum.

What is the Kolmogorov complexity and how to measure it? In practice, we often encounter programs that compress files (to save space in the archive). The most 


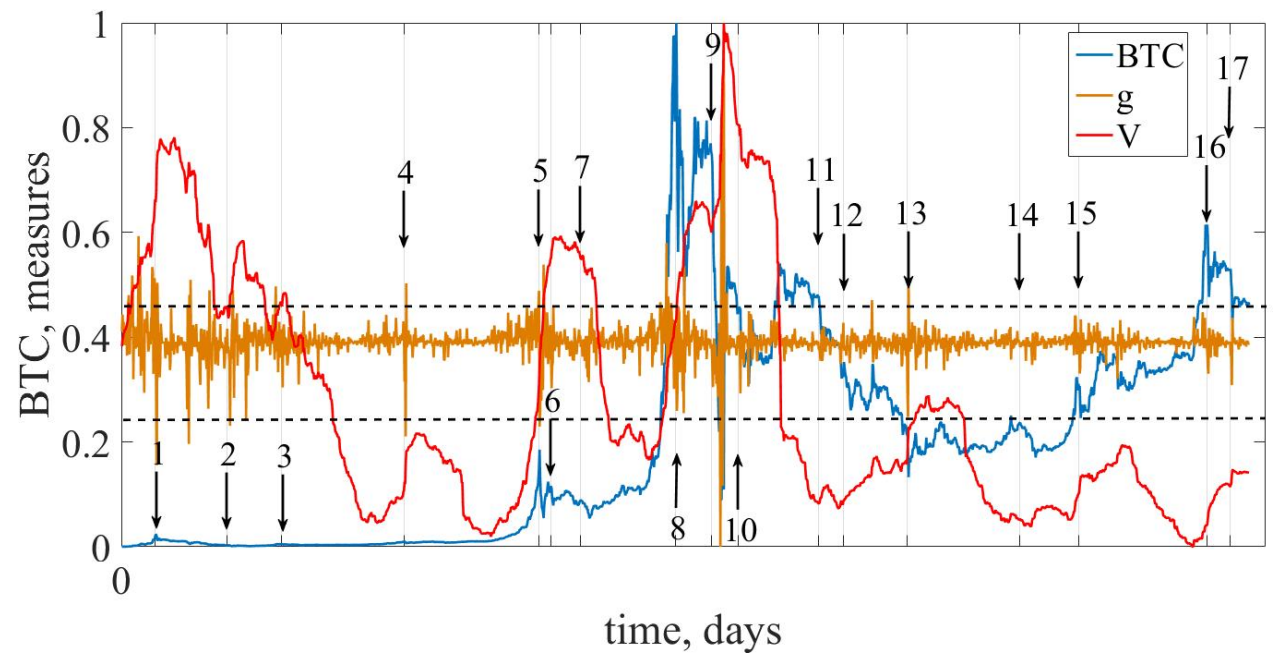

(a)

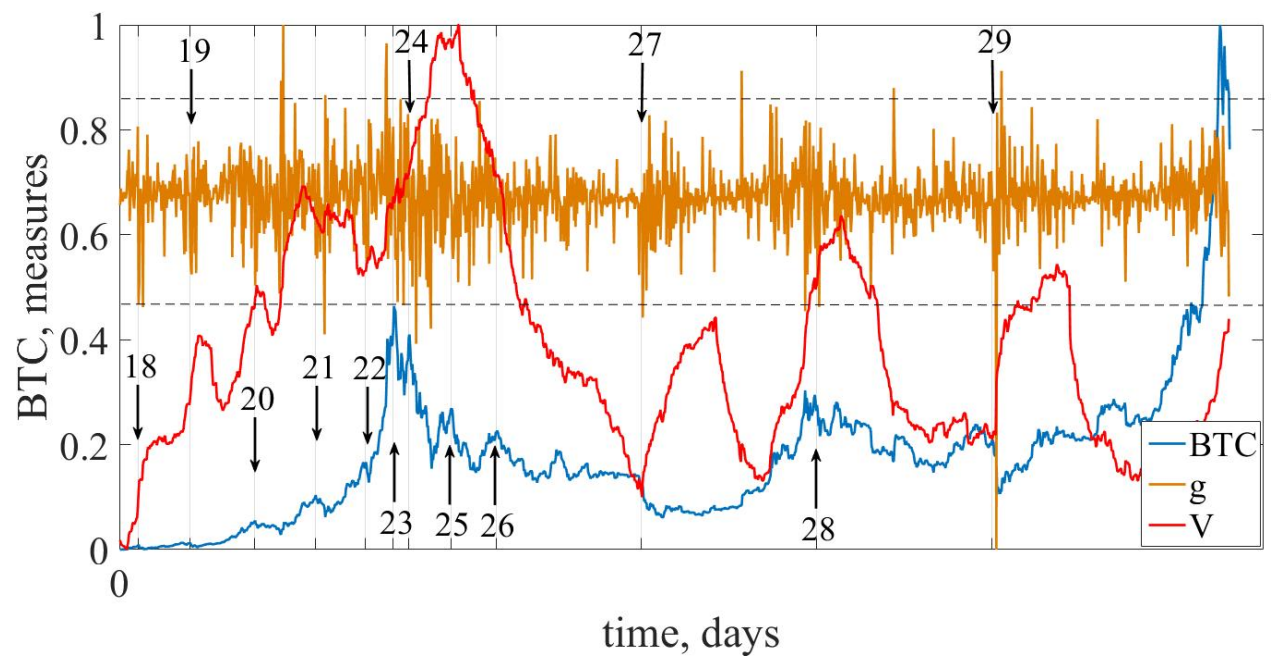

(b)

Figure 3: The standardized dynamics, returns $g(t)$, and volatility $V_{T}(t)$ of BTC/USD daily values for first (a) and second (b) periods. Horizontal dotted lines indicate the $\pm 3 \sigma$ borders. The arrows indicate the beginning of one of the crashes or the critical events

common are called zip, gzip, compress, rar, arj, and others. Applying such a program to some file (with text, data, program), we get its compressed version (which is usually shorter than the original file). After that, you can restore the original file using the paired program "decompressor". Therefore, approximately, the Kolmogorov complexity of a file can be described as the length of its compressed version. Thus, a file that has a regular structure and is well compressed has a small Kolmogorov complexity (compared to its length). On the contrary, a badly compressed file has a complexity close to its length.

Suppose we have a fixed method of description (decompressor) $D$. For this word $x$, we consider all its descriptions, i.e., all words $y$ for which $D(y)$ it is defined and equal to $x$. The length of the shortest of them is called the Kolmogorov complexity of the word $x$ in this way of description $D$ :

$$
K S_{D}(x)=\min \{l(y) \mid D(y)=x\}
$$

where $l(y)$ denotes the length of the word. The index $D$ emphasizes that the definition depends on the chosen method $D$. It can be shown that there are optimal methods of description. The better the description method, the shorter it is. Therefore, it is natural to make the following definition: the method $D_{1}$ is no worse than the method $D_{2}$ if

$$
K S_{D_{1}} \leq K S_{D_{2}}(x)+c
$$

for some $c$ and all $x$.

Thus, according to Kolmogorov, the complexity of an object (for example, the text is a sequence of characters) is the length of the minimum program that outputs the text, and entropy is the complexity that is divided by the length of the text. Unfortunately, this definition is purely spec- 
ulative. There is no reliable way of identifying this program uniquely, but there are algorithms that are actually just trying to calculate the Kolmogorov complexity of text and entropy.

A universal (in the sense of applicability to different language systems) measure of the complexity of the finite character sequence was suggested by Lempel and $\mathrm{Ziv}$ [100]. As part of their approach, the complexity of a sequence is estimated by the number of steps in the process that gives rise to it.

Acceptable (editorial) operations are: a) character generation (required at least for the synthesis of alphabet elements) and b) copying the "finished" fragment from the prehistory (i.e. from the already synthesized part of the text).

Let be $\Sigma$ a complete alphabet, $S$ - text (a sequence of characters) composed of elements $\Sigma ; S[i]-i^{\text {th }}$ text symbol; $S[i: j]$ - a snippet of text from the $i^{\text {th }}$ to $j^{\text {th }}$ character inclusive $(i<j) ; N=|S|-$ length of text $S$. Then the sequence synthesis scheme can be represented as a concatenation

$$
\begin{array}{r}
H(S)=S\left[1: i_{1}\right] S\left[i_{1}+1: i_{2}\right] \ldots \\
\ldots S\left[i_{k-1}+1: i_{k}\right] \ldots S\left[i_{m-1}+1: N\right],
\end{array}
$$

where $S\left[i_{k-1}+1: i_{k}\right]$ is the fragment $S$ generated at the $k^{\text {th }}$ step, and $m=m_{H}(S)$ is the number of process steps. Of all the schemes of generation is chosen the minimum number of steps. Thus, the Lempel-Ziv complexity of the sequence $S$ is

$$
c_{L Z}(S)=\min _{H}\left\{m_{H}(S)\right\} .
$$

The minimum number of steps is provided by the choice to copy at each step the longest prototype from the prehistory. If you mark by the position number $j(k)$ from which the copying begins in step $k$ the length of the copy fragment

$$
\begin{array}{r}
l_{j(k)}=i_{k}-i_{k-1}-1=\max _{j \leq i_{k-1}}\left\{l_{j}: S\left[i_{k-1}+1: i_{k-1}+l_{j}\right]=\right. \\
\left.=S\left[j: j+l_{j}-1\right]\right\}
\end{array}
$$

and the $k^{\text {th }}$ component of these complex decomposition can be written in the form

$$
S\left[i_{k-1}+1: i_{k}\right]= \begin{cases}S\left[j(k): j(k)+l_{j(k)}-1\right] & \text { if } j(k) \neq 0, \\ S\left[i_{k-1}+1\right] & \text { if } j(k)=0 .\end{cases}
$$

The case $j(k)=0$ corresponds to a situation where a symbol is in the position $i_{k-1}+1$ that has not been encountered previously. In doing so, we use a character generation operation.

Complex text analysis can be performed in two regimes - segmentation and fragmentation. The first regime is discussed above. It gives an integrated view of the structure of the sequence as a whole and reduces it to disjoint but interconnected segments (without spaces). The other regime is to search for individual fragments characterized by an abnormally low complexity which means that they characterized by a sufficiently high degree of structure. Such fragments are detected by calculating local complexity within variable-length windows that slide along a sequence. Curves of change of local complexity along a sequence are called complex profiles. A set of profiles for different window sizes reveals the boundaries of anomalous fragments and their relationship.

We will find the LZC complexity for the time series, which is, for example, the daily values of the cryptocurrency price $x(t)$. To investigate the dynamics of LZC and compare it with cryptocurrency prices, we will find this measure of complexity for a fixed length (window) contract. To do this, we calculate the logarithmic returns accordingly to equation (1) and turn them into a sequence of bits.

You can specify the number of states that are differentiated (calculus system). Yes, for two different states we have 0,1 , for three $-0,1,2$, etc. In the case of three states, unlike the binary coding system, a certain threshold $\sigma$ is set and the states $g$ are coded as follows [96, 98, 99]:

$$
g= \begin{cases}0 & \text { if } g<-\sigma, \\ 1 & \text { if }-b \leq g \leq b, \\ 2 & \text { if } g>b .\end{cases}
$$

The algorithm performs two operations: (1) adds a new bit to an already existing sequence; (2) copies the already formed sequence. Algorithmic complexity is the number of such operations required to form a given sequence.

For a random sequence of lengths $n$, the algorithmic complexity is calculated by expression $L Z C_{r}=n / \log n$. Then, the relative algorithmic complexity is the ratio of the obtained complexity to the complexity of the random sequence $L Z C=L Z C / L Z C_{r}$.

Obviously, the classical indicators of algorithmic complexity are unacceptable and lead to erroneous conclusions. To overcome such difficulties, multiscale methods are used.

The idea of this group of methods includes two consecutive procedures: 1) coarse-graining ("granulation") of the initial time series - the averaging of data on nonintersecting segments, the size of which (the window of averaging) increased by one when switching to the next largest scale; 2) computing at each of the scales a definite (still mono scale) complexity indicator. The process of "rough splitting" consists in the averaging of series sequences in a series of non-intersecting windows, and the size of which - increases in the transition from scale to scale [107]. Each element of the "granular" time series follows the expression:

$$
y_{j}^{\tau}=\frac{1}{\tau} \sum_{i=(j-1) \tau+1}^{j \tau} g(i), \quad \text { for } 1 \leq j \leq N / \tau,
$$

with corresponding scale factor $\tau$. The length of each "granular" row depends on the length of the window and is even $N / \tau$. For a scale of 1 , the "granular" series is exactly identical to the original one.

The coarse graining procedure for scales 2 and 3 is shown in figure 4. 


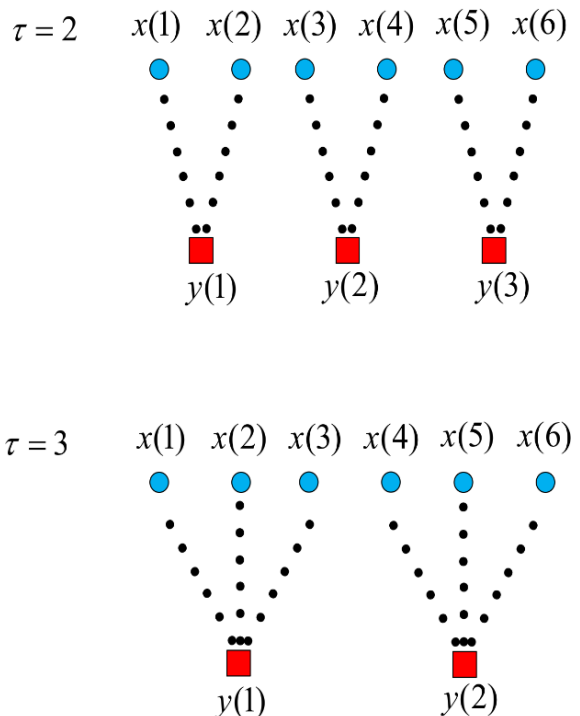

(b)

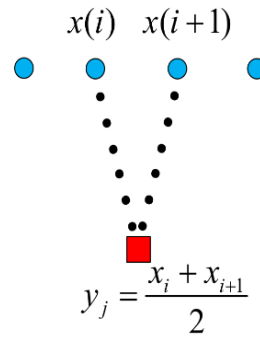

(a)

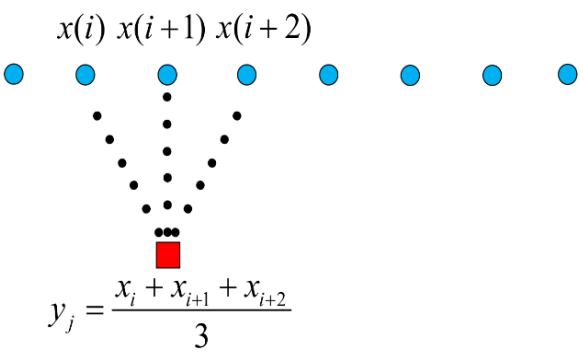

Figure 4: Coarse-graining procedure diagram: (a) scale factor $\tau=2$; (b) scale factor $\tau=3$

To find the LZC measure of the time series, the rolling time windows were considered; the index for every window was calculated, and then the average was obtained.

Obviously, the crisis in the cryptocurrency market responds to noticeable fluctuations in standardized returns. Therefore, it is logical to choose $\sigma$ as the value for the threshold value $b$.

Figure 5 shows the dependence of the LZC on the scale. The absence of LZC fluctuations at scales exceeding 40 allows us to confine ourselves to this magnitude of the scale when calculating the multiscale measure.

Calculations of measures of complexity were carried for the two periods of BTC. Figure 6 presents the results of calculations of mono- $\left(L Z C_{m 1}\right)$ and multi- $\left(L Z C_{m 40}\right)$ scaling LZC measures. The calculations were performed for a rolling window of 100 days and an increment of 1 day.

The data in figure 6 indicate that the LZC measure is noticeably reduced both in the case of mono-scale $\left(m_{1}\right)$ and averaged over the scales from 1 to $40\left(m_{40}\right)$ for all 29 crashes and critical events in the immediate vicinity of the crisis point.

As the results of calculations showed, the choice of the size of a rolling window is important: in the case of large windows, points of crises of different times can fall into the window, distorting the influence of each of the crises. When choosing small windows, the results fluctuate greatly, which makes it difficult to determine the actual point of the crisis. The used window length of 100 days turned out to be optimal for the separation of crises and fixing the LZC measure as an indicator.

Since the LZC measure begins to decrease even before the actual crisis point, it can be called an indicatorprecursor of crisis phenomena in the cryptocurrency market.

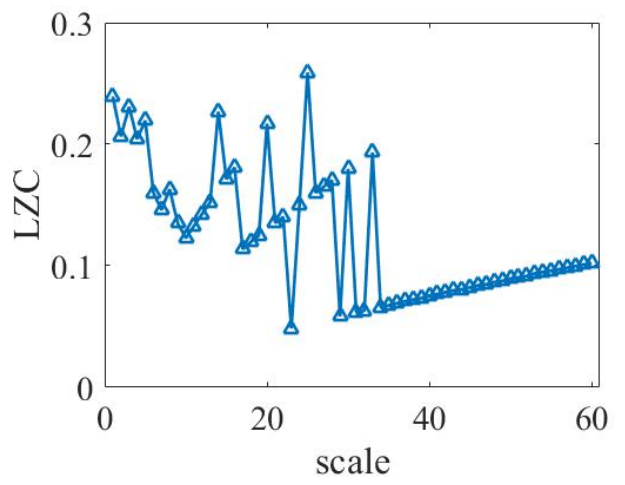

(a)

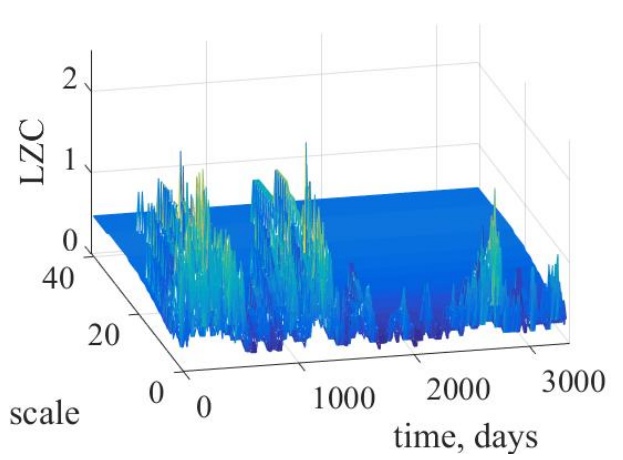

(b)

Figure 5: Scale-dependent LZC (a) and its version with the rolling window approach (b)

\subsection{Entropy as a measure of complexity}

Nowadays, the most important quantity that allows us to parameterize complexity in deterministic or random processes is entropy. Originally, it was introduced by R. Clau- 


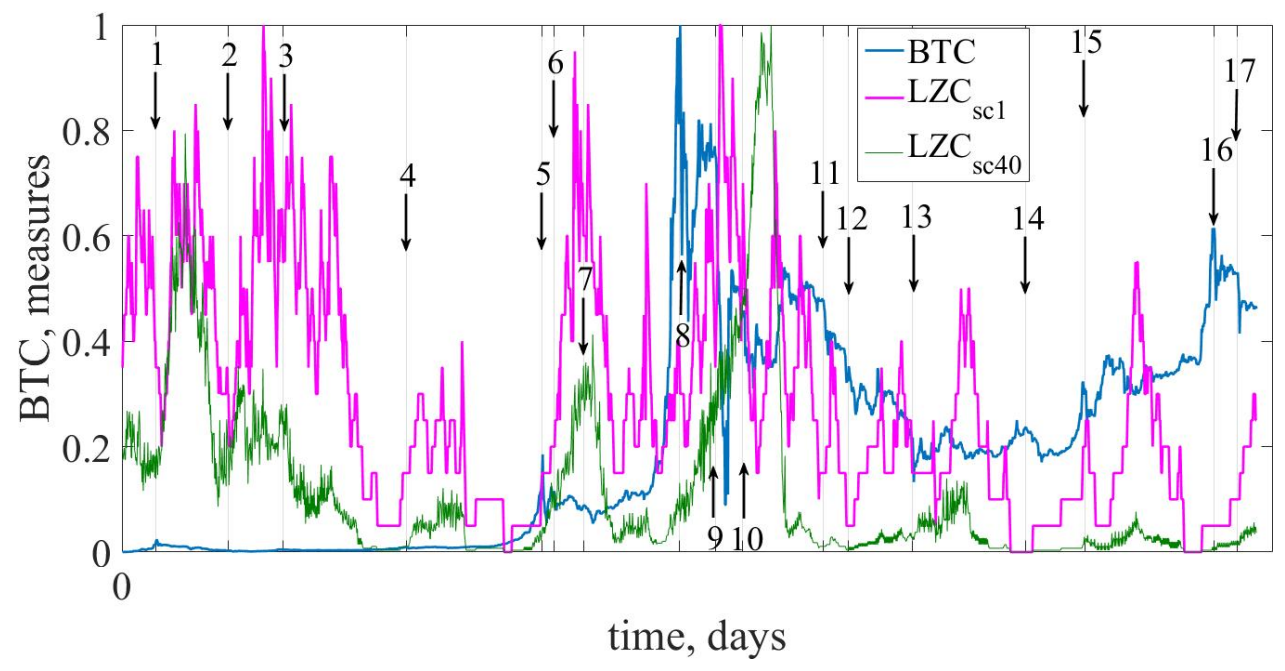

(a)

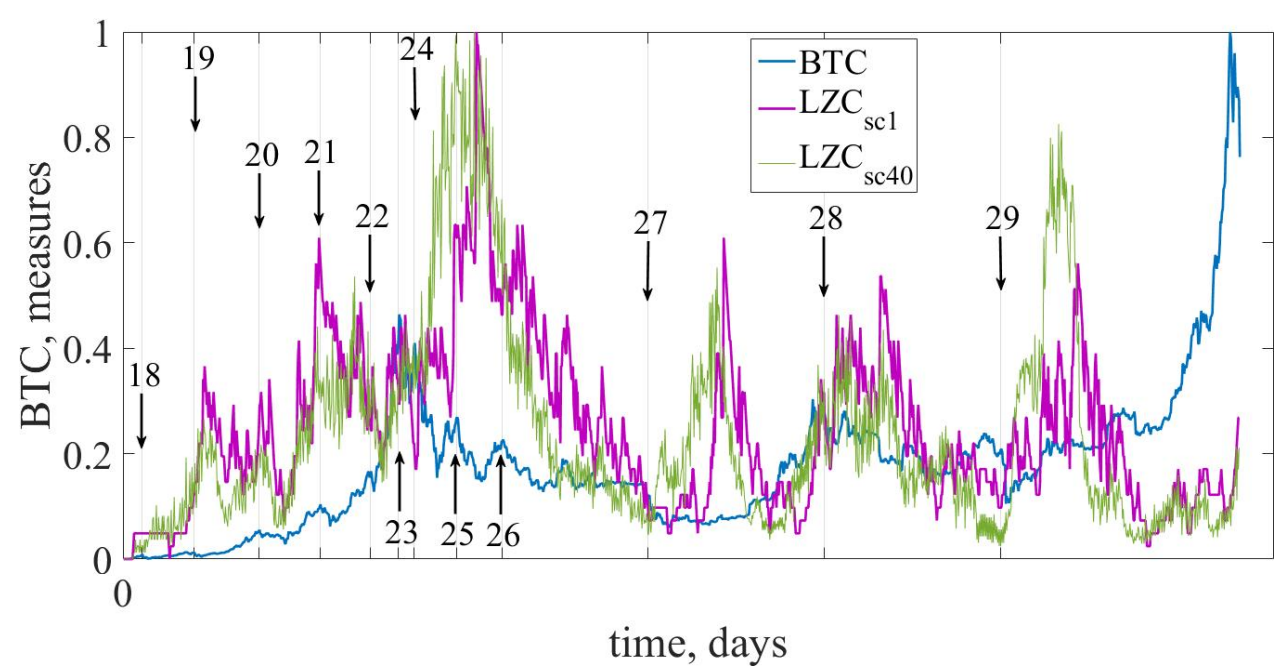

(b)

Figure 6: Comparative dynamics of BTC price fluctuations and mono- and multi-scaling LZC measures for first (a) and second (b) periods of the entire time series of Bitcoin

sius [108], in the context of classical thermodynamics, where according to his definition, entropy tends to increase within an isolated system, forming the generalized second law of thermodynamics. Then, the definition of entropy was extended by Boltzmann and Gibbs [109, 110], linking it to molecular disorder and chaos to make it suitable for statistical mechanics, where they combined the notion of entropy and probability [111].

After the fundamental paper of Shannon [90] in the context of information theory, where entropy denoted the average amount of information contained in the message, its notion was significantly redefined. After this, it has been evolved along with different ways and successful enough used for the research of economic systems [112115].

A huge amount of different methods, as an example, from the theory of complexity, the purpose of which is to quantify the degree of complexity of systems obtained from various sources of nature, can be applied in our study. Such applications have been studied intensively for an economic behavior system.

The existence of patterns within the series is the core in the definition of randomness, so it is appropriate to establish such methods that will be based on the different patterns and their repetition [116]. In this regard, Pincus described the methodology Approximate entropy (ApEn) [117] to gain more detail analysis of relatively short and noisy time series, particularly, of clinical and psychological. Its development was motivated by the length constraints of biological data. Since then it has been used in different fields such as psychology [118], psychiatry [119], and finance [120-124]. Pincus co-authored with Kalman [124], considering both empirical data and models, including composite indices, individual stock prices, the random-walk hypothesis, Black-Sholes, and fractional Brownian motion models to demonstrate the benefits of 
ApEn applied to the classical econometric modeling apparatus. This research the usefulness of ApEn on the example of three major events of the stock market crash in the US, Japan, and India. During the major crashes, there is significant evidence of a decline of ApEn during and pre-crash periods. Based on the presented results, their research concludes that ApEn can serve as a base for a good trading system. Duan and Stanley [125] showed that it is possible to effectively distinguish the real-world financial time series from random-walk processes by examining changing patterns of volatility, approximate entropy, and the Hurst exponent. The empirical results prove that financial time series are predictable to some extent and ApEn is a good indicator to characterize the predictable degree of financial time series. Alfonso Delgado-Bonal [126] gives evidence of the usefulness of ApEn. The researcher quantifies the existence of patterns in evolving data series. In general, his results present that degree of predictability increases in times of crisis.

Permutation entropy (PEn), according to the previous approach, is a complexity measure that is related to the original Shannon entropy (ShEn) that applied to the distribution of ordinal patterns in time series. Such a tool was proposed by Bandt and Pompe [127], which is characterized by its simplicity, computational speed that does not require some prior knowledge about the system, strongly describes nonlinear chaotic regimes. Also, it is characterized by its robustness to noise $[128,129]$ and invariance to nonlinear monotonous transformations [130]. The combination of entropy and symbolic dynamics turned out to be fruitful for analyzing the disorder for the time series of any nature without losing their temporal information.

As an example, Henry and Judge [131] applied PEn to the Dow Jones Industrial Average (DJIA) to extract information from this complex economic system. The result demonstrates the ability of the PEn method to detect the degree of disorder and uncertainty for the specific time that is explored. Higor et al. [132] applied PEn and statistical complexity over sliding time-window of daily closing price log-returns to quantify the dynamic efficiency of more than four hundred cryptocurrencies. Authors address to the efficient market hypothesis when the values of two statistical measures within a time-window cannot be distinguished from those obtained by chance. They found that $37 \%$ of the cryptocurrencies in their study stayed efficient over $80 \%$ of the time, whereas $20 \%$ were informationally inefficient in less than $20 \%$ of the time. Moreover, the market capitalization was not correlated with their efficiency. Performed analysis of information efficiency over time reveals that different currencies with similar temporal patterns formed four clusters, and it was seen that more young currencies tend to follow the trend of the most leading currencies. Sensoy [133] compared the time-varying weak-form efficiency of Bitcoin prices in terms of US dollars (BTC/USD) and euro (BTC/EUR) at a high-frequency level by PEn. He noticed that BTC/USD and BTCEUR have become more informationally useful since the beginning of 2016, namely Bitcoin in the same period. Researcher also found that with higher frequency in the Bitcoin market, we had lower price efficiency. Moreover, cryptocurrency liquidity (volatility) had a significant positive (negative) effect on the informational efficiency of its price.

Also, Ayşe Metin Karakaş dedicated [134] both to Bitcoin and Ethereum. Here, the concept of entropy was applied for characterizing the nonlinear properties of the cryptocurrencies. For her goal, Shannon, Tsallis, Rényi, and Approximate entropies were estimated. From her empirical results, it was obtained that all entropies were positive. Of great interest was the results of ApEn which demonstrated larger value for Ethereum than for Bitcoin. In this case, it concluded that Ethereum had higher volatility.

Pele [135] investigated the ability of several econometrical models to forecast value at risk for a sample of daily time series of cryptocurrency returns. Using highfrequency data for Bitcoin, they estimated the entropy of the intraday distribution of log-returns through the symbolic time series analysis STSA, producing low-resolution data from high-resolution data. Their results showed that entropy had strong explanatory power for the quantiles of the distribution of the daily returns. They confirmed the hypothesis that there was a strong correlation between the daily logarithmic price of Bitcoin and the entropy of intraday returns based on Christoffersen's tests for Value at Risk (VaR) backtesting, they concluded that the VaR forecast built upon the entropy of intraday returns was the best, compared to the forecasts provided by the classical GARCH models.

\subsubsection{Time delay method}

The state of the system can be described by the set of variables. Its observational state can be expressed through a $d$-dimensional vector or matrix, where each of its components refers to a single variable that represents a property of the system. After a while, the variables change, resulting in different system states.

Usually, not all relevant variables can be captured from our observations. Often, only a single variable may be observed. Thakens' theorem [136] that was mentioned in previous sections ensures that it's possible to reconstruct the topological structure of the trajectory formed by the state vectors as the data collected for this single variable contains information about the dynamics of the whole system.

For an approximate reconstruction of the original dynamics of the observed system, we project the time series onto a Reconstructed Phase Space $[130,137,138]$ with the commonly used time delay method [130] which relied on the embedding dimension and time delay.

The embedding dimension is being the dimensionality of the reconstructed system (corresponds to the number of relevant variables that may differ from one system to another. The time delay parameter specifies the temporal components of the vector components. As an example, in recurrence analysis that will be described in section 6 , Webber and Zbilut [139] recommend setting the embedding dimension between 10 and 20. Regarding the analysis of financial systems, values between 1 and 20 for the 
embedding dimension are considered to be reasonable as well as the time delay.

\subsubsection{Shannon entropy}

The general approach can be described as follows. Formally, we represent the underlying dynamic state of the system in probability distribution form $P$ and then the Shannon entropy $S$ with an arbitrary base (i.e. 2, e, 10) is defined as:

$$
S[P]=-\sum_{i=1}^{N} p_{i} \log p_{i}
$$

Here, in equation $4, p_{i}$ represents the probability that price $i$ occurs in the sample's distribution of the Bitcoin time series, and $N$ is the total amount of data in our system. Dealing with continuous probability distributions with a density function $f(x)$, we can define the entropy as:

$$
H(f)=-\int_{-\infty}^{+\infty} f(x) \log f(x) d x .
$$

According to the approach, the negative log increases with rarer events due to the information that is encoded in them (i.e., they surprise when they occur). Thus, when all $p_{i}$ have the same value, i.e. where all values are equally probable, and $S[P]$ reaches its minimum for more structured time series (events that are more certain). Equation 5 is obeyed to the same rules as 4 . In figure 7 are the empirical results for Shannon entropy and Bitcoin time series.

It can be seen from the figure that Shannon's entropy is rapidly increasing at the very moment of the crisis itself and is an excellent indicator of crisis phenomena.

\subsubsection{Approximate entropy}

To gain more detail analysis of the complex financial systems, it is known other entropy methods have become known, particularly, ApEn developed by Pincus [117] for measuring regularity in a time series.

When calculating it, given $N$ data points $\{x(i) \mid i=1, \ldots, N\}$ are transformed into subvectors $\overrightarrow{\mathrm{X}}(i) \in \mathfrak{R}^{d_{E}}$, where each of those subvectors has $\left[x(i), x(i+1), \ldots, x\left(i+d_{E}-1\right)\right]$ for each $i, 1 \leq i \leq N-m+1$. Correspondingly, for further estnimations, we would like to calculate a probability of finding such patterns whose Chebyshev distance $d[\overrightarrow{\mathrm{X}}(i), \overrightarrow{\mathrm{X}}(j)]$ does not exceed a positive real number $r$ :

$$
C_{i}^{d_{E}}(r)=\left(N-d_{E}+1\right)^{-1} \sum_{j=1}^{N-d_{E}+1} \mathcal{H}(r-d[\overrightarrow{\mathrm{X}}(i), \overrightarrow{\mathrm{X}}(j)])
$$

where $\mathcal{H}(\cdot)$ is the Heviside function which count the number of instances $d[\overrightarrow{\mathrm{X}}(i), \overrightarrow{\mathrm{X}}(j)] \leq r$.

Next, we estimate

$$
F^{d_{E}}(r)=\left(N-d_{E}+1\right)^{-1} \sum_{i=1}^{N-d_{E}+1} \ln \left(C_{i}^{d_{E}}(r)\right),
$$

and ApEn of a corresponding time series (for fixed $d_{E}$ and $r$ ) measures the logarithmic likelihood that patterns that are close for $d_{E}$ adjacent observations remain close on the next comparison:

$$
\operatorname{ApEn}\left(d_{E}, r, N\right)=F^{d_{E}}(r)-F^{d_{E}+1}(r),
$$

i.e., equation (6) measures the logarithmic likelihood that sequences of patterns that are close for $d_{E}$ observations will remain close after further comparisons. Therefore, if the patterns in the sequence remain close to each other (high regularity), the ApEn becomes small, and hence, the time series data has a lower degree of randomness. High values of ApEn indicate randomness and unpredictability. But it should be considered that ApEn results are not always consistent, thus it depends on the value of $r$ and the length of the data series. However, it remains insensitive to noise of magnitude if the values of $r$ and $d_{E}$ are sufficiently good, and it is robust to artifacts and outliers. Although ApEn remains usable without any models, it also fits naturally into a classical probability and statistics frameworks, and, generally, despite its shortcomings, it is still the applicable indicator of system stability, which significantly increased values may prognosticate the upcoming changes in the dynamics of the data.

The empirical results for the corresponding measure of entropy along with two periods of BTC are presented in figure 8.

Long before the crisis, the value of this type of entropy begins to decrease, the complexity of the system decreases. This measure, in our opinion, is one of the earliest precursors of the crisis.

\subsubsection{Permutation entropy}

PEn, according to the previous approach, is a complexity measure that is related to the fundamental Information theory and entropy proposed by Shannon. Such a tool was proposed by Bandt and Pompe [127], which is characterized by its simplicity, computational speed that does not require some prior knowledge about the system, strongly describes nonlinear chaotic regimes. Also, it is characterized by its robustness to noise [128, 129] and invariance to nonlinear monotonous transformations [130]. The combination of entropy and symbolic dynamics turned out to be fruitful for analyzing the disorder for the time series of any nature without losing their temporal information. According to this method, we need to consider "ordinal patterns" that consider the order among time series and relative amplitude of values instead of individual values. For evaluating PEn, at first, we need to consider a time series $\{x(i) \mid i=1, \ldots, N\}$ which relevant details can be "revealed" in $d_{E}$-dimensional vector

$$
\overrightarrow{\mathrm{X}}(i)=\left[x(i), x(i+\tau), \ldots, x\left(i+\left(d_{E}-1\right) \tau\right)\right],
$$

where $i=1,2, \ldots, N-\left(d_{E}-1\right) \tau$, and $\tau$ is an embedding delay of our time delayed vector. After it, we consider $d_{E}$ ! permutation patterns $\pi=\left(k_{0}, k_{1}, \ldots, k_{d_{E}-1}\right)$ of symbols $\left(0,1, \ldots, d_{E}-1\right)$ if the following condition for each $\overrightarrow{\mathrm{X}}(i)$ 


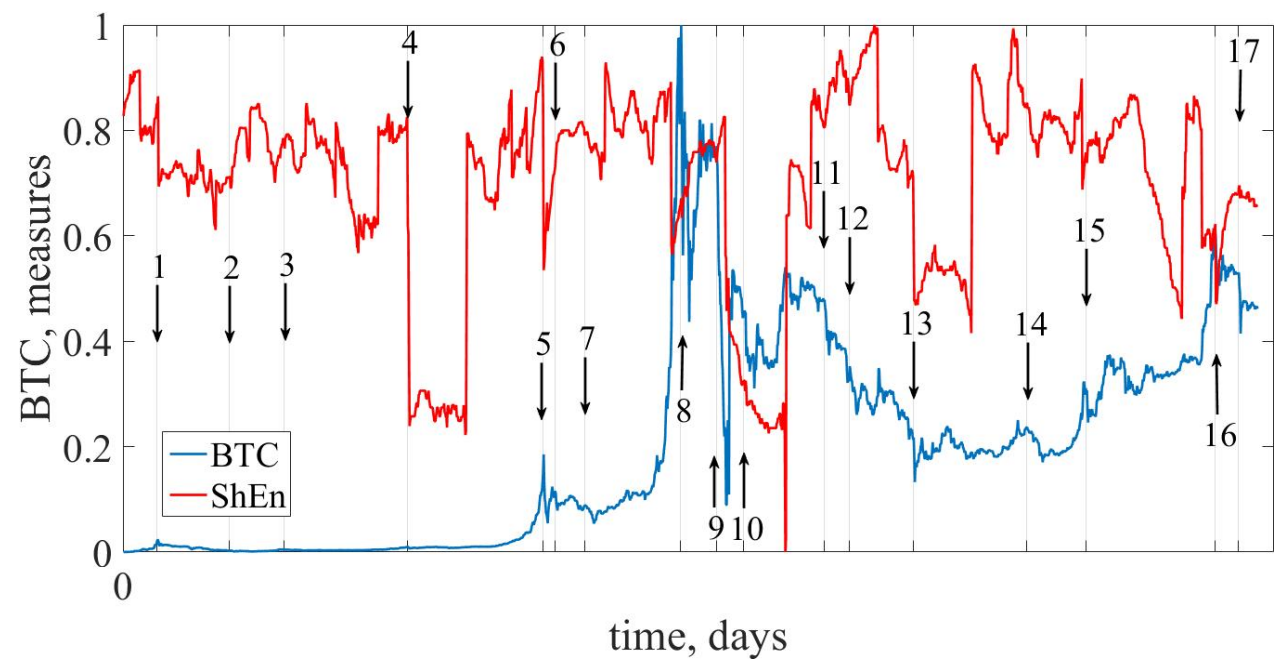

(a)

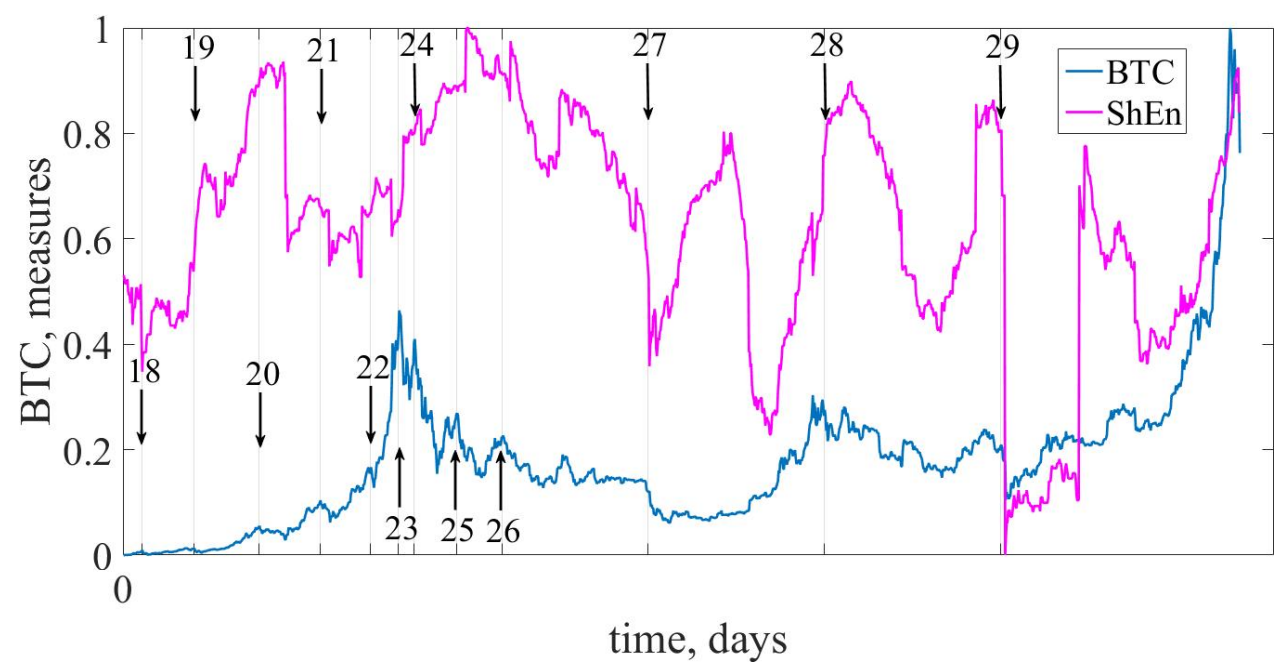

(b)

Figure 7: ShEn dynamics along with the first (a) and the second (b) periods of the entire time series of Bitcoin

is satisfied:

$$
x\left(i+k_{0}\right) \leq x\left(i+k_{1}\right) \leq \ldots \leq x\left(i+k_{d_{E}-1}\right)
$$

We will use ordinal pattern probability distribution as a basis for entropy estimation. Further, let us denote $f\left(\pi_{l}\right)$ as the frequency of occurrence of the pattern $\pi_{l}$. Then, the relative frequencies of permutations in the time series are defined as

$$
p\left(\pi_{l}\right)=\frac{f\left(\pi_{l}\right)}{N-\left(d_{E}-1\right) \tau},
$$

where the ordinal pattern probability distribution is given by $P=\left\{p\left(\pi_{l}\right) \mid l=1, \ldots, d_{E} !\right\}$. Finally, permutation entropy (denoted by $S[P]$ ) of the corresponding time series presented in the following form:

$$
S[P]=-\sum_{l=1}^{d_{E} !} p\left(\pi_{l}\right) \log p\left(\pi_{l}\right)
$$

Then, to get more convenient values, we calculate Normalized permutation entropy as:

$$
E_{S}[P]=\frac{S[P]}{S_{\max }},
$$

whose $S_{\text {max }}=\ln d_{E}$ ! represents the maximum value of $E_{S}[P]$ (a normalization constant), and normalized entropy has a range $0 \leq P E n \leq 1$. Here, the maximal entropy possible value is realized when all $d_{E}$ ! possible permutations have an equal probability of occurrence (more noise and random data). With the much lower entropy value, we get a more predictable and regular sequence of the data. Therefore, the $P E n$ gives a measure of the departure of the time series from a complete noise and stochastic time series.

There must be predefined appropriate parameters on which $P E n$ relying, namely, the embedding dimension $d_{E}$ is paramount of importance because it determines $d_{E}$ ! possible states for the appropriate probability distribution. With small values such as 1 or 2 , parameter $d_{E}$ will not 


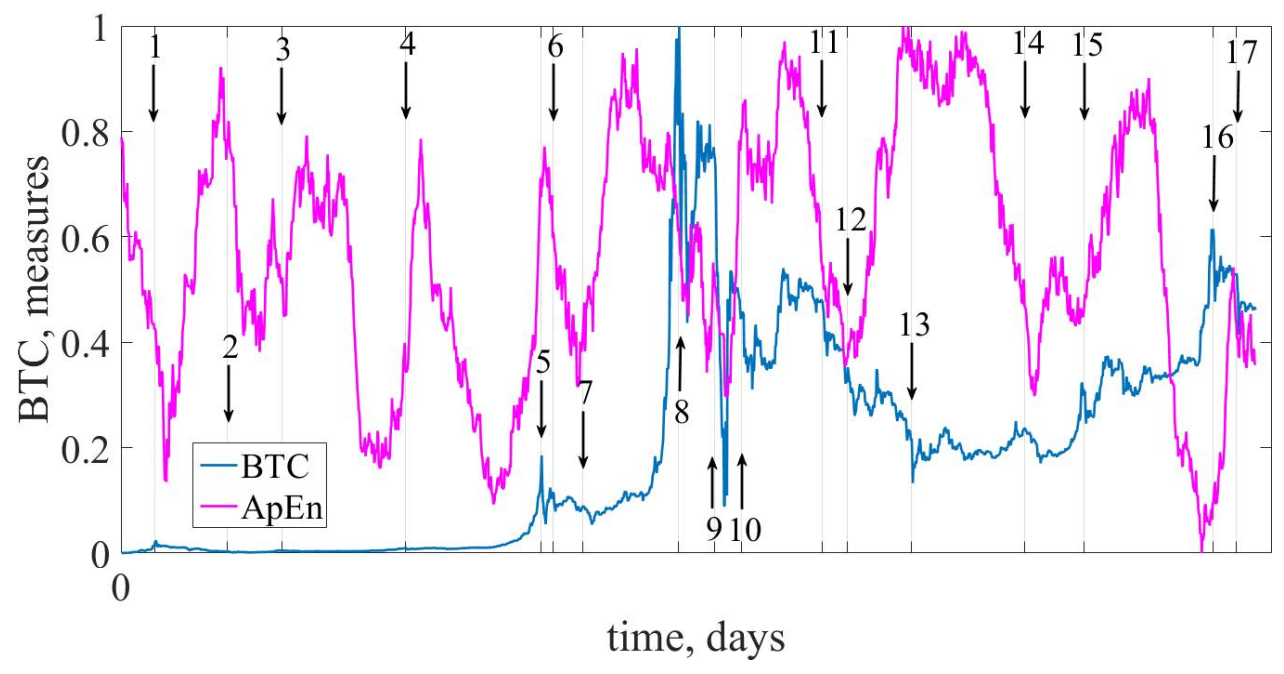

(a)

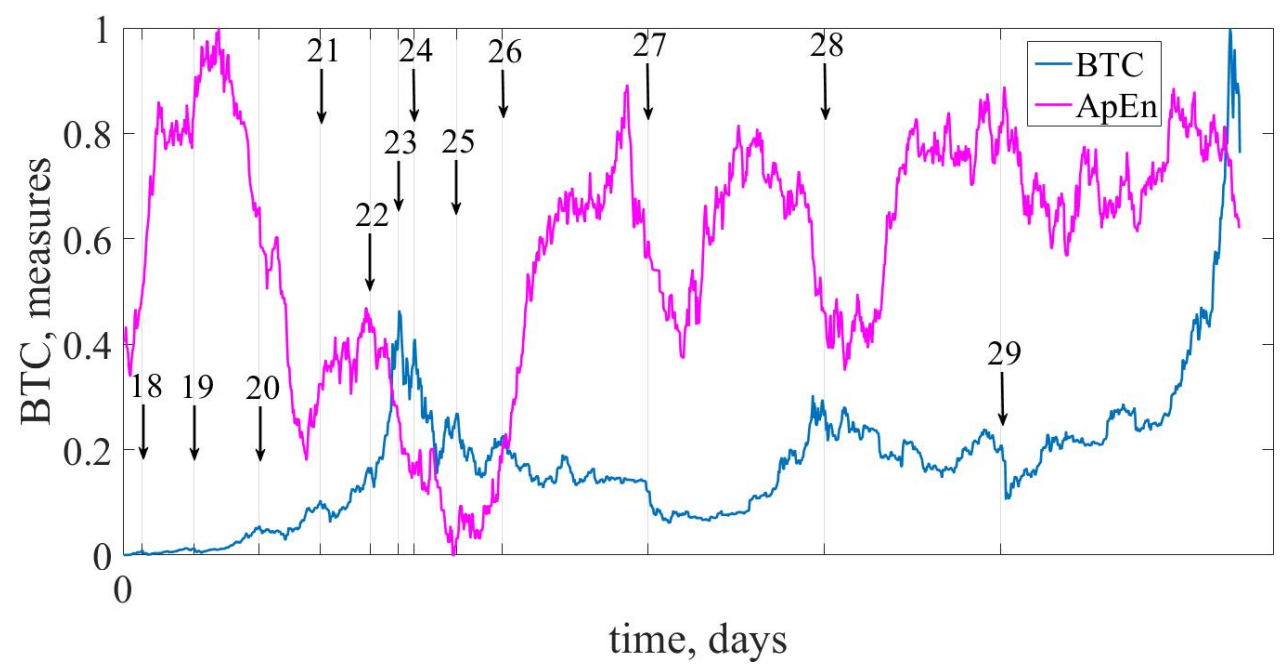

(b)

Figure 8: ApEn dynamics along with the first (a) and the second (b) periods of the entire time series of Bitcoin calculated with rolling window of 100 days and the step size of 1 day

work because there are only few distinct states. Furthermore, for obtaining reliable statistics and better detecting the dynamic structure of data, $d_{E}$ should be relevant to the length of the time series or less [140]. For our experiments, $d_{E} \in\{3,4\}$ and $\tau \in\{2,3\}$ indicate the best results. Hence, in figure 9 we can observe the empirical results for permutation entropy, where it serves as indicator-precursor of the possible crashes and critical events.

Information measures of complexity due to their initial validity and transparency, ease of implementation and interpretation of the results occupy a prominent place among the tools for the quantitative analysis of complex systems.

\section{Fractal and multifractal measures of complexity}

The economic phenomena that cannot be explained by the traditional efficient market hypothesis can be explained by the fractal theory proposed by Mandelbrot [141]. Before, fractal studies focus on the Rescaled Range (R/S) analysis were proposed by Hurst [142, 143] in the field of hydrology. However, Lo [144] discovered that the R/S method is sensitive to short-term autocorrelation, which may lead to a bias error of nonstationary time series. To solve this problem, Penget et al. [145] proposed a widely used detrended fluctuation analysis (DFA) that uses a long-range power law to avoid significant long-term autocorrelation false detection [146]. As a multifractal extension (MF) of the DFA approach, Kantelhardt et al. [147] introduced the MF-DFA method that for a long time has been successfully applied for a variety of financial markets, such as stock [148-156], commodity [154, 157-160], tanker [161], derivative [162], foreign exchange rates [163-167], and electricity markets [168]. An especially interesting application of multifractal analysis is measuring the degree 


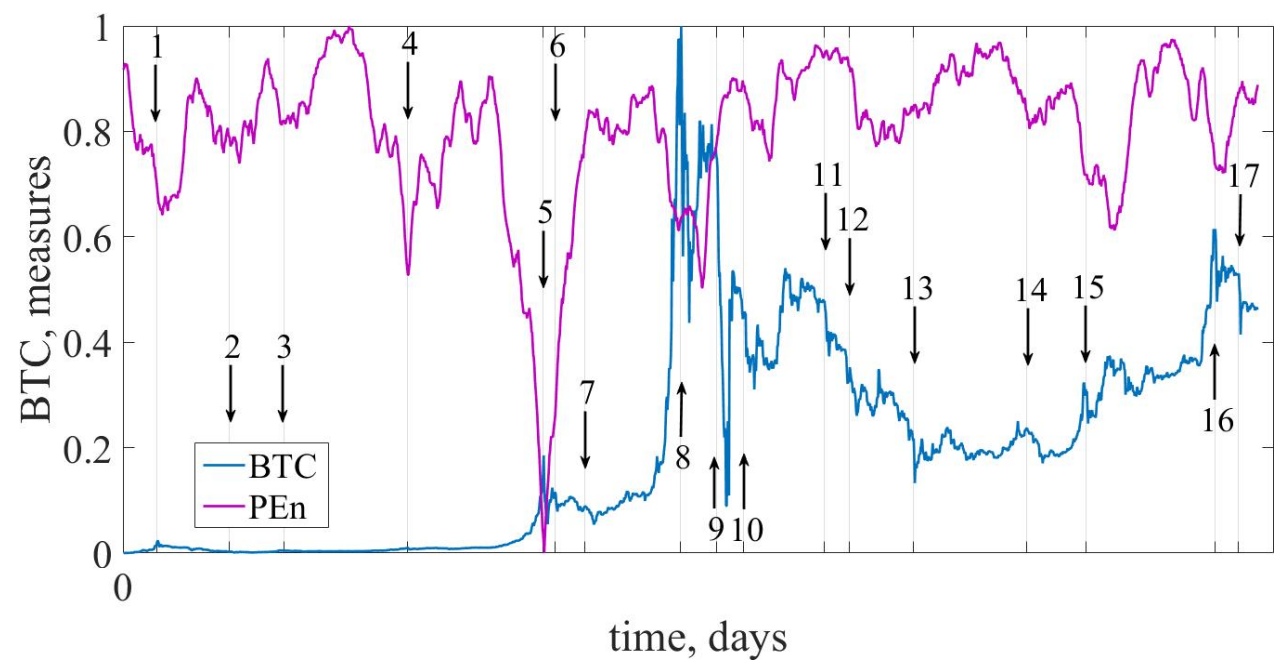

(a)

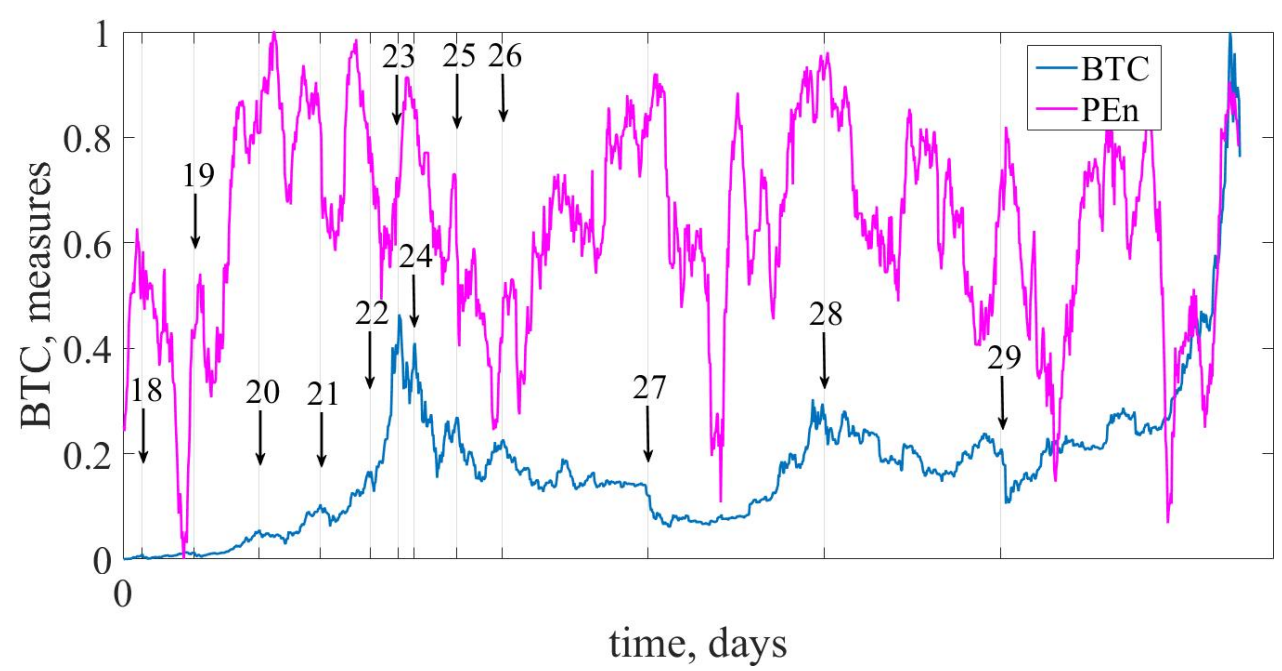

(b)

Figure 9: PEn dynamics along with the first (a) and the second (b) periods of the entire time series of Bitcoin

of multifractality of time series, which can be related to the degree of efficiency of financial markets [169-172].

Podobnik and Stanley [173] extended DFA by introducing a detrended cross-correlation analysis (DCCA) approach that can be used to study long-range crosscorrelation between two non-stationary time series. Guided by ready-made approaches, Zhou [174] proposed a multifractal detrended cross-correlation analysis (MFDCCA) [175], which is a combination of MF-DFA and DCCA. Then the number of interesting methods has been proposed, such as the method of MF-PX-DFA and MFPX-DMA [176], MF-X-DMA [177], MF-HXA [178], MF-X-PF [179], etc. These increase the efficiency of some applications of the MF-DCCA method. The MF-DCCA method has been widely applied to describe the multifractal characteristics of two cross-correlated nonstationary time series in the financial field such as the foreign exchange market [180,181], the stock market [182-184], the crude oil market [185-187], carbon market [188, 189], and the commodity market $[154,190]$. Zhang et al. also employ MF-DCCA to examine the relationship between mass media and new media [191] and to quantify crosscorrelation between investor sentiment proxies [192], i.e., fears [193] and Twitter happiness sentiment [194].

Along with common multifractal methods, Sattarhoff and Gronwald [195] applied an intermittency coefficient for the evaluation of financial market efficiency. While the random walk corresponds to the most genuine form of market efficiency, the larger the value of the intermittency coefficient is, the more inefficient a market would be. In an empirical application using data from the largest current market for tradable pollution permits, the European Union Emissions Trading Scheme, they show that this market becomes more efficient over time. Besides, the degree of market efficiency is overall similar to that for the US stock market; for one sub-period, the market efficiency is found to be higher. While the first finding is anticipated, the second finding is noteworthy, as various observers expressed 
regarding the information efficiency of this newly established artificial market.

Since Bitcoin was born, it has attracted the considerable attention of researchers from different fields of science that apply modern methods and models of analysis of the peculiarities of the dynamics of the popular digital currency, namely, the methods of multifractal analysis to gain a deeper understanding of its inherent nonlinear statistical properties.

Using 1-min returns of Bitcoin price, Takaishi investigated statistical properties and MF of Bitcoin time series [196]. His results present that 1-min returns distribution is fat-tailed, and kurtosis largely deviates from the Gaussian expectation. Although with large time scales, kurtosis is anticipated to approach the Gaussian expectation, he found that convergence to that is very slow. Skewness is found to be negative at short time scales and becomes consistent with zero at large time scales. Also, he analyzed daily volatility-asymmetry by using GARCH, GJR, and RGARCH models and found no evidence of volatility asymmetry. On exploring MF using MF-DFA, it was confirmed that the Bitcoin time series exhibits MF. The sources of MF are also investigated, and it is confirmed that both temporal correlation and the fat-tailed distribution contribute to the MF, and the degree of MF for the temporal correlation is stronger than that for the fat-tailed distribution.

Generally, regarding these papers [65, 66], Bariviera et al. investigated the long memory of the Bitcoin market using the Hurst exponent. Their research proves the advantages of the DFA methods, basically, because it is more robust and less sensitive to departures from conditions of stationarity. They find that daily returns suffered a regime switch. From 2011 until 2014 the Hurst exponent was showing persistence behavior, whereas after 2014, it equals to white noise, while daily volatility exhibits persistent behavior during the period under study. Also, daily volatility presents stronger fluctuations than in daily returns. In particular, that volatility characteristic is the main peculiarity of the Bitcoin market.

Kirichenko et al. [197] conducted a comparative correlation and fractal analysis to time series of the Bitcoin cryptocurrency rate and community activities in social networks associated with famous cryptocurrency. The results of their study show a significant correlation and similar multifractal structure between the Bitcoin rate and the community activities. Time series fractal analysis indicated the presence of self-similar and multifractal properties.

As an example, Hong et al. [198] attempt to investigate the time-varying long-term memory in the Bitcoin market through a sliding window approach and by employing a new efficiency index [199]. The daily dataset for the period from 2010 to 2017 is utilized, and some interesting findings emerge that:

- generalized Hurst exponents in the Bitcoin market are above 0.5 ;

- long-term memory exists in the Bitcoin market;

- high degree of inefficiency ratio;
- Bitcoin market does not become more efficient over time;

- rolling window approach can help to obtain more reliable results. Some conclusions for those who deal with the cryptocurrency market were made.

The Al-Yahyaee et al. [200] paper provides the results on the efficiency of the Bitcoin market compared to gold, stock, and foreign exchange markets. By applying the MFDFA approach, researchers found that the long-memory feature and MF of the Bitcoin market were more robust and, therefore, more inefficient than the gold, stock, and currency markets.

Gajardo et al. [201] applied MF-ADCCA to analyze the presence and asymmetry of the cross-correlations between the major currency rates, Bitcoin, the DJIA, gold price, and the oil crude market. They found that multifractality existed in every cross-correlation studied, and there was an asymmetry in the cross-correlation exponents under the different trends of the WTI, Gold, and the DJIA. Bitcoin showed greater multifractal spectra than the other currencies on its cross-correlation with the WTI, the Gold, and the DJIA. Bitcoin presented a different relationship between commodities and stock market indices, which had to be taken into consideration when investing. The reason is that over the years the currency was traded and over time, it has earned the trust of the community.

The nonlinear patterns of the volatility of the seven Bitcoin markets were investigated by Lahmiri et al. [202]. Using four diverse distribution inferences: Normal, Student-t, Generalized Error, and t-Skewed distribution, they explored the fractional long-range dependence in conjunction with the potential inherent stochasticity of volatility time series. Their results testify to the existence of long-range memory in Bitcoin market volatility, irrespectively of distributional inference. The entropy measurement, which indicates a high degree of randomness in the estimated series, shows the same. As Bitcoin markets are highly disordered and risky, they cannot be considered suitable for hedging purposes. Their exploration provides strong evidence against the efficient market hypothesis.

Wei Zhang et al. [203] investigated the crosscorrelations of the return-volume relationship of the Bitcoin market. In particular, they selected eight exchange rates whose trading volume accounts for more than 98\% market shared to synthesize Bitcoin indexes. The empirical results based on MF-DCCA revealed that: (1) return-volume relationship exhibited the nonlinear dependencies and power-law cross-correlations; (2) all cross-correlations were multifractal, and there were antipersistent behaviors of cross-correlation for $q=2$; (3) the price of small fluctuations was more persistent than that of the volume, while the volume of larger fluctuations was more anti-persistent; (4) the sliding window approach showed that the cross-correlations of return-volume were anti-persistent in the entire sample period.

Similarly to our article [204] where we applied the MF-DFA method to Ukrainian and Russian stock markets, we use it here to explore the multifractal property of Bitcoin and construct reliable indicator for it. 


\subsection{Multifractal detrended fluctuation analysis (MF-DFA)}

As an extension to the original DFA [145, 205, 206], the multifractal approach $[147,207]$ estimates the Hurst exponent of a time series at different scales. Based on a given time series $\{x(i) \mid i=1, \ldots, N\}$, the MF-DFA is described as follows:

(i) The profile $Y(i)$ (accumulation) is defined as:

$$
Y(i)=\sum_{j=1}^{i}(g(j)-\langle g\rangle),
$$

where $\langle g\rangle$ stands for the average of returns.

(ii) The profile $Y(i)$ is then divided into $N_{s} \equiv \operatorname{int}(N / s)$ non-overlapping time segments of equal length $s$ and the local trend $Y_{v}^{f i t}$ for each segment is calculated by the least-square fit. Since the length of the time series is not always a multiple of $s$, a short period at the end of the profile, which is less than the window size, may be removed. For taking into account the rejected part and, therefore, to use all the elements of the sequence, the above procedure is repeated starting from the end of the profile. Therefore, the total $2 N_{s}$ segments are obtained together, and the variance is computed as

$$
\begin{array}{r}
F^{2}(v, s)=\frac{1}{s} \sum_{i=1}^{s}\left[Y((v-1) s+i)-Y_{v}^{f i t}(i)\right]^{2}, \\
\text { for } v=1, \ldots, N_{s}
\end{array}
$$

and

$$
\begin{array}{r}
F^{2}(v, s)=\frac{1}{s} \sum_{i=1}^{s}\left[Y\left(N-\left(v-N_{s}\right) s+i\right)-Y_{v}^{f i t}(i)\right]^{2}, \\
\text { for } v=N_{s}+1, \ldots, 2 N_{s}
\end{array}
$$

Various types of MF-DFA such as linear, quadratic, or higher order polynomials can be used for eliminating local trend in segment $v$; we use a cubic order polynomial.

(iii) Considering the variability of time series and the possible multiple scaling properties, we obtain the $q^{\text {th }}$ order fluctuation function by averaging over all segments:

$$
F_{q}(s)=\left[\frac{1}{2 N_{s}} \sum_{v=1}^{2 N_{s}}\left[F^{2}(v, s)\right]^{q / 2}\right]^{1 / q} .
$$

The index $q$ can take any non-zero value. For $q=0$, $F_{q}(s)$ is divergent and can be replaced by an exponential of a logarithmic sum

$$
F_{0}(s)=\exp \left[\frac{1}{4 N_{s}} \sum_{v=1}^{2 N_{s}} \ln F^{2}(v, s)\right] .
$$

(iv) At least, we determine the scaling behavior of the fluctuation function by analyzing $\log F_{q}(s)$ vs $\log s$ graphs for each value of $q$. Here, $F(s)$ is expected to reveal power-law scaling

$$
F_{q}(s) \sim s^{h(q)}
$$

for large $n$. The scaling exponent $h(q)$ can be considered as generalized Hurst exponent. With $q=2$ MFDFA transforms into standard DFA, and $h(2)=H$, where $H$ is the well-known Hurst exponent.

- $h(2) \in[0.0,0.5) \rightarrow$ anti-persistency. The process under study tends to decrease (increase) after a previous increasing (decreasing);

- $h(2)=0.5 \rightarrow$ uncorrelated process. The fluctuations that depend on $q$ tend to a random walk behavior [131];

- $h(2) \in(0.5,1.0] \rightarrow$ persistency. If a process tends to increase (decrease) for a some period $T$, then it expected to continue to increase (decrease) for a similar period of time;

- $h(2)>1.0 \rightarrow$ nonstationary process, stronger long-range correlations are present.

Those intervals with time intervals $v$ will dominate which variance $F^{2}(v, s)$ is large and $q$ values are positive. Therefore, for positive values of $q, h(q)$ describes the scaling behavior of time intervals with large fluctuations. Large fluctuations are usually characterized by smaller scaling coefficients of $h(q)$ for multifractal series. On the contrary, for negative values of $q$, time intervals with a small variance $F^{2}(v, s)$ will dominate. Thus, $h(q)$ will describe the scaling behavior of time intervals with small fluctuations.

(v) Another way of characterizing multifractality of a time series is in terms of the multifractal scaling exponent $\tau(q)$ which is related to the generalized Hurst exponent $h(q)$ from the standard multifractal formalism and given by [146]:

$$
\tau(q)=q h(q)-1 .
$$

Equation (12) reflects temporal structure of the time series as a function of moments $q$, i.e., it represents the scaling dependence of small fluctuations for negative values of and large fluctuations for positives values. If (12) represents linear dependence of $q$, the time series is said to be monofractal. Otherwise, if (12) has a nonlinear dependence on $q$, then the series is multifractal.

(vi) The different scalings are better described by the singularity spectrum $f(\alpha)$ which can be defined as:

$$
\begin{gathered}
\alpha=\frac{d \tau(q)}{d q}=h(q)+q \frac{d h(q)}{d q}, \\
f(\alpha)=q \alpha(q)-\tau(q)=q^{2} \frac{d h(q)}{d q}+1,
\end{gathered}
$$


with $\alpha$ - the Hölder exponent or singularity strength. Following the methods described above, we present results that reflect multifractal behavior of the Bitcoin time series.

Figure 10a presents $F_{q}(s)$ in the log-log plot. The slope changes dependently on $q$, which indicates the multifractal property of a time series. As it was pointed out, multifractality emerges not only because of temporal correlation, but also because the Bitcoin returns distribution turns out to be broad (fat-tailed) [147], and this distribution could contribute to the multifractality of the time series. The same dependence can be observed in the remaining plots. The scaling exponent $\tau(q)$ remains nonlinear, as well as generalized Hurst exponents that can serve as evidence that Bitcoin exhibit multifractal property.

In the case of multifractals, the shape of the singularity spectrum typically resembles an inverted parabola (see figure 10d); furthermore, the degree of complexity is straightforwardly quantified by the width of $f(\alpha)$, simply defined as $\Delta \alpha=\alpha_{\max }-\alpha_{\min }$, where $\alpha_{\max }$ and $\alpha_{\min }$ correspond to the opposite ends of the $\alpha$ values as projected out by different $q$-moments (equation (10)).

In the figure below we present the width of the spectrum of multifractality that changes over time accordingly to the sliding window approach. The whole figure consists of both a three-dimensional plot (singularity spectrum) and two-dimensional representation of its surface.

If the series exhibited a simple monofractal scaling behavior, the value of singularity spectrum $f(\alpha)$ would be a constant. As can be observed, here our series exhibits a simple multifractal scaling behavior, as the value of singularity spectrum $f(\alpha)$ changes dependently on $\alpha$, i.e., it exhibits different scalings at different scales. Moreover, with the sliding window of the corresponding length, we understand that at different time periods Bitcoin becomes more or less complex. The value of $\Delta \alpha$ gives a shred of additional evidence on it.

As we can see from the presented results, the width of the singularity spectrum after the crisis starts to increase, which tells us that more violent price fluctuations are usually expected. With the decreasing width of the singularity spectrum, the series is expected to hold the trend. As the rule, it reaches its minimum before the collapse of the BTC price.

\section{Chaos-dynamical measures}

Apparently random fluctuations in financial systems often tend to exhibit varying levels of complexity and chaos. Regarding limited data, it becomes hard to define the boundaries of predictability of them. The analysis of such systems, the processes driving their dynamics chaos theory has been considered in various fields such as economics, finance, physics, and others [208-213]. Regarding the analysis of Bitcoin dynamics, the knowledge about its completely random and, at the same time, deterministic processes can potentially explain fluctuations in time series of different nature. Considering the financial sector, evidence on deterministic chaos, the knowledge of such moments when two initially close trajectories start to diverge, and of the periods for which they will stay close to each other would have important implications for regulators and traders, who will develop effective short-term trading strategies. During the years, chaos theory has been providing approaches to study some interesting properties of time series. The most widespread are: correlation dimension, the BDS test, Kolmogorov entropy, Lyapunov exponent, close returns test, etc. [214-217].

Endowing Bitcoin time series with the sliding window approach and efficient methods of Lyapunov exponents, and Levy alpha-stable distribution, we are going to reflect its transition between chaotic and non-chaotic behavior. Also, as it has been observed, such unstable events as market crashes correspond to fat tails. Thus, the analysis of such extreme events can be understood throughout Levy alpha-stable distribution.

\subsection{Lyapunov exponents}

The evolution of the system exhibits sensitive dependence on initial conditions. It means that initially close trajectories that evolve may rapidly diverge from each other and have totally different outcomes. Accordingly, with small uncertainties that amplify enormously quickly, long-term predictions turn out to be impossible. On the other hand, in a system with attraction points or stable points, the distance between them decreases asymptotically in time or with the number of points, which tend to converge [218].

To present the idea more precisely, let's consider two consecutive points presented as $x(t)$ and its initially close neighbor as $x(t)+\delta(t)$, where $\delta(t)$ represents a tiny deviation in time $t$, as presented in figure 13

As two initially close points disturbed by some event, they start to diverge, and the distance between them grows following exponential law:

$$
\|\delta(t)\| \approx\|\delta(0)\| \exp (\lambda t)
$$

with $\lambda$ that denotes the Lyapunov exponent (LE); $\delta(t)$ is the distance between the reference point and its nearest neighbor after $t$ iterations; $\delta(0)$ is the initial distance between the reference point and its nearest neighbor perturbed with a small error at $t=0$.

LE is a measure of the exponential rate of nearby trajectories in the phase-space of a dynamical system. In other words, it quantifies how fast converge or diverge trajectories that start close to each other, quantifying the strength of chaos in the system.

In such cases when our system $n$-dimensional, we have as many LEs as the dimensions in it. To define them, we consider the evolution of an infinitesimal sphere of perturbed initial conditions. During its evolution, the sphere will become distorted into an infinitesimal ellipsoid. Defining the length of the $i^{t h}$ principal axis as $\delta_{i}(t)$, there are $n$-Lyapunov exponents given by:

$$
\left\|\delta_{i}(t)\right\| \approx\left\|\delta_{i}(0)\right\| \exp \left(\lambda_{i} t\right), \quad \text { for } i=1, \ldots, n .
$$

To identify whether the motion is periodic or chaotic, especially, for large $t$ it is recommended to contribute to 


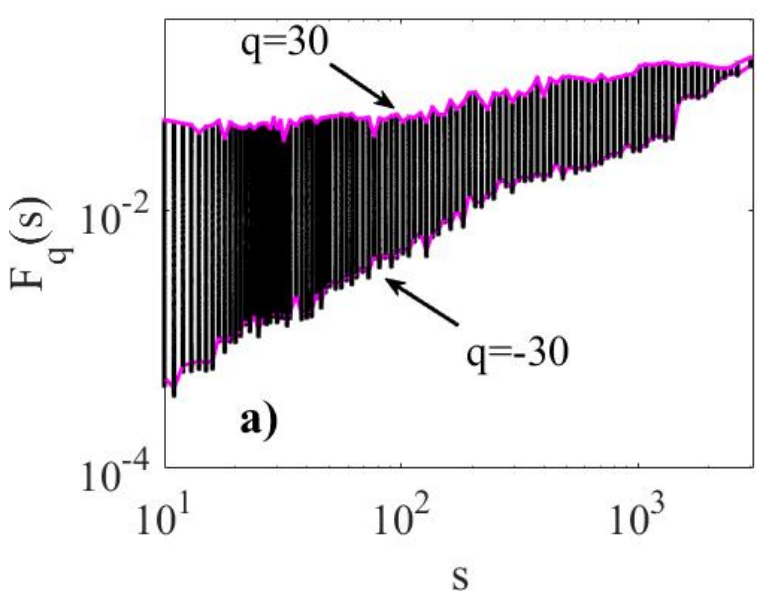

(a)

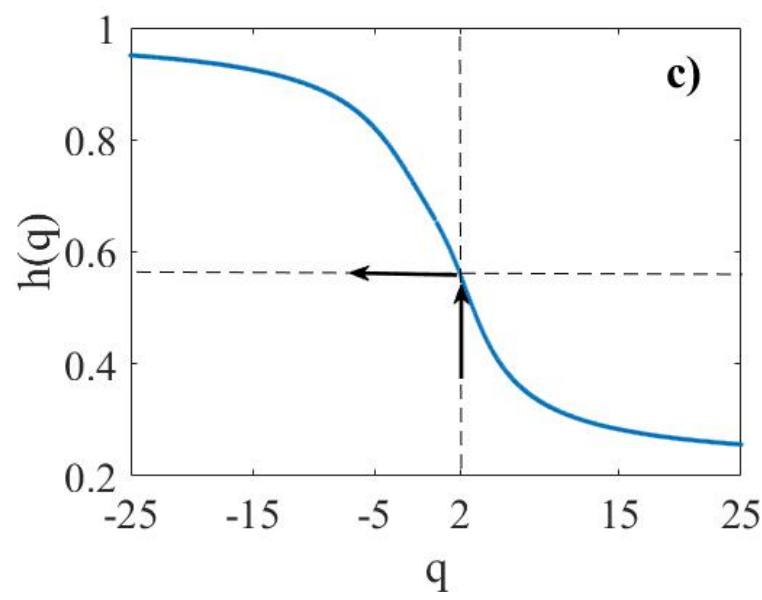

(c)

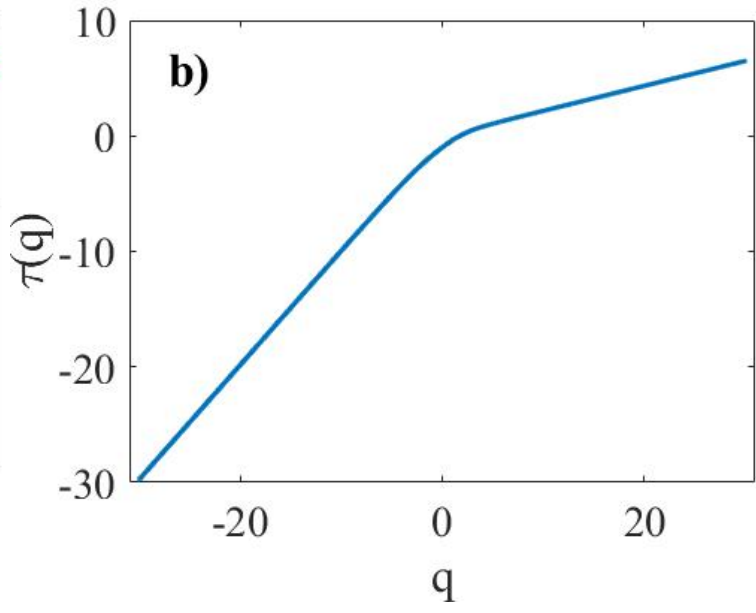

(b)

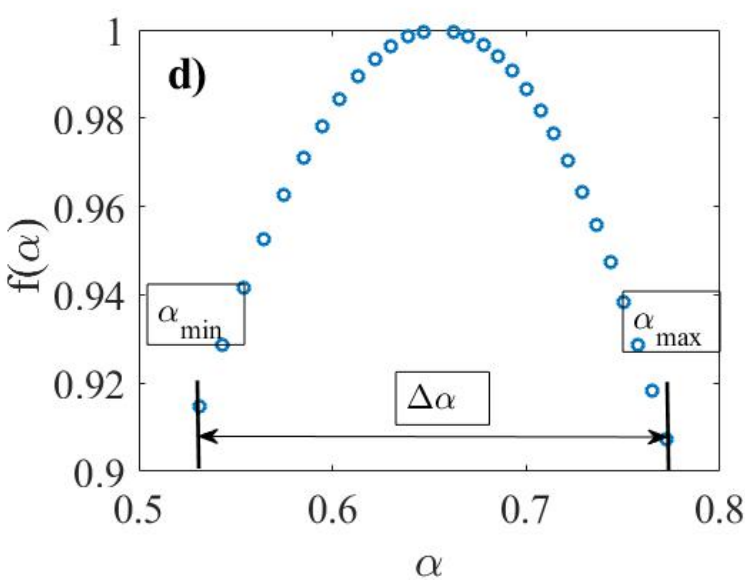

(d)

Figure 10: The fluctuation function $F_{q}(s)$ (a), multifractal scaling exponent $\tau(q)(\mathrm{b}), h(q)$ versus $q$ of the BTC return series (c), and singularity spectrum $f(\alpha)(\mathrm{d})$ obtained from MF-DFA for BTC time series.

the largest Lyapunov exponent (LLE) among the others of the $n$-dimensional dynamical system [219], as the diameter of ellipsoid starts to be controlled by it. Exactly the LLE is used to quantify the predictability of the systems, since exponential divergence means that in the system where the initial difference was infinitesimally small, start to rapidly lose its predictability behaving differently. However, it should be noted that other exponents also contain important information about the stability of the system, including the directions of convergence and divergence of the trajectories [220].

The existence of at least one positive LE is generally seen as a strong indicator of chaos. Positive LE means that initially similar, phase space trajectories that are sensitive to initial conditions and diverge exponentially fast, characterize chaotic behavior of the system. Negative LE responds to the cases when trajectories remain close to each other, but it is not necessarily implied stability, and we have to examine our system in more detail. Zero or very close to zero exponents indicate that perturbations made along the trajectory neither diverge nor converge.
With the great interest in LE, more and more methods and proposals for their calculating have appeared. Unfortunately, there has not been obtained accepted and universal method for estimating the whole spectrum of Lyapunov exponents from a time series data. One of the most common and popular algorithms have been applied by Wolf et al. [221], Sano and Sawada [222], and later improved by Eckmann et al. [223], Rosenstein et al. [224], Parlitz [225], and Balcerzak et al. [226]. Here, we followed the methods proposed by Eckmann and Gao et al. [81, 227$230]$ to compute the spectrum of Lyapunov exponents. With Rosenstein's algorithm, we compute only the LLE from an experimental time series. As again suggested by Eckmann et al. [231] one of the measures from recurrence quantification analysis can be considered for estimation of the LLE since it detects in a similar way highly nonmonotonic behavior.

With the high growth in computer science, computer simulations of complex and chaotic systems become increasingly appreciated. For at least two decades, with development in numerical computations and quantitative 


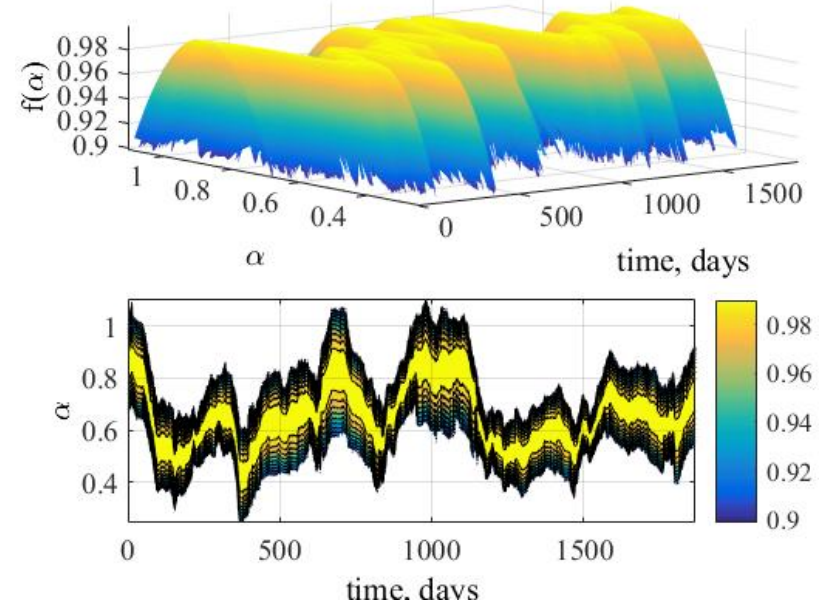

Figure 11: Changes in the spectrum of multifractality in time

analysis, no doubt left that chaos theory suggests the same unstable fluctuation that may be as common as the extreme events and critical transitions in financial markets. For instance, Scheinkman and LeBaron [232] explored several indications of nonlinear dynamic structure in stock market returns. In their opinion, the weaknesses of such studies are based on time series that are not long enough to reveal the strange (fractal) attractors. On the other hand, the reason may be chaos that comprises a class of signals intermediate between regular periodic or quasiperiodic motions and unpredictable, truly stochastic behavior [233]. Kulkarni [234] denotes that, probably, random financial fluctuations often exhibit varying levels of fluctuations, chaos. Kulkarni's paper represents the efficiency of LE for the complexity analysis of shortly limited data. The analysis constitutes weakly chaotic behavior which alternates with non-chaotic over the entire period of analysis.

Lyapunov exponents are a natural first choice in exploring and indicating such chaotic behaviors that occur in it. They do not only classify the system but also tell us the limits of predictability of the chaotic system [233]. During the last few decades, there was plenty of scientific research that was related to chaos systems, chaos behavior and, namely, to the LE. The earliest papers, in which authors $[235,236]$ try to use LE to detect chaos dynamics in financial time series, it is determined that linear, deterministic processes are characterized with negative LE from nonlinear, chaotic processes with the largest exponent (where it is positive). Besides, there is an article [237] in which Gençay presents a methodology to compute the empirical distributions of LE's using a blockwise bootstrap technique. This method provides a formal test of the hypothesis that the LLE equals some hypothesizes value, and can be used to test the system for the presence of chaotic dynamics. Such methodology is particularly useful in those cases where the largest exponent is positive but very close to zero.

Sarkar and Chadha [238] in their paper investigated the local fractal and chaotic properties of financial time series by calculating two exponents, the Local Hurst Exponent and LE. As was seen in their research, all calculations were made with the algorithm of the sliding window where they had considered two major financial indices of the US: the DJIA and the S\&P 500. Regarding the considered measures, they attempted to predict the major crashes that took place in these markets.

Srinivasan et al. [239] have provided an explanation and motivation for reconstructed phase spaces using the methods of time delay and SVD embedding. They explained the meaning of LE and an algorithm for its estimation for the corresponding chaotic, deterministic, and periodic time series. From their presented results it is seen that estimated positive and zero exponents converge to the expected, documented values. Mastroeni and Vellucci [240] obtained empirical results with the help of the LLE and a determinism test that shows that commodity and futures prices are representatives of a nonlinear deterministic, rather than stochastic systems. Similarly to [238], Plakandaras et al. [241] measured the Hurst exponent and LE in the sliding window to focus on persistence and chaotic behavior - two prime characteristics of uncertainty indices. For such purpose, they analyzed 72 popular indices constructed by forecasting models, text mining from news articles, and data mining from monetary variables. More specifically, researchers found that almost all uncertainty indices are persistent, while the chaotic dynamics are detected only sporadically and for certain indices during recessions of economic turbulence. Authors of empirical analysis [242] in one of their chapters explored whether the global markets are intrinsically unstable where unpredictability, disorder, and discontinuities are inherent and not aberrations. They investigated a huge amount of literature and examine the possible nonlinear, particularly chaotic nature of the global stock markets. Their study explores the possible presence of chaos in two phases: over the period for 1998-2005 and from 2006 to 2011. Over 30 indices had been investigated. Empirical results showed that for the first phase, 29 indices are deterministic. But 10 of them are found to be non-chaotic. Estimated determinism factors for all the indices are quite high, but Lyapunov exponent is presented to be non-positive for at least 6 of them, where others are chaotic.

As it is seen, chaos theory and its tools remain a huge challenge for researchers of different fields of science and, namely, in the financial industry, and, as it was suggested in [241], the examination of persistent and chaos should be a prerequisite step before using financial indices in economic policy model. The world of Lyapunov exponents remains a growing interest in their definition, numerical methods, and application to various complex systems. In summary, LLE allows us to establish [219]:

- transition region between stable and unstable;

- stability region;

- unstable region;

- chaotic region, including a possible transition between unstable and chaotic. 


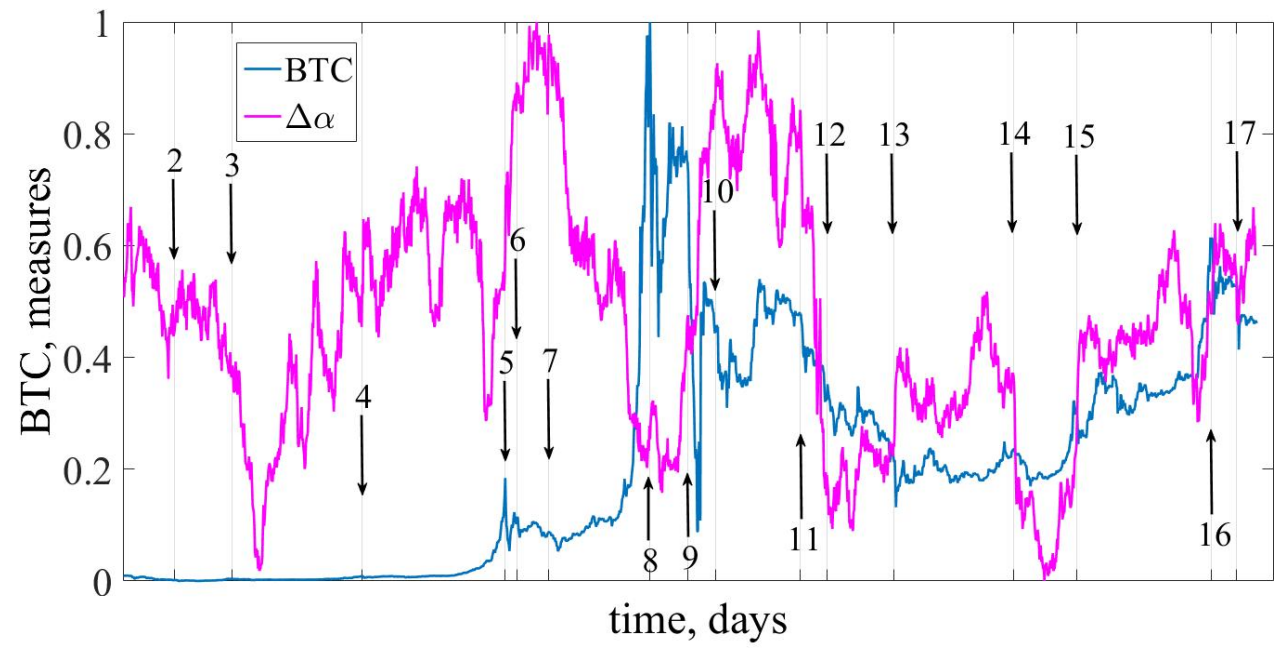

(a)

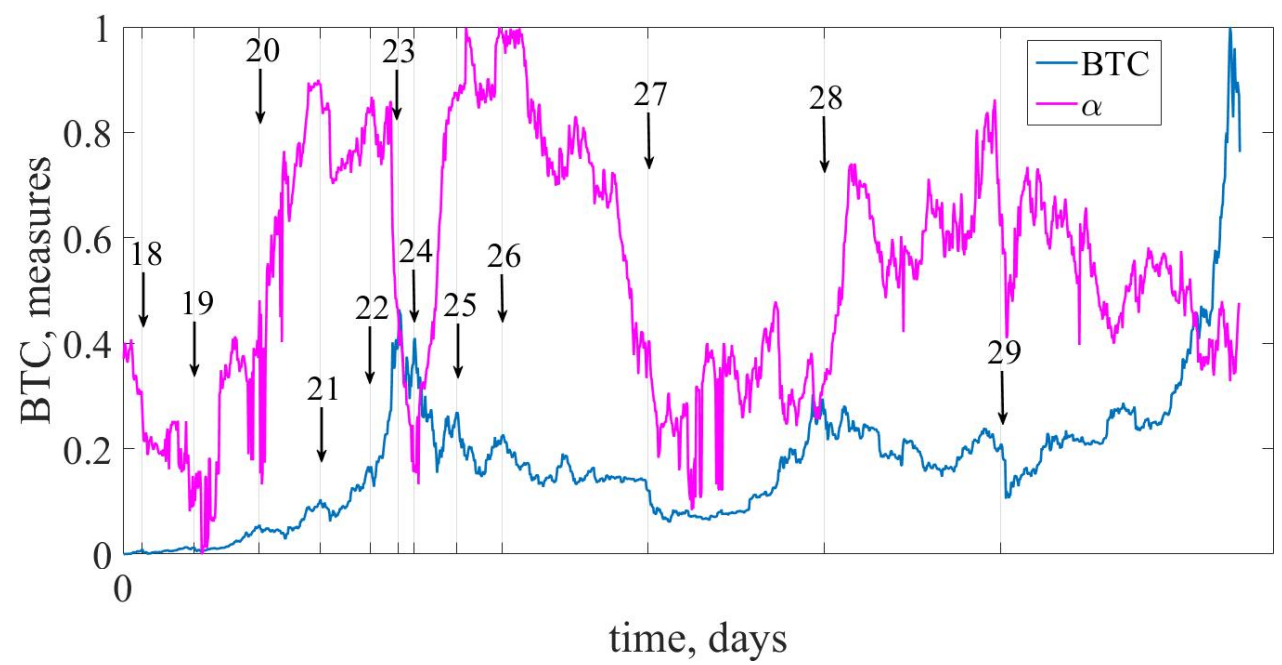

(b)

Figure 12: The comparison of the corresponding first (a) and second (b) Bitcoin time intervals with the width of the multifractality spectrum measure

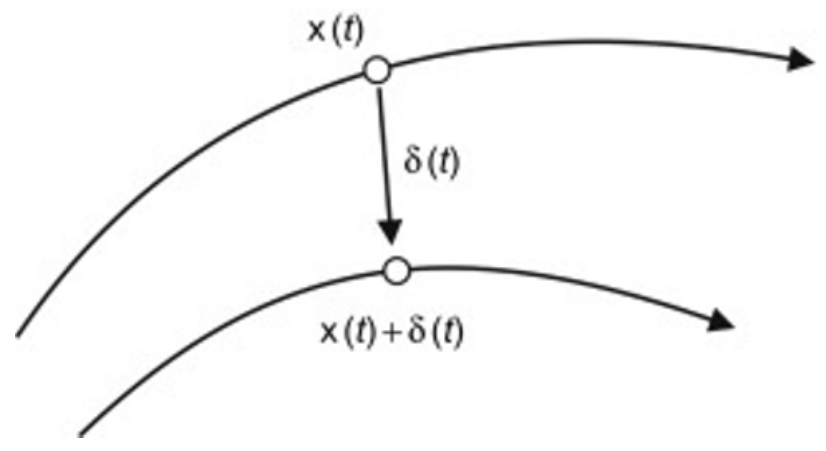

Figure 13: Divergence of two initially close trajectories in a dynamical system [219]

\subsubsection{Eckmann et al. method}

Firstly, according to the approach [223], we need to reconstruct attractor dynamics from a single time series $\{x(i) \mid i=1, \ldots, N\}$ with the embedding dimension $d_{E}$, and after this, we construct $d_{E}$-dimensional orbit representing the time evolution

$$
\begin{array}{r}
\overrightarrow{\mathrm{X}}(i)=\left[x(i), x(i+1), \ldots, x\left(i+\left(d_{E}-1\right)\right)\right], \\
\text { for } i=1, \ldots, N-d_{E}+1 .
\end{array}
$$

Then, we have to determine the most neighboring trajectories with $\vec{X}(i)$ :

$$
\|\overrightarrow{\mathrm{X}}(i)-\overrightarrow{\mathrm{X}}(j)\|=\max _{0 \leq \alpha \leq d_{E}-1}\{|x(i+\alpha)-x(j+\alpha)|\} .
$$

We sort the $x(i)$ so that $x(\Pi(1)) \leq x(\Pi(2)) \leq \cdots \leq x(\Pi(N))$ and store the permutation $\Pi$, and its inverse $\Pi^{-1}$. Then, we try to find the neighbors of $x(i)$ in dimension 1 by looking at $k=\Pi^{-1}(i)$ and scan the $x(\Pi(s))$ for $s=k+1, k+2, \ldots$ and $k-1, k-2, \ldots$ until $x(\Pi(s))-x(i)>r$. For chosen embedding dimension $d_{E}>1$, we select the value of $s$ for which further condition is true

$$
|x(\Pi(s)+\alpha)-x(j+\alpha)| \leq r, \quad \text { for } \alpha=0,1, \ldots, d_{E}-1 .
$$


After we embedded our system in $d_{E}$ dimensions, we need to determine the $d_{E} \times d_{E}$ matrix $M_{i}$ that will describe time evolution of the vectors that surround trajectory $\vec{X}(i)$, and how they map onto $\overrightarrow{\mathrm{X}}(i+1)$ state. The matrix $M_{i}$ is obtained by looking for neighbors

$$
M_{i}(\overrightarrow{\mathrm{X}}(i)-\overrightarrow{\mathrm{X}}(j)) \approx \overrightarrow{\mathrm{X}}(i+1)-\overrightarrow{\mathrm{X}}(j+1) .
$$

Nevertheless, the vectors $\vec{X}(i)-\vec{X}(j)$ may not span $\mathfrak{R}^{d_{E}}$. In this case, such indeterminacy may lead to spurious exponents which confuse the analysis. To overcome such obstacles, the projection of the trajectories is determined on a subspace of dimension $d_{M} \leq d_{E}$. Thus, the manifold on which the dynamics takes place corresponds to the local dimension $d_{M}$, where $d_{E}$ should be somewhere larger than $d_{M}$ to avoid the presence of false neighbors $[130,233]$. Hence, the trajectory $\vec{X}(i)$ is associated with a $d_{M}$-dimensional vector

$$
\begin{aligned}
\overrightarrow{\mathrm{X}}(i) & =\left[x(i), x(i+\tau), \ldots, x\left(i+\left(d_{M}-1\right) \tau\right)\right]= \\
& =\left[x(i), x(i+\tau), \ldots, x\left(i+d_{E}-1\right)\right],
\end{aligned}
$$

where $\tau=\left(d_{E}-1\right) /\left(d_{M}-1\right)$. When $\tau>1$, the condition (16) is replaced by

$$
M_{i}(\overrightarrow{\mathrm{X}}(i)-\overrightarrow{\mathrm{X}}(j)) \approx \overrightarrow{\mathrm{X}}(i+\tau)-\overrightarrow{\mathrm{X}}(j+\tau) .
$$

The $M_{i}$ is then defined by the linear least-square method [243]. The last step of the algorithm is the classical QR matrix decomposition to find orthogonal matrices $Q_{i}$ and upper-triangular matrices $R_{i}$ with non-negative diagonal elements such that

$$
M_{1+i \tau} Q_{i}=Q_{i+1} R_{i+1}, \quad \text { for } i=0,1,2, \ldots .
$$

As it was proposed by Eckmann et al. [137, 223, 244], in order to calculate $d_{M}$ Lyapunov exponents, the equation for the $k^{\text {th }}$ Lyapunov exponent with $K$ number of points on the attractor, for which the Jacobian has been estimated, the diagonal eigenvalues of the matrix $R_{i}$ and the sampling step $\Delta t$ is given by:

$$
\lambda_{k}=\frac{1}{\Delta t} \frac{1}{\tau} \frac{1}{K} \sum_{i=0}^{K-1} \ln \left(R_{i}\right)_{k k} .
$$

Thus, with linearizations by using the diagonal elements from the QR decomposition, we can calculate Lyapunov exponents.

The calculation results for the LLE on the example of BTC are presented in figure 14.

Let us pay attention to the behavior of $\lambda_{\max }$ at moments of the known failures noted in the list of crashes and critical events. Definitely, we can see that in the pre-crisis period, the value of LLE decreases markedly, then increases in the post-crisis period.

\subsubsection{Rosenstein's et al. method}

Rosenstein's algorithm [224] uses the delay embedding method that reconstructs the most important features of a multi-dimensional attractor into a single one-dimensional time series of some finite size $N$. For the time series $\{x(i) \mid i=1, \ldots, N\}$, each delay embedded vector $\overrightarrow{\mathrm{X}}(i)$ will be presented similarly to the vector (17) with embedding dimension $d_{E}$ and time delay $\tau$. Then, in the reconstructed trajectory, we initialize searching for in the state space for the nearest neighbor $\vec{X}(j)$ of the trajectory $\vec{X}(i)$ :

$$
\delta_{i}(0)=\min _{\overrightarrow{\mathrm{X}}(i)}\|\overrightarrow{\mathrm{X}}(i)-\overrightarrow{\mathrm{X}}(j)\|, \quad \text { for }|i-j|>\text { mean period, }
$$

where \|\| is the Euclidian norm, $\overrightarrow{\mathrm{X}}(j)$ is the nearest neighbor, and $\vec{X}(i)$ is the reference point.

From equation (13) we have already known that the distance between states $\overrightarrow{\mathrm{X}}(i)$ and $\overrightarrow{\mathrm{X}}(j)$ will grow in time accordingly to a power law, where $\lambda$ is a good approximation of the LLE. For further estimations, we look at the logarithm of the distance trajectory $\ln \delta_{i}(k) \approx \lambda(k \cdot \Delta t)+\ln c_{i}$, where $\delta_{i}(k)$ is the distance between $i^{\text {th }}$ pair of the nearest neighbors defined in equation (18) after $k$ time steps, $c_{i}$ is the initial separation of them, and $\Delta t$ is the time interval between measurements (sampling period of the time series).

Further result of this algorithm is not a numerical value, but a function of time

$$
y(k, \Delta t)=\frac{1}{\Delta t} \frac{1}{M} \sum_{i=1}^{M} \ln \delta_{i}(k)
$$

with the size of the reconstructed time series $M=N-$ $\left(d_{E}-1\right) \tau$, and a set of approximately parallel lines $\delta_{i}(k)$ whose slope roughly proportional to the LLE. Then, it is proposed to be calculated as the angle of inclination of its most linear section. Finding such a section turns out to be a non-trivial task, and sometimes it is impossible to specify such a section at all. Despite this problem, Rosenstein's method is easy for implementing and computing.

The LLE behavior for a window procedure with the length of 250 days and the step size of 1 day is shown in figure 15 .

It can be seen that, as before, the LLE is also sensitive to the crisis conditions of BTC.

\subsubsection{Scale-dependent Lyapunov exponent (SDLE)}

We briefly describe the idea and the formal foundations of the method SDLE, introduce new measures of complexity, and illustrate their effectiveness with the example of the BTC index. Let us have a single observation conducted at a discrete time interval $\Delta t$ in the form of a time series $\left\{x_{i} \mid i=1, \ldots, N\right\}$ where $t=i \cdot \Delta t$. After reconstructing the phase space, let us consider the ensemble of trajectories.

Let us denote the initial distance between two close trajectories $\delta(0)$, and their average distance at time $t$ and $t+$ $\Delta t$ as $\delta(t)$ and $\delta(t+\Delta t)$ respectively. Note that the classical algorithm for calculating the LLE $\left(\lambda_{\max }\right)$ is based on the assumption (13) and its estimation as $(\ln (\delta(t)-\delta(0))) / t$. Depending on $\delta(0)$, this property may not be true even for truly chaotic systems. To calculate the SDLE, we check whether the following inequality holds for a pair of vectors $(\overrightarrow{\mathrm{X}}(i), \overrightarrow{\mathrm{X}}(j))$ :

$\delta(k) \leq\|\overrightarrow{\mathrm{X}}(i)-\overrightarrow{\mathrm{X}}(j)\| \leq \delta(k)+\Delta \delta(k), \quad$ for $k=1,2,3, \ldots$, 


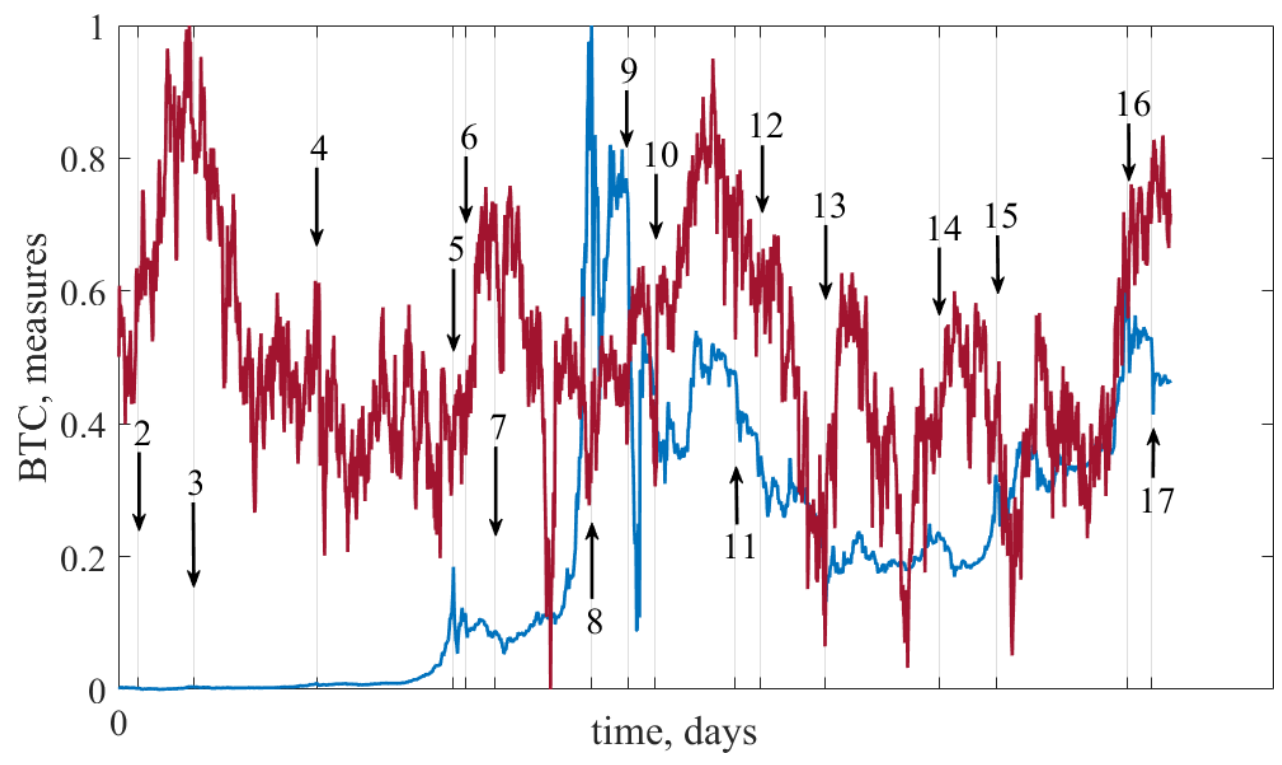

(a)

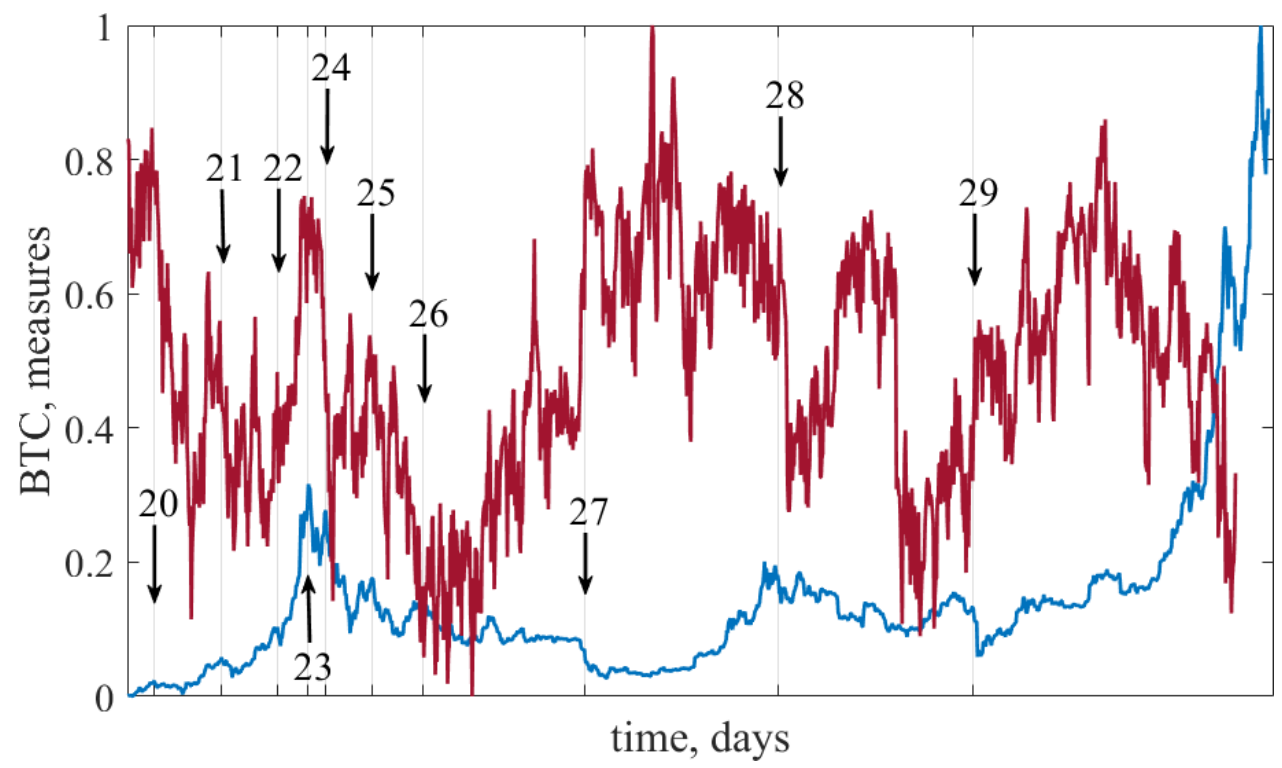

(b)

Figure 14: The dynamics of the LLE $\left(\lambda_{\max }\right)$ with Eckmann et al. method within the time window of the length 250 days and the time step of 1 day for the first (a) and second (b) periods. Exponents are calculated with $d_{E}=3$ and $d_{M}=2$

where $\delta(k)$ and $\Delta \delta(k)$ are arbitrarily chosen small values of distances, and

$$
\|\overrightarrow{\mathrm{X}}(i)-\overrightarrow{\mathrm{X}}(j)\|=\sqrt{\sum_{w=1}^{d_{E}}(x(i+(w-1) \tau)-x(j+(w-1) \tau))^{2}} .
$$

Geometrically, the last inequality defines a shell in high-dimensional space. Next, we investigate the dynamics of the same pairs of vectors $(\overrightarrow{\mathrm{X}}(i), \overrightarrow{\mathrm{X}}(j))$ in the middle of the shell and perform averaging over the ensemble by indices $i, j$. Since the exponential or power functions are of the greatest interest, we assume that logging and averaging can be reversed. Finally, the following equation looks like:

$$
\begin{array}{r}
\lambda(\delta(t))=\langle\ln \|\overrightarrow{\mathrm{X}}(i+t+\Delta t)-\overrightarrow{\mathrm{X}}(j+t+\Delta t)\|- \\
-\ln \|\overrightarrow{\mathrm{X}}(i+t)-\overrightarrow{\mathrm{X}}(j+t)\|\rangle / \Delta t,
\end{array}
$$

with the sampling intervals $t$ and $\Delta t$; the angle brackets correspond to the averaging over the indices $i, j$ inside the shell and

$$
\begin{array}{r}
\delta(t)=\|\overrightarrow{\mathrm{X}}(i+t)-\overrightarrow{\mathrm{X}}(j+t)\|= \\
=\sqrt{\sum_{w=1}^{d_{E}}(x(i+(w-1) \tau+t)-x(j+(w-1) \tau+t))^{2}} .
\end{array}
$$




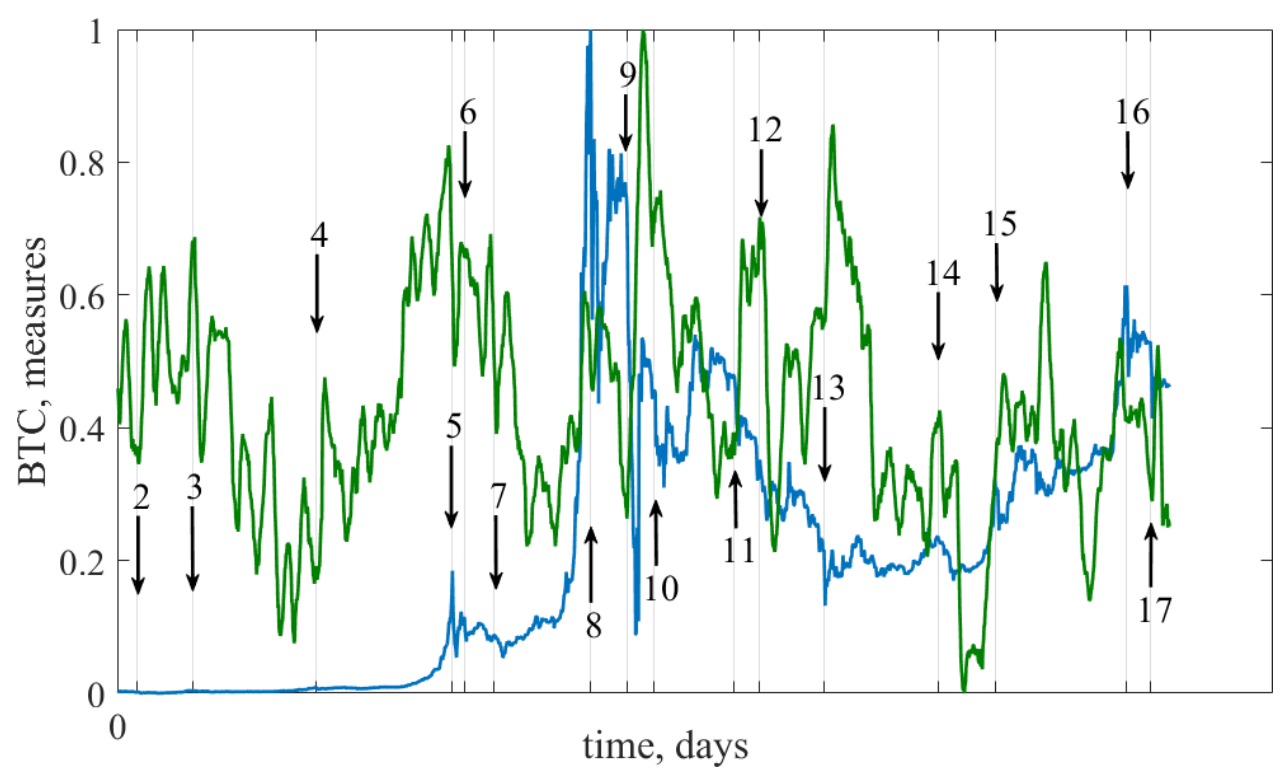

(a)

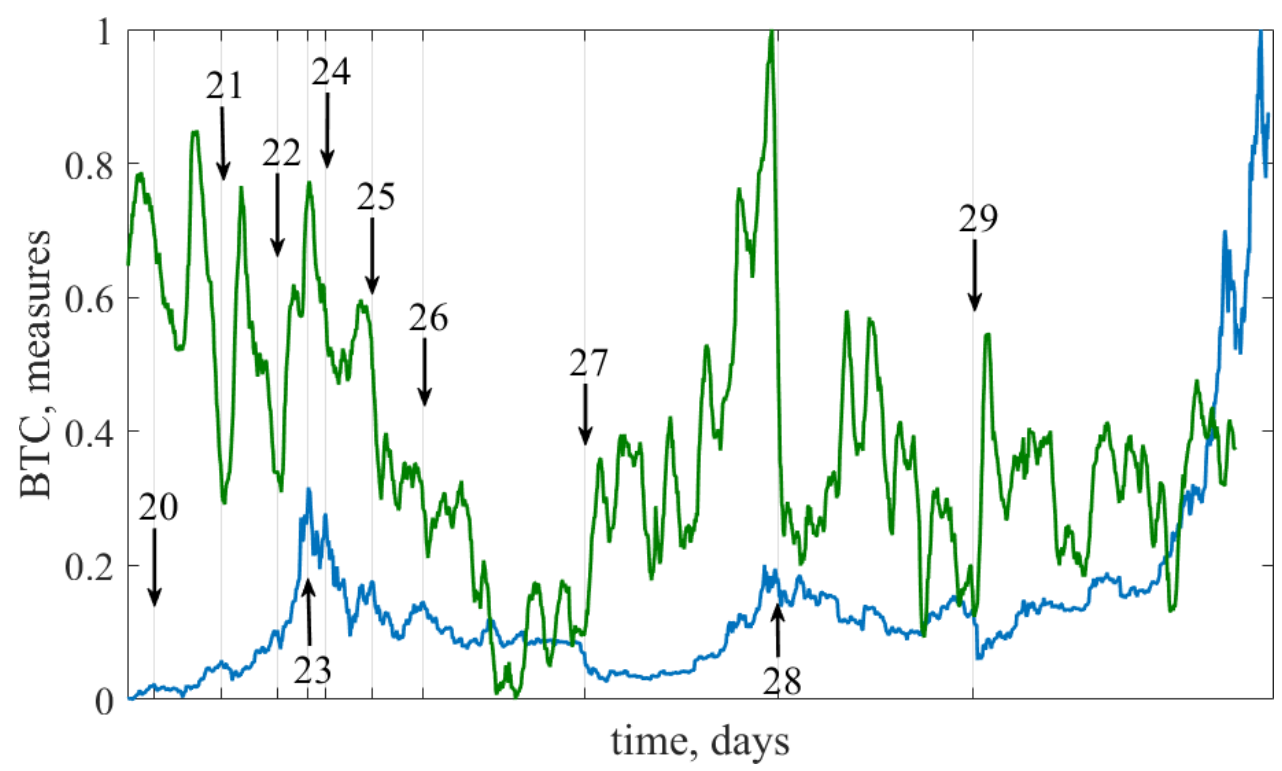

(b)

Figure 15: The dynamics of the LLE calculated with Rosenstein et. al. method within the time window length of 250 days and the time step of 1 day for first (a) and second (b) periods. Exponents are calculated with $d_{E}=3$ and $\tau=2$

Finally, note that

$$
I=\ln \delta(t)=\ln \delta(0)+\int_{0}^{t} \lambda(\delta(t)) d t
$$

Time series, characterizing economic systems of varying degrees of complexity, differ in magnitudes $\Delta \lambda=\lambda_{\max }-\lambda_{\min }, \Delta \delta=\delta_{\max }-\delta_{\min }$, and $I$. As an example, the integral measure $I$ is calculated for a sliding window of 400 days and step size of 1 day for the daily values of the BTC index (see figure 16).

Like the previous LLE indicators, SLDE is also a leading indicator. However, its disadvantage is the impossibility of accurate calculations for small window sizes which limits the possibilities of its usage.

\subsection{Lévy alpha-stable distribution}

Financial crises that regularly shake the world economy are characterized by noticeable fluctuations in stock indices, thereby causing noticeable changes in the statistical distributions of empirical data [111, 245].

In 1900, Bachelier proposed the first model for the stochastic process of returns - an uncorrelated random walk with independent, identically Gaussian distributed (i.i.d) random variables [246]. This model is natural if one considers the return over a time scale $\Delta t$ to be the result of many independent "shocks", which then lead by the central limit theorem to a Gaussian distribution of returns [246]. Some stylized facts of daily returns [247-249] re- 


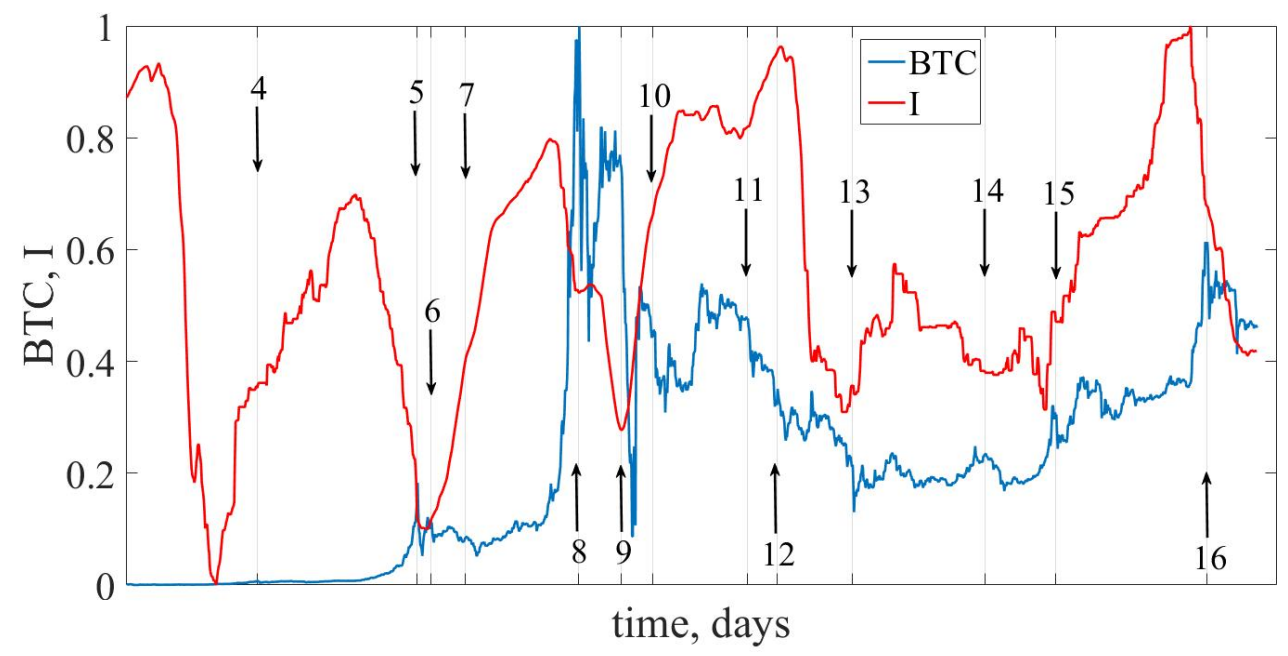

(a)

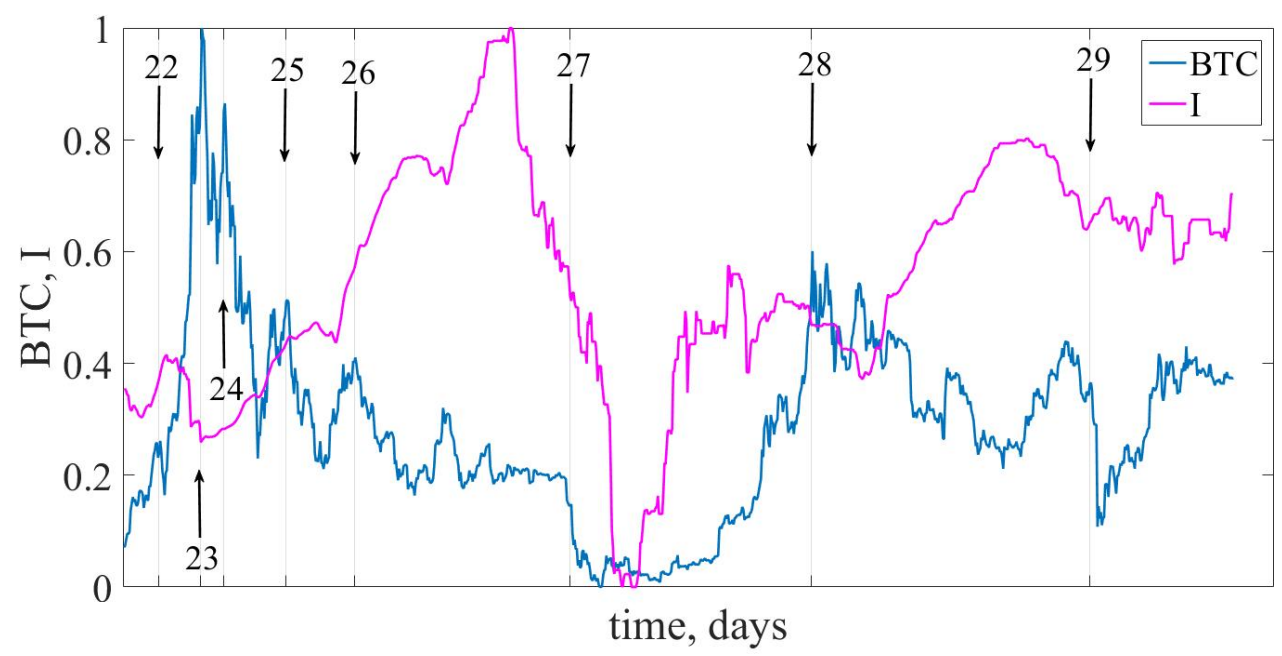

(b)

Figure 16: The dynamics of the SDLE exponents within the time window of length 400 days and the time step of 1 day for first (a) and second (b) periods

veal that distributions are leptokurtic and, therefore, Gaussian distribution does not fit well to the data. Sornette and Lux [250] pronounce that the distribution of such data may be not only leptokurtic, but it can also be characterized by fat tails [251-254]. Thus, it should belong to the class of fat-tailed distributions. Formally, it is said that they follow a power law. The emergence of power-law behavior in price fluctuations is argued to be a consequence of underlying complex mechanisms, such as feedback effects and correlations in financial markets [255-258]. Some theories associate this phenomenon with market impact and the distribution of large investors [247, 259], while other studies model the power-law behavior as a consequence of limited information and the true value of companies [260]. Such property is a symptom of self-organization and complexity which are prominent for economic systems. In Chakraborty et al. [261] paper it was established that currencies of several frontiers that are outside of inverse cubic law (with an exponent of $\alpha \simeq 3$ ) belong to the Levy-stable regime and are expected to be yet emerging and having sudden large changes such as crashes and critical events, while those of most developed exhibited inverse cubic law.

Recently, it has been reported that Bitcoin is becoming more mature, following inverse cubic law [262-264]. Besides, Stjepan Begušić et al. [265], motivated by the rise of novel assets based on blockchain technology, presented a detailed analysis of trade-level data of the BTC/USD pairs from five large Bitcoin exchanges: Mt. Gox, BTC-e, Bitstamp, Bitfinex, and Kraken. They applied two estimation methods and a resampling-based technique to statistically validate if the main cryptocurrency follows powerlaw behavior or not. Their study presented that the exponent $\alpha$ lie within the range $2<\alpha<2.5$ that gives the evidence that the cryptocurrencies market is much more volatile than the stock market, and that Bitcoin returns exhibit much heavier tails. Moreover, they find that such a phenomenon is universal as such behavior holds across multiple exchanges and tiny intervals. Their results imply 
that Bitcoin lies outside of the Levy-stable region which provides the existence of a finite second moment, and a basis for the usage of standard financial theories for portfolio optimization and risk management.

In figure 17 the daily returns of Bitcoin and contrast it with a sequence of i.i.d. Gaussian random variables are presented.

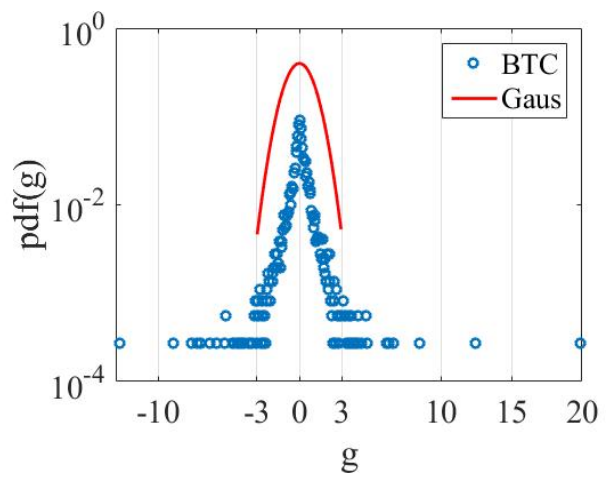

(a)

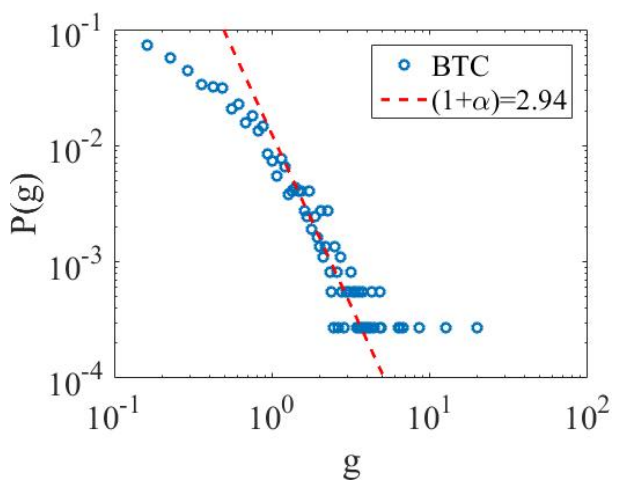

(b)

Figure 17: Probability density function of BTC daily normalized returns for the whole period (a). Cumulative distribution of the normalized BTC daily returns (b). Fits yield values $\alpha=1.94 \pm 0.02$

It is obvious that the distribution of returns has heavy tails and in general case can be described as

$$
P(g>x) \sim x^{-(1+\alpha)}, \quad \alpha \in(0,2] .
$$

Figure $17 \mathrm{~b}$ it can be seen that Bitcoin exhibits the inverse cubic law.

In the analysis of cotton prices, Mandelbrot observed that in addition to being non-Gaussian, the process of returns shows another interesting property: "time scaling" that is, the distributions of returns for various choices of $\Delta t$, ranging from 1 day up to 1 month have similar functional forms [266]. Motivated by (i) pronounced tails, and (ii) a stable functional form for different time scales, Mandelbrot proposed that the distribution of returns is consistent with a Levy stable distribution [266-268] - that is, the returns can be modeled as a Levy stable process. Levy stable distributions arise from the generalization of the Cen- tral Limit Theorem (CLT) to random variables that do not have a finite second moment.

The CLT [269], which offers the fundamental justification for approximate normality, points to the importance of alpha-stable distribution: they are the only limiting laws of normalized sums of independent, identically distributed random variables. Gaussian distributions, the best-known member of the stable family, have long been well understood and widely used in all sorts of problems. However, they do not allow for large fluctuations and are thus inadequate for modeling high variability. Non-Gaussian stable models, on the other hand, do not share such limitations. In general, the upper and lower tails of their distributions decrease like a power function. In literature, this is often characterized as heavy or long tails. In the last two or three decades, data that seem to fit the stable model has been collected in fields as diverse as economics, telecommunications, hydrology, and physics [248].

Consequently, a probability model with a power tail can be suitable for identifying processes with extreme events. It was discovered that alpha-stable distributions fit better than the Gaussian distribution to financial and spot markets. It is still debatable whether Lévy stable distribution is applicable since there is not enough theoretical material, and there is not a universal analyzing method for estimating parameters of Lévy stable distribution.

\subsubsection{Lévy's stable distribution properties}

Lévy stable distribution being the generalization of the CLT, became an addition to a wide class of distributions. The term stable is such characteristic of distribution where the shape (up to scale and shift) retains under addition: if $X, X_{1}, X_{2}, \ldots, X_{n}$ are independent, identically distributed random variables, then for every $n$

$$
X_{1}+X_{2}+\cdots+X_{n} \stackrel{d}{=} c_{n} X+d_{n}
$$

for some constants $c_{n}>0$ and $d_{n} \in \mathbb{R}$, where $X_{1}, \ldots, X_{n}$ are independent, identical copies of $X$.

The class of all laws that satisfy condition (20) is presented by 4 parameters: $\alpha \in(0,2]$ is the index of stability or characteristic exponent where a smaller value of $\alpha$ corresponds to more severe tails of the distribution. The parameter $\beta \in[-1,1]$ is called the skewness parameter of the law. If $\beta=0$, the distribution is symmetric. In the case when $\beta>0$, it is skewed toward the right, otherwise to the left. The last two parameters stand for the scale $\gamma \in[0, \infty)$ and $\delta \in(-\infty, \infty)$ the location parameters of the distribution. Since random variables $X$ is characterized by four parameters, we will denote $\alpha$-stable distribution by $S(\alpha, \beta, \gamma, \delta)$ and write

$$
X \sim S(\alpha, \beta, \gamma, \delta)
$$

Lévy stable distributions cannot be defined in closed form expression except few cases: the case of $(\alpha, \beta)=$ $(2,0)$ corresponds to the Gaussian distribution, $(\alpha, \beta)=$ $(1,0)$ to the Cauchy distribution. Instead, it is expressed 
in terms of their Fourier transforms or characteristic functions (CF). If the density $f(x)$ exists, CF of that density can be expressed as

$$
\lambda(k)=E \exp (i k X)=\int_{-\infty}^{\infty} \exp (i k x) f(x) d x,
$$

where $k$ denotes the Fourier transformed variable. Thus, the inverse Fourier transform

$$
f(k)=\frac{1}{2 \pi} \int_{-\infty}^{\infty} \exp (-i k x) \lambda(k) d k
$$

allows us to reconstruct probability density function with a known characteristic function.

As we do not have any analytical expression for the probability density of a random variable $X_{i}$, for Lévy stable distribution, if the variable $x_{i}$ follows $S(\alpha, \beta, \gamma, \delta)$, the $\mathrm{CF}$ can be expressed as [270]:

$$
\lambda(k)= \begin{cases}\exp i \delta k-\gamma^{\alpha}|k|^{\alpha}\left[1-i \beta \operatorname{sgn}(k) \tan \left(\frac{\pi \alpha}{2}\right)\right], & (\alpha \neq 1), \\ \exp i \delta k-\gamma|k|\left[1+i \beta \operatorname{sgn}(k) \frac{2}{\pi} \ln |k|\right], & (\alpha=1) .\end{cases}
$$

\subsubsection{Methods for estimation of stable law parameters}

There are numerous approaches that can estimate stable distribution parameters. Since the probability density functions are not always expressed in a closed form, there are some challenges to overcome the analytic difficulties. Thus, there have been constructed a variety of methods: the approximate maximum likelihood (ML) estimation [271, 272], quantiles method [273, 274], fractional lower order moment method [275, 276], method of log-cumulant [277], the logarithmic moment method [278] and more. Unfortunately, some of those methods cannot be applied due to computational problems associated with a limited range of estimation, restricted range of parameters, high computational costs, or requiring a large number of data. However, several of them should be mentioned.

\subsubsection{Maximum likelihood method}

DuMouchel was the first to obtain approximate ML estimates of $\alpha$ and $\gamma$ (assuming $\delta=0$ ) [279]. A multinomial approximation to the likelihood function is used in his approach. Under some additional assumptions on $\hat{\alpha}$ and the likelihood function, DuMouchel has shown the obtained estimates to be consistent and asymptotically normal. However, the computational effort involved seems considerable.

A direct method can be formulated, after Brorsen and Yang [271], as follows. The standard symmetric probability density functions defined by Zolotariev [280] is presented as:

$$
\begin{array}{r}
f_{\alpha}(x)=\frac{\alpha}{\pi|1-\alpha|} x^{1 /(\alpha-1)} \times \\
\times \int_{0}^{\pi / 2} U_{\alpha}(\eta, 0) \exp -x^{\alpha /(\alpha-1)} U(\eta, 0) d \eta,
\end{array}
$$

for $\alpha \neq 1, x>0$, where $U_{\alpha}$ is defined by:

$$
U_{\alpha}\left(\eta, \eta_{0}\right)=\left(\frac{\sin \alpha\left(\eta-\eta_{0}\right)}{\cos \eta}\right)^{\alpha /(1-\alpha)}\left(\frac{\cos \eta-\alpha\left(\eta-\eta_{0}\right)}{\cos \eta}\right)
$$

and $\eta_{0}$ is explained here [281]. Therefore, the parameters $\alpha, \gamma$, and $\delta$ can be estimated from the observations $\left\{x_{i} \mid i=\right.$ $1,2, \ldots, N\}$ by maximizing the log likelihood function:

$$
\begin{array}{r}
\sum_{i=1}^{N} \log f_{\alpha}\left(z_{i}\right)=n \log \alpha-n \log (\alpha-1)+ \\
+\sum_{i=1}^{N} \frac{\log z_{i}}{\alpha-1}+ \\
+\sum_{i=1}^{N} \log \int_{0}^{\pi / 2} U_{\alpha}(\eta, 0) \exp -z_{i}^{\alpha /(\alpha-1)} U_{\alpha}(\eta, 0) d \eta,
\end{array}
$$

where $z_{i}=\left|x_{i}-\delta\right| / \gamma$.

To avoid the discontinuity and non-differentiability of the symmetric $\alpha$-stable density function at $\alpha=1$, alpha is restricted to be greater than one. Caution must be used when evaluating the integrals in equations (23) and (25), since the integrals are singular at $\eta=0$.

An obvious disadvantage of this method is that it is a highly nonlinear optimization problem and no initialization and convergence analysis is available.

\subsubsection{Quantiles methods}

This method is focused on empirical quantiles, which has been introduced by Fama and Roll, with the assumptions that $\alpha>1, \beta=0$, and $\delta=0$ [273]. However, it was much more appreciated through McCulloch [274] after its extension to include asymmetric distribution for $\alpha \in[0.6,2]$.

In order to quantify the four parameters of the stable distribution, we consider $N$ independent variables $x_{i}$ that follow alpha-stable distribution (21) [274, 282]. Then, we need to define five empirical quantiles of probability levels $5 \%, 25 \%, 50 \%, 75 \%$, and $95 \%$. Then we have to obtain two intermediate quantities:

$$
\begin{cases}\hat{v}_{\alpha}=\frac{\hat{Q}_{0.95}-\hat{Q}_{0.05},}{\hat{Q}_{0.75}-\hat{Q}_{0.25},} & \hat{v}_{\beta}=\frac{\hat{Q}_{0.95}+\hat{Q}_{0.05}-2 \hat{Q}_{0.05}}{\hat{Q}_{0.95}-\hat{Q}_{0.05}}, \\ \hat{\alpha}=\Psi_{1}\left(\hat{v}_{\alpha}, \hat{v}_{\beta}\right), & \hat{\beta}=\Psi_{2}\left(\hat{v}_{\alpha}, \hat{v}_{\beta}\right),\end{cases}
$$

where $\hat{Q}_{p}(p=0.05,0.25,0.5,0.75,0.95)$ is the corresponding sample data with which quantile is calculated, and $\Psi_{1}$ with $\Psi$ are the interpolating functions the values of which can be found in Table I-IV by McCulloch [274]. Further, the scale parameter is given by:

$$
\hat{\gamma}=\frac{\hat{Q}_{0.75}-\hat{Q}_{0.25}}{\Psi_{3}(\hat{\alpha}, \hat{\beta})},
$$

where $\mathrm{Psi}_{3}(\hat{\alpha}, \hat{\beta})$ is given in Table V [274]. For simplicity of the location parameter, we can define the variable which is predefined in the following form:

$$
\xi= \begin{cases}\delta+\beta \gamma \tan \frac{\pi \alpha}{2}, & \text { if } \alpha \neq 1, \\ \delta, & \text { if } \alpha=1 .\end{cases}
$$


In consistence with the corresponding parameter $\xi$ which can be estimated by $\hat{\xi}=\hat{Q}_{0.5}+\hat{\gamma} \Psi_{5}(\hat{\alpha, \beta})\left(\Psi_{5}\right.$ can be obtained through linear interpolation according to Table VII [274]), the location parameter $\delta$ is given by:

$$
\hat{\delta}=\hat{\xi}+\hat{\beta} \hat{\gamma} \tan \frac{\pi \hat{\alpha}}{2} .
$$

\subsubsection{Empirical characteristic function method}

Analyzing data, we often assume that they are ergodic [283]. In general, if random variables are ergodic with the integrable function $f(x)$, and the measure $\rho(x) d x$ in the space $M$, then the following equation holds [284]:

$$
\lim _{N \rightarrow \infty} \frac{1}{N} \sum_{i=1}^{N} \exp \left(i k x_{i}\right)=\int_{-\infty}^{\infty} \exp (i k x) \rho(x) d x .
$$

Then, to consider characteristic functions, equation (27) comes out to be the following ergodic equality [284]:

$$
\lim _{N \rightarrow \infty} \frac{1}{N} \sum_{i=1}^{N} \exp \left(i k x_{i}\right)=\int_{-\infty}^{\infty} \exp (i k x) f(x) d x
$$

for which we have

$$
\hat{\lambda}(k)=\lim _{N \rightarrow \infty} \frac{1}{N} \sum_{i=1}^{N} \exp \left(i k x_{i}\right) .
$$

This assumption allows us to empirically obtain the probability distribution. Hence, the empirical characteristic function $\lambda_{N}(k)$ can be calculated as

$$
\hat{\lambda}_{N}(k)=\frac{1}{N} \sum_{i=1}^{N} \exp \left(i k x_{i}\right)
$$

Then, according to Koutrouvelis' [270, 285] regression type from (22) it can be derived that

$$
\log \left(-\log \left(|\lambda(k)|^{2}\right)\right)=\log \left(2 \gamma^{\alpha}\right)+\alpha \log (k) .
$$

The imaginary and real parts of $\lambda(k)$ are given by

$$
\left\{\begin{array}{l}
\lambda_{\mathrm{I}}(k)=\exp \left(-|\gamma k|^{\alpha}\right) \cdot i \sin \left[\delta k-|\gamma k|^{\alpha} \beta \operatorname{sgn}(k) \omega(k, \alpha)\right], \\
\lambda_{\mathrm{R}}(k)=\exp \left(-|\gamma k|^{\alpha}\right) \cdot \cos \left[\delta k-|\gamma k|^{\alpha} \beta \operatorname{sgn}(k) \omega(k, \alpha)\right],
\end{array}\right.
$$

where

$$
\omega(k, \alpha)= \begin{cases}\tan \frac{\pi \alpha}{2}, & \alpha \neq 1, \\ \frac{2}{\pi} \ln |k|, & \alpha=1 .\end{cases}
$$

Suppose $\Upsilon:=\arctan \left(\lambda_{\mathrm{I}}(k) / \lambda_{\mathrm{R}}(k)\right)$. Then, in the condition $\alpha \neq 1$, the last two equations lead, apart from considerations of principal values, to

$$
\Upsilon(k)=\delta k-\beta \gamma^{\alpha} \tan \left(\frac{\pi \alpha}{2}\right) \operatorname{sgn}(k)|k|^{\alpha} .
$$

Equation (31) depends only on $\alpha$ and $\gamma$, and it suggests that we estimate these parameters by regressing

$$
y=\log \left(-\log \left|\lambda_{N}(k)\right|^{2}\right)
$$

on $\omega=\log (k)$ in the model

$$
y_{l}=m+\alpha \theta_{l}+\epsilon_{l}, \quad \text { for } l=1, \ldots, L,
$$

where $y_{l}=\log \left(-\log \left(\hat{\lambda}\left(k_{l}\right)\right)^{2}\right), m=\log \left(2 \gamma^{\alpha}\right), \theta_{l}=\log \left(k_{l}\right)$, and $\epsilon_{l}$ responds for an error term. The proposed real data set for $L$ (see Koutrouvelis [270], Table I) is given by $k_{l}=$ $\pi l / 25(l=1, \ldots, L)$.

With estimated and fixed parameters $\alpha$ and $\gamma$, the symmetric parameter $\beta$ and location parameter $\delta$ can be obtained by linear regression estimation

$$
\begin{array}{r}
z_{q}=\delta k_{q}-\beta \gamma^{\alpha} \tan \left(\frac{\pi \alpha}{2}\right) \operatorname{sgn}\left(k_{q}\right)\left|k_{q}\right|^{\alpha}+v_{q}, \\
\text { for } q=1, \ldots, Q,
\end{array}
$$

where $z_{q}=\Upsilon_{N}\left(k_{q}\right)+\pi l_{N}\left(k_{q}\right), v_{l}$ denotes an error term, and the proposed real data set for $Q$ (see Koutrouvelis [270], Table II) is $k_{q}=\pi q / 50(q=1, \ldots, Q)$.

\subsubsection{Related studies and corresponding results}

Recently, the use of dynamic indicators, precursors of crashes in stock markets using the parameters of a $\alpha$-stable distribution was proposed by us in the papers [76, 77, 286] and later repeated in a recent paper [30]. From the data above, we estimate the parameters $\alpha$ and $\beta$ of the stable distribution that the best describes the empirical returns. Figure 18 shows the dynamics of the parameter $\alpha$ as a more informative indicator.

From the figure 18 we can see that our parameters start to decrease in crisis states. Such abnormal behavior can serve as an indicator or precursor of crashes and critical states.

\section{Recurrence analysis}

In 1890 the mathematical foundations of recurrence were introduced by Henri Poincaré, resulting in the Poincaré recurrence theorem [287]. This theorem states that certain systems will return to their arbitrarily close, or exactly the same initial states after a sufficiently long but finite time. Such property in the case of deterministic behavior of the system allows us to make conclusions regarding its future development.

\subsection{Recurrence plot}

Recurrence plot (RP) have been introduced to study dynamics and recurrence states of complex systems. When we create RP, at first, from recorded time series we reconstruct phase-space trajectory. Then, according to Eckmann et al. [231], we consider a trajectory $\vec{X}(i)$ on the reconstructed trajectory. The recurrence plot is an array of dots in a $N \times N$ matrix, where dot is placed at $(i, j)$ whenever $\vec{X}(j)$ is sufficiently close to $\vec{X}(i)$, and both axes are time axes which mathematically can be expressed as

$$
R_{i j}=\mathcal{H}(\epsilon-\|\overrightarrow{\mathrm{X}}(i)-\overrightarrow{\mathrm{X}}(j)\|), \quad \text { for } i, j=1, \ldots, N,
$$




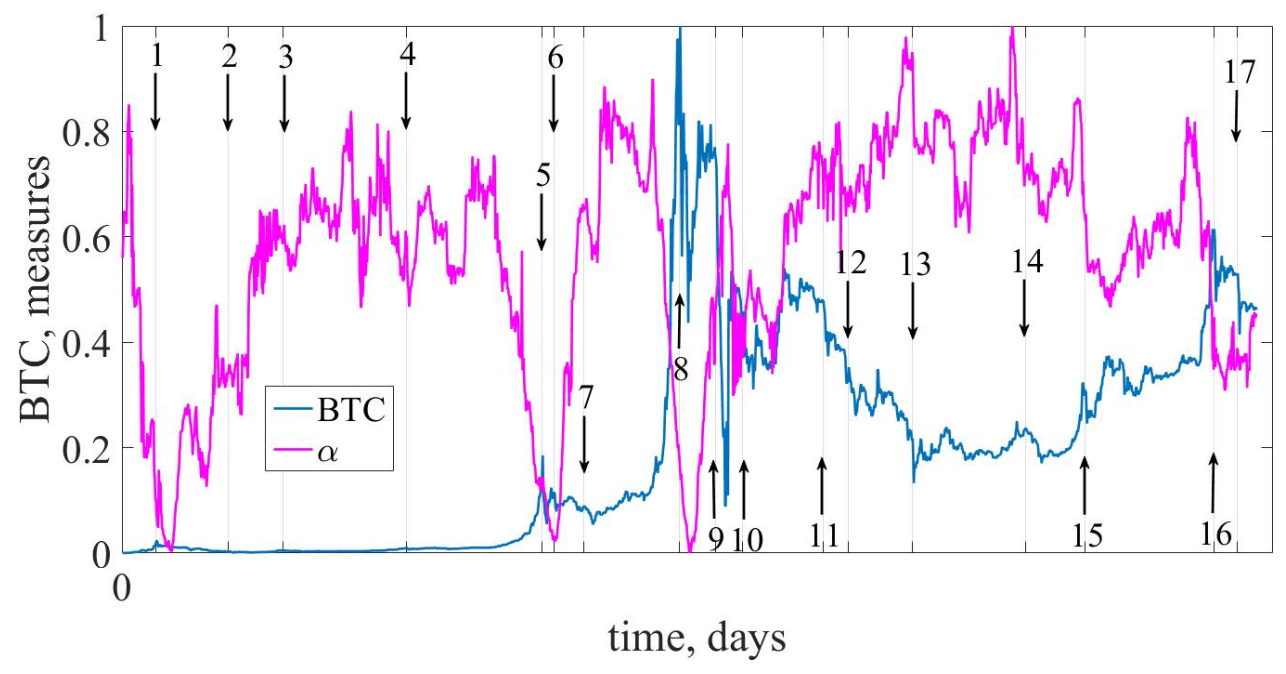

(a)

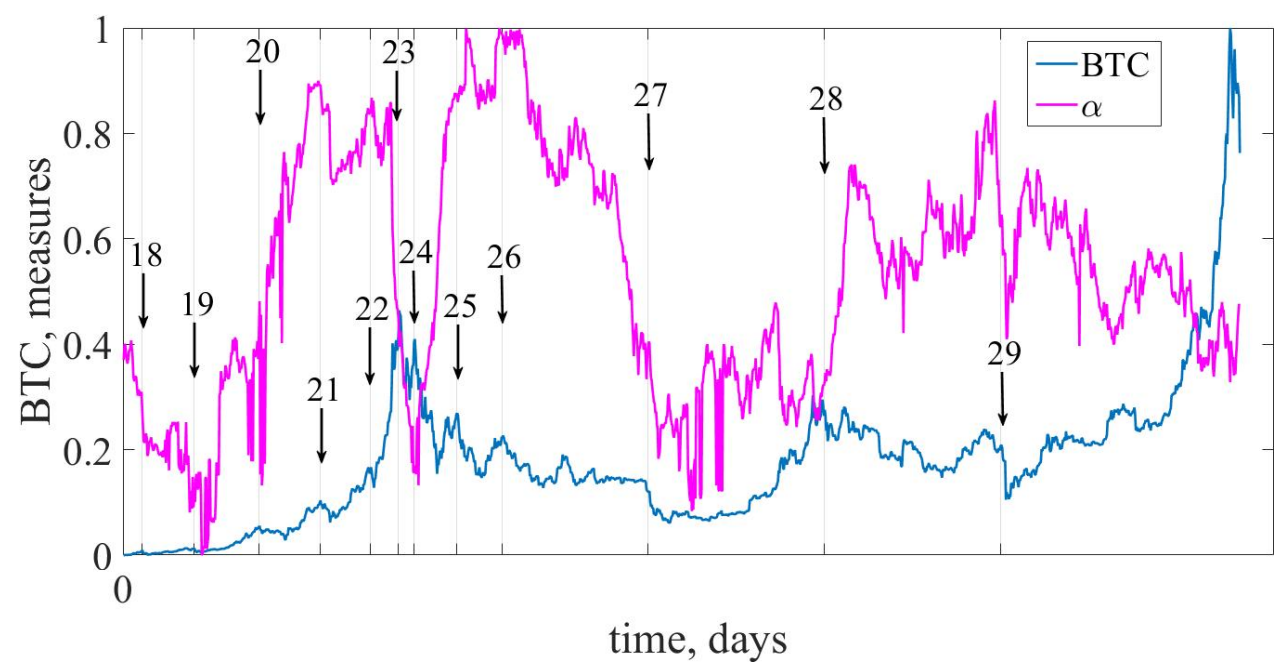

(b)

Figure 18: The Bitcoin time series and estimated for them parameter $\alpha$. Vertical arrows indicate crashes and critical events

where $\|$ II is a norm (representing the spatial distance between the states at times $i$ and $j$ ); $\epsilon$ is a predefined recurrence threshold, and $\mathcal{H}$ is the Heaviside function. As a result, the matrix captures a total of $N^{2}$ binary similarity values. A synthetic example is presented in figure 19.

Typically an $L_{p}$-norm is applied to determine the pairwise similarity between two vectors. Accordingly to Webber and Zbilut [139], there are such candidates that can serve as a distance measure:

- the $L_{1}$-norm (Manhattan distance);

- the $L_{2}$-norm (Manhattan distance);

- the $L_{\infty}$-norm (Manhattan distance);

In accordance with our results, Maximum distance seems to be a suitable choice. It is often used as it is independent of the phase space dimension, easy to calculate, and allows some analytical expression [288-290].

Also, as it can be seen from equation (36), the similarity between vectors is determined by a threshold $\epsilon$. The choice of $\epsilon>0$ ensures that all vectors that lie within this radius are similar to each other, and that dissimilarity up to a certain error is permitted [287].

The fixed radius for recurrent states is the commonly used condition, which leads to equally sized $\epsilon$ neighborhoods. The shape in which neighborhoods lie is determined by the distance metric. Applying the fixed threshold with the distance metric, we define recurrence matrices that are symmetric along the middle diagonal. The self-similarity of the multi-dimensional vectors reflects in the middle diagonal which is commonly referred to as line of identity (LOI). In contrast, it is not guaranteed that a recurrence matrix is symmetric, if the condition of fixed number of nearest neighbors is applied. For specific purposes (e.g., quantification of recurrences), it can be useful to exclude the LOI from the RP, as the trivial recurrence of a state with itself might not be of interest [291]. 


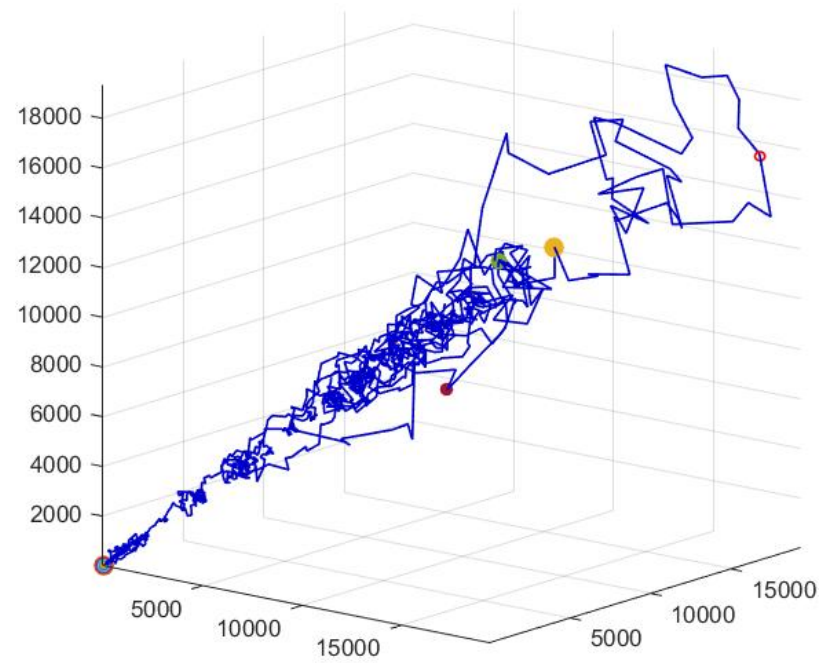

(a)

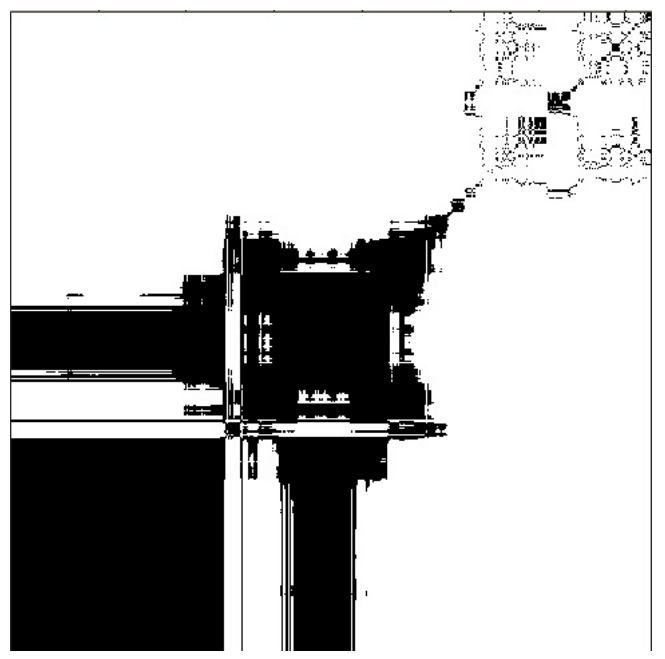

(b)

Figure 19: Phase portrait (a) and corresponding RP (b) for BTC

\subsubsection{Recurrence plots and their structures}

The visualization of trajectories and hidden patterns of the systems is the "destiny" of RP [291, 292].

The dots within RP, representing the time evolution of the trajectories, exhibit characteristic large-scale and small-scale patterns. Large-scale patterns of RP can be classified as homogeneous, periodic, drift, and disrupted [289, 293, 294]:

- Homogeneous typify behavior of autonomous and stationary systems, which consist of a large number of recurrence points that are homogeneously distributed (relaxation times are short).

- Periodic represents long, uninterrupted, and diagonally oriented structures that represent which indicate periodic behavior. These lines are usually distributed regularly.
- Paling or darkening from the LOI to the outer corners in $\mathrm{RP}$ is characteristic of drift.

- Disrupted distribution of recurrence points may serve as an indicator of drastic changes as well as extreme events in the system dynamics. In these cases, RP can be used to find and assess extreme and rare events by scoring the frequency of their repeats.

\subsubsection{Recurrence quantification analysis}

For a qualitative description of the system, the graphic representation of the system suits perfectly. However, the main disadvantage of graphical representation is that it forces users to subjectively intuit and interpret patterns and structures presented within the recurrence plot. Also, with plots' increasing size, they can be hardly depicted on graphical display as a whole. As a result, we need to work with separated parts of the original plot. Analysis in such a way may create new defects that which should distort objectivity of the observed patterns and lead to incorrect interpretations. To overcome such limitation and spread an objective assessment among observers, in the early 1990s by Webber and Zbilut were introduced definitions and procedures to quantify RP's complexity, and later, it has been extended by Marwan et al. [294-296].

The small-scale clusters can represent a combination of isolated dots (chance recurrences). Similar evolution at different periods in time or in reverse temporal order will present diagonal lines (deterministic structures) as well as vertical/horizontal lines to inscribe laminar states (intermittency) or systems that paused at singularities. For the quantitative description of the system, such small-scale clusters serve the base of the recurrence quantification analysis (RQA).

Usually, first acquaintance with classical RQA starts with recurrence point density, or, as it is known, recurrence rate $(R R)$ :

$$
R R=\frac{1}{N^{2}} \sum_{i, j=1}^{N} R_{i, j}
$$

It enumerates the probability that any state of the system will recur. It is the simplest measure that is computed by taking the number of nearest points forming short, spanning row and columns of the recurrent plot. It summarizes them and divides by the number of possible points in the recurrence matrix of size $N^{2}$.

The remaining measure relies on the frequency distribution of line structures in the RP. First, we consider the histogram of the length of the diagonal structures in the RP

$$
P(l)=\sum_{i, j=1}^{N}\left\{\left(1-R_{i-1, j-1}\right) \cdot\left(1-R_{i+l, j+l}\right) \cdot \prod_{k=0}^{l-1} R_{i+k, j+k}\right\} .
$$

The fraction recurrence points on the recurrence plots that form line segments of minimal length $\mu$ parallel to the matrix diagonal is the measure of determinism (DET):

$$
D E T^{(\mu)}=\frac{\sum_{l=\mu}^{N} l \cdot P(l)}{\sum_{i, j=1}^{N} R_{i, j}}=\frac{\sum_{l=\mu}^{N} l \cdot P(l)}{\sum_{l=1}^{N} l \cdot P(l)} .
$$


Systems that exhibit deterministic dynamics are mainly characterized by diagonal lines. Long diagonal lines indicate periodic signals, but short diagonal lines stand for chaotic behavior. Regarding the quantitative analysis, typically, only the lines with minimal length $\mu=2$ are considered. If $\mu=1$, then DET and RR are identical. For some systems, DET becomes more reliable if $\mu>2$. Here, $\mu$ serves as a filter, excluding the shorter lines. However, it should be noted that too large $\mu$ may spoil the histogram $P(l)$ and thus the reliability of DET.

The results of calculations of window dynamics of the considered recurrence measures are presented in Figure 20. RR and DET are calculated for local time series of 50 days and a step of 1 day. In this case, the beginning of a crash or critical event is at point 100 .

It is evident that the two recurrent measures during abnormal periods decrease long before the actual anomaly. The complex system becomes less recurrent and less deterministic which is logical in the periods approaching critical phenomena. And, consequently, RR and DET can be used as precursors of critical and crash phenomena.

\subsubsection{Chaos recurrent measures}

The corresponding measure of entropy is related to the recurrence properties that may be peculiar for the nonlinear complex system and important class of recurrence quantifiers are those that try to capture the level of complexity of a signal [78, 84, 297]. In accordance with this study, the entropy diagonal line histogram (DLEn) is of the greatest interest which uses the Shannon entropy of the distribution of diagonal lines $P(l)$ to determine the complexity of the diagonal structures within the recurrence plot. One of the most know quantitative indicators of the recurrence analysis can be defined as:

$$
D L E n=-\sum_{l=l_{\min }}^{l=l_{\max }} p(l) \ln p(l)
$$

and

$$
p(l)=\frac{P(l)}{\sum_{l=l_{\min }}^{N} P(l)},
$$

where $p(l)$ captures the probability that a diagonal line has exactly length $l$, and DLEn reflects the complexity of deterministic structure in the system. Further calculations were provided and presented in figure 21 for both Bitcoin time series.

However, as follows from the analysis of the entropy indicators, the results may differ for different data preparation. Further, we take into account two types of Shannon entropy-based approaches: recurrence period density entropy (RPDEn) and recurrence entropy (RecEn).

The RPDEn is the quantitative measure of the recurrence analysis that is useful for characterizing the periodicity or absolutely random processes in the time series. It is useful for quantifying the degree of repetitiveness [298, 299]. Considering embedded data point $\vec{X}(i)$ from the phase space and suitable threshold $\epsilon$ in $d_{E}$-dimensional space. Then the trajectory is followed forward in time until it has left the corresponding threshold $\epsilon$. Subsequently, the time $j$ at which the trajectory first returns to this ball and the period $T$ of previous and current states is recorded. The procedure is repeated for all states of the RPs, forming a histogram of recurrence times $R(T)$. The histogram is then normalized to give the recurrence time probability density:

$$
P\left(T_{i}\right)=\frac{R\left(T_{i}\right)}{\sum_{i=1}^{T_{\max }} R\left(T_{i}\right)},
$$

where $T_{\max }=\max \left\{T_{i}\right\}$. The normalized entropy of the obtained density can be defined as:

$$
\text { RPDEn }=\frac{-\sum_{i=1}^{T_{\max }} P\left(T_{i}\right) \ln P\left(T_{i}\right)}{\ln T_{\max }} .
$$

In fact, based on the length of the sequences of neighboring points in the phase space: the more points are neighborhoods, the lower the value of the entropy according to equation (37). The comparing of RPDEn and Bitcoin's critical states can be seen in figure 22 .

However, recent articles [297, 300] present a slightly different technique for calculating recurrent entropy using a novel way to extract information from the recurrence matrix. To properly define it, we need to define the microstates $F(\epsilon)$ for the RP that are associated with features of the dynamics of the time series. Selecting the appropriate metric and using the Heaviside function, we evaluate the matrices of dimensions $N \times N$ that are sampled from the RP. The total number of microstates for a given $N$ is $N_{m s}=2^{N^{2}}$. The microstates are populated by $\bar{N}$ random samples obtained from the recurrence matrix such that:

$$
\bar{N}=\sum_{i=1}^{N_{m s}} n_{i}
$$

with $n_{i}$ representing the number of times that a microstate $i$ is observed. The probability of occurrence of the related microstate $i$ can be obtained as:

$$
p_{i}=n_{i} \cdot(\bar{N})^{-1} \text {. }
$$

The RecEn of the RP associated with the probability distribution of the corresponding microstates is given by the following equation:

$$
\operatorname{RecEn}=\sum_{i=1}^{N_{m s}} p_{i} \ln p_{i} .
$$

In figure 23 we can see the performance of RecEn accordingly to the described above method.

A vertical line of length $l$ starting from a dot $(i, j)$ means that the trajectory starting from $\vec{X}(j)$ remains close to $\vec{X}(i)$ during $l-1$ time steps. A diagonal black line of length $l$ starting from a dot $i, j$ means that trajectories starting from $\vec{X}(i)$ and $\vec{X}(j)$ remain close during $l-1$ time steps, thus these lines are related to the divergence of the trajectory segments. The average diagonal line length

$$
L_{\text {mean }}=\frac{\sum_{l=l_{\min }}^{N} l \cdot P(l)}{\sum_{l=l_{\text {min }}}^{N} P(l)}
$$

is the average time that two segments of the trajectory are close to each other and can be interpreted as the mean prediction time. Here, $P(l)$ is a histogram of diagonal lines of length $l$. 


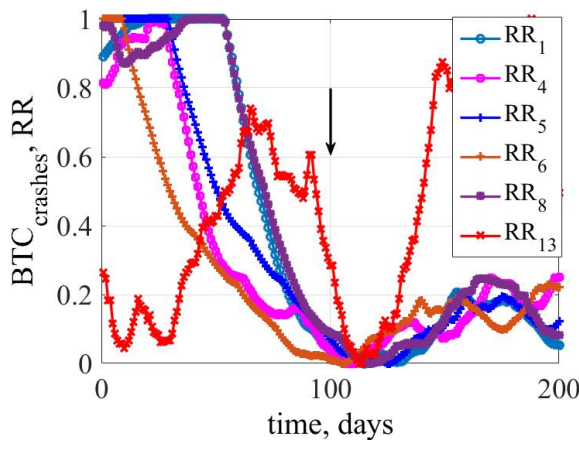

(a)

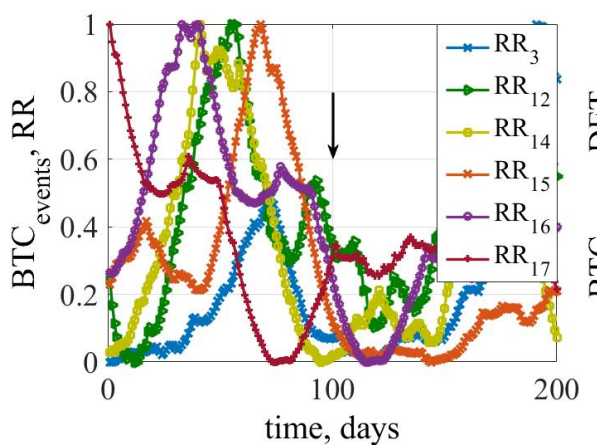

(c)

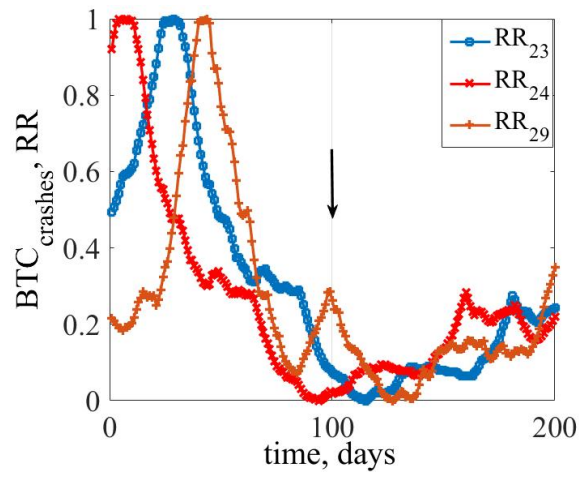

(e)

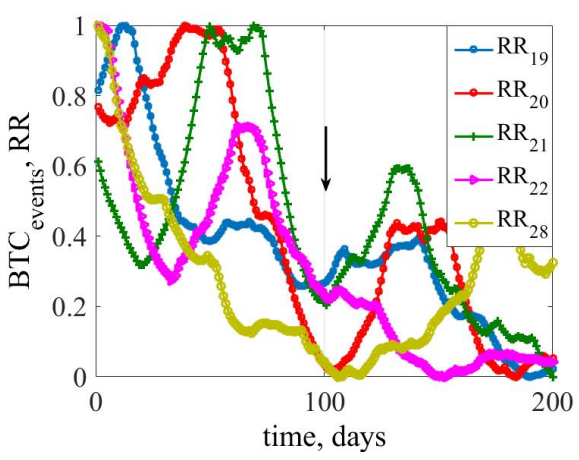

(g)

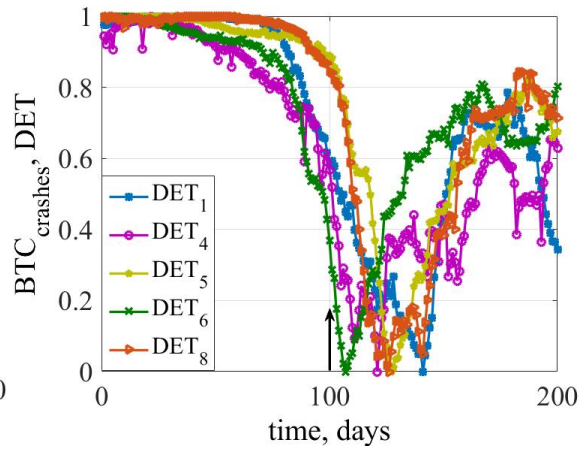

(b)

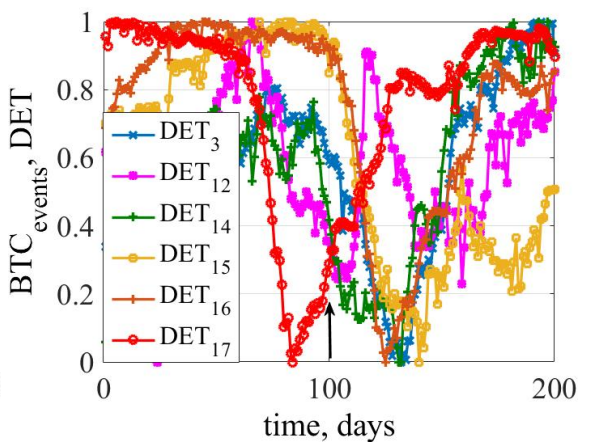

(d)

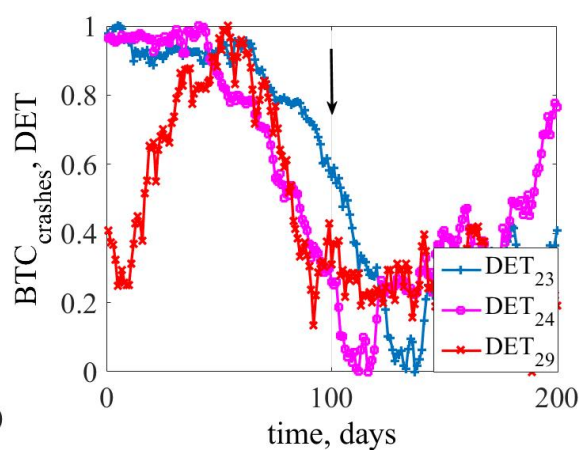

(f)

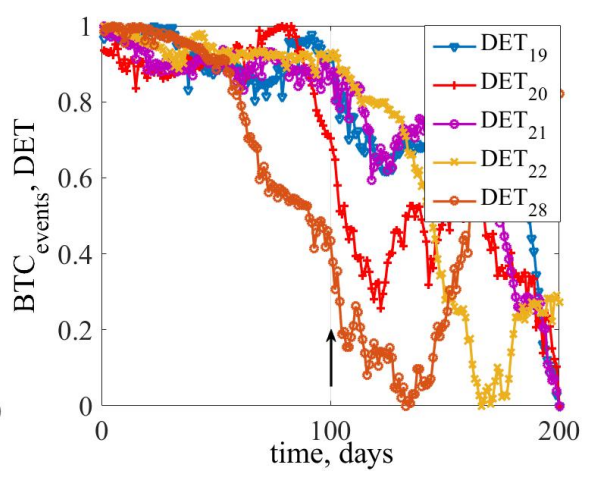

(h)

Figure 20: Dynamics of RR and DET for crashes (a, b, e, f) and critical events (c, d, g, h)

Another measure $\left(L_{\max }\right)$ considers the length of the longest diagonal line found in the RP. In other words, it means the maximum time that two segments of the trajec-

tory are close to each other, and the following equation can 


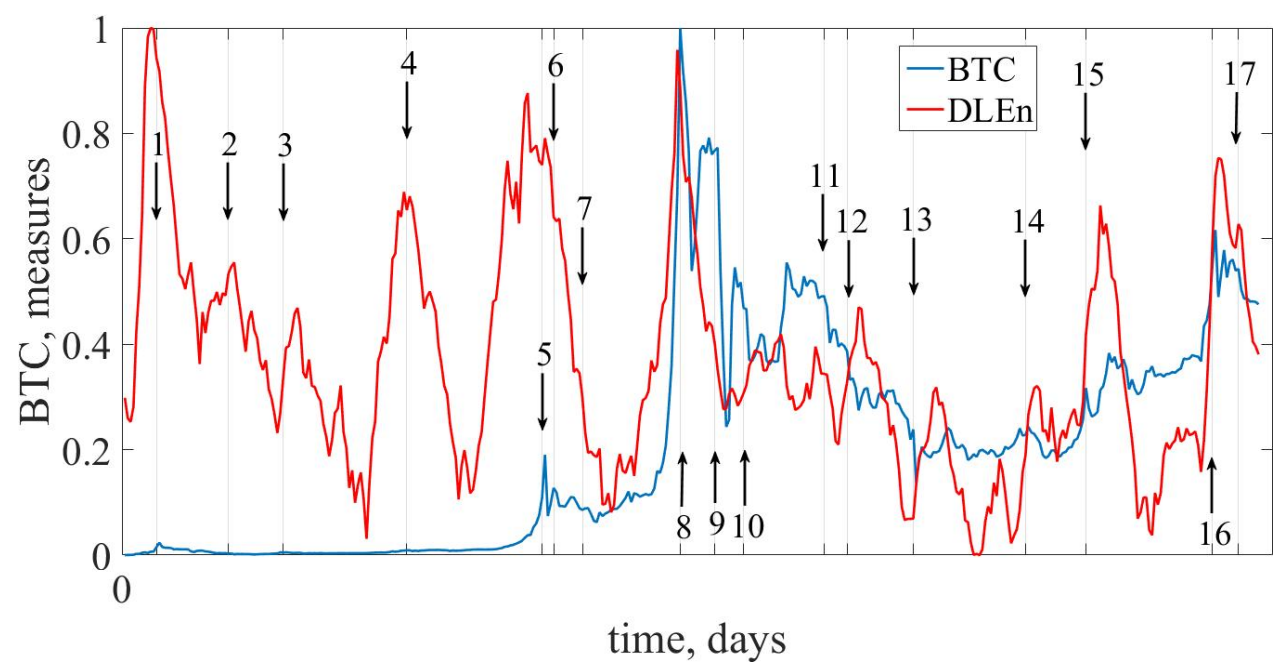

(a)

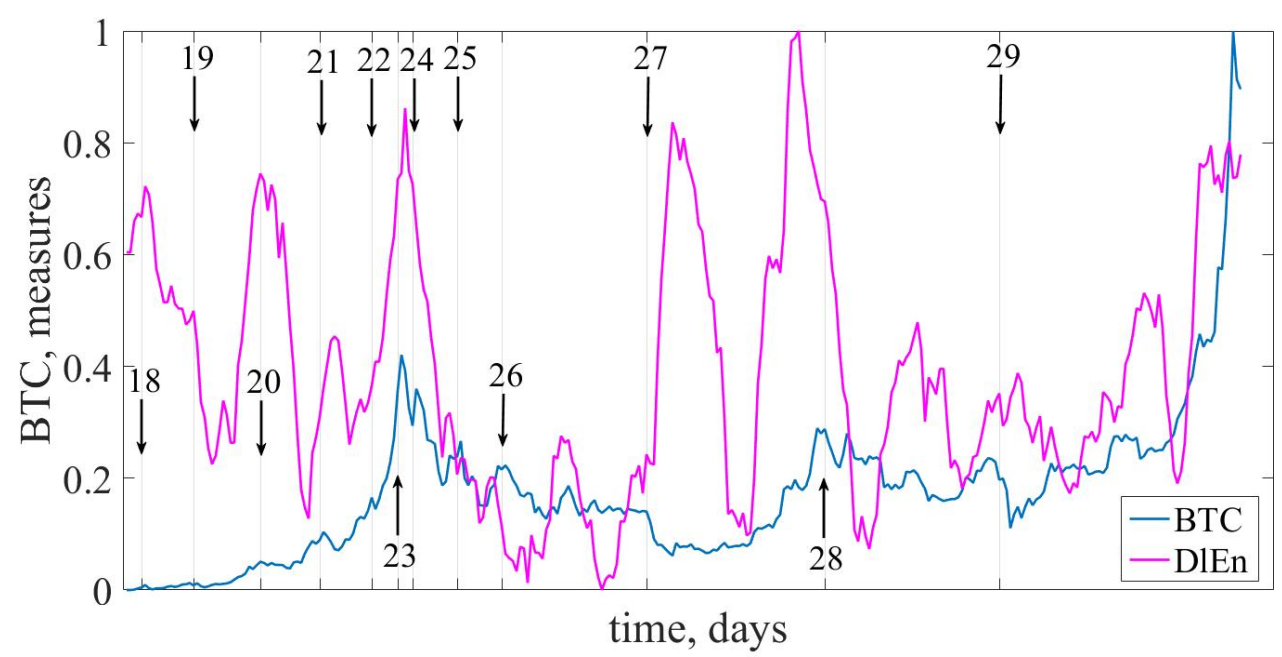

(b)

Figure 21: DLEn dynamics along with first (a) and second (b) periods of the entire time series of Bitcoin

be defined as

$$
L_{\max }=\max \left(\left\{l_{i} \mid i=1, \ldots, N_{l}\right\}\right),
$$

where $N_{l}=\sum_{l \geqslant l_{\min }} P(l)$ is the total number of diagonal lines.

Respectively, the inverse of $L_{\max }$ characterizes the exponential divergence of the phase space trajectory [301, 302]. Faster the trajectory segments diverge, shorter are the diagonal lines and higher is the measure of divergence $(D I V)$. It is given by the following equation:

$$
D I V=1 / L_{\max }
$$

Therefore, the measure of $D I V$, according to Eckmann [231], can be used to estimate the largest positive Lyapunov exponent. The comparative dynamics of the measure of divergence and BTC time series are presented in figure 24.

A comparative analysis of the measures under consideration revealed an obvious advantage of the recursive measure. In addition to the smoothness of the measure itself, it can be calculated for windows of small sizes, which leads to inaccurate or incorrect results for other methods.

\section{Irreversibility}

Complex systems are open systems that exchange energy, matter, and information with the environment. Investigating complex systems in the natural sciences, Prigogine made a fundamental generalization, indicating the need for consideration of the phenomena of irreversibility and non-equilibrium as principles of selection of space-time structures that are implemented in practice [303]. Later it became clear that this generalization extends to complex systems of another nature: social, economic, biomedical, etc. [304]. Prigogine believed that the most important changes in the modern scientific revolution are related to the removal of previous restrictions in the scientific understanding of time. The nonlinear world is characterized by features of temporality, i.e., irreversibility and transience 


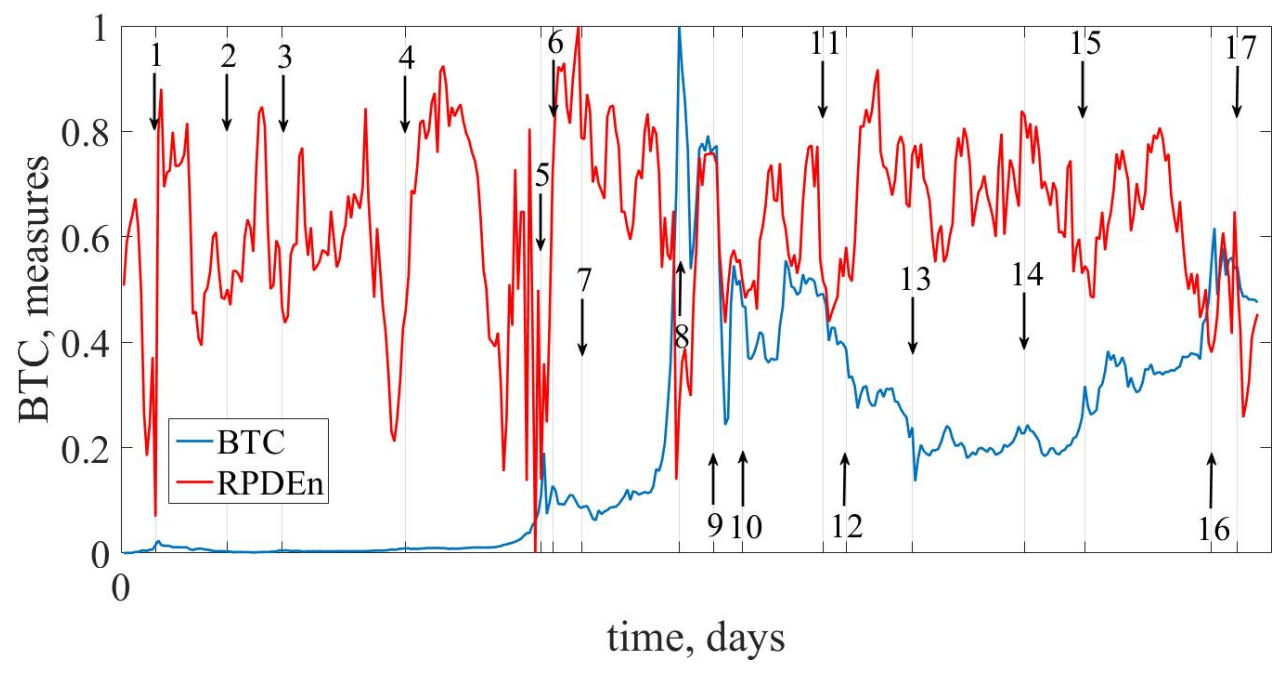

(a)

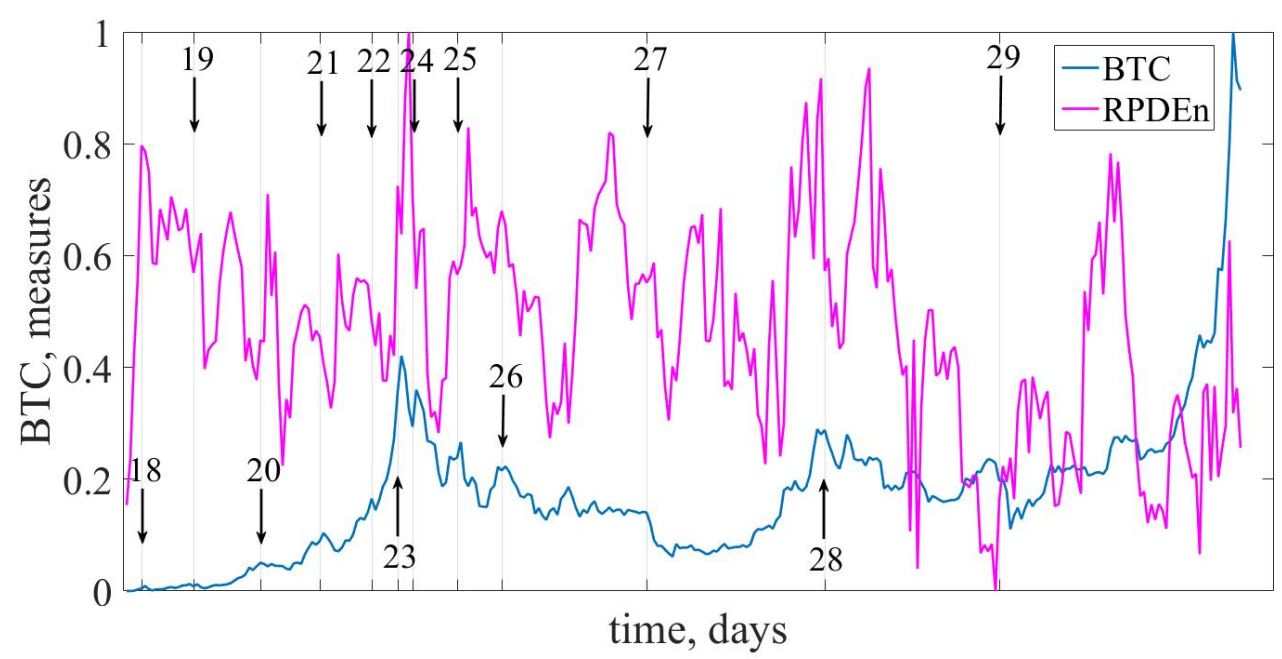

(b)

Figure 22: RPDEn dynamics along with first (a) and second (b) periods of the entire time series of Bitcoin

of processes and phenomena. Self-organization is considered as a spontaneous process of formation of integrating complex systems. It is due to the ambiguity of choice at bifurcation points that time in theories of self-organization becomes truly irreversible. In contrast to linear dynamic theories - classical, relativistic, quantum (where time is reversed), in the thermodynamics of dissipative structures created by Prigogine, time ceases to be a simple parameter and becomes a concept that expresses the pace and direction of events.

Thus, the irreversibility of time is a fundamental property of non-equilibrium dissipative systems, and its loss may indicate the development of destructive processes [107, 304].

A stationary process $\mathrm{X}$ is called statistically inverse in time, if for any $N$, the series $\{x(i) \mid i=1, \ldots, N\}$ and $\{x(i) \mid i=N, \ldots, 1\}$ will have the same compatible probability distributions [305]. The irreversibility of time series indicates the presence of nonlinearities in the dynamics of a system far from equilibrium, including non-Gaussian random processes and dissipative chaos. Since the definition of the irreversibility of the time series is formal, there is no a priori optimal algorithm for its quantification. Several methods for measuring the irreversibility of time have been proposed [107, 304, 306-312].

In the first group of methods, the symbolization of time series is performed, and then the analysis is performed by statistical comparison of the appearance of a string of symbols in the forward and reverse directions [307].

Sometimes additional compression algorithms are used [306]. An important step for this group is the symbolization - the conversion of the time series into a character series requires additional special information (e.g., division of the range or size of the alphabet) and, therefore, contains the problem of the algorithm's dependence on these additional parameters. The second problem arises when considering the large-scale invariance of complex signals. Since the procedures of typical symbolizations are local, taking into account different scales can cause some difficulties [107]. 


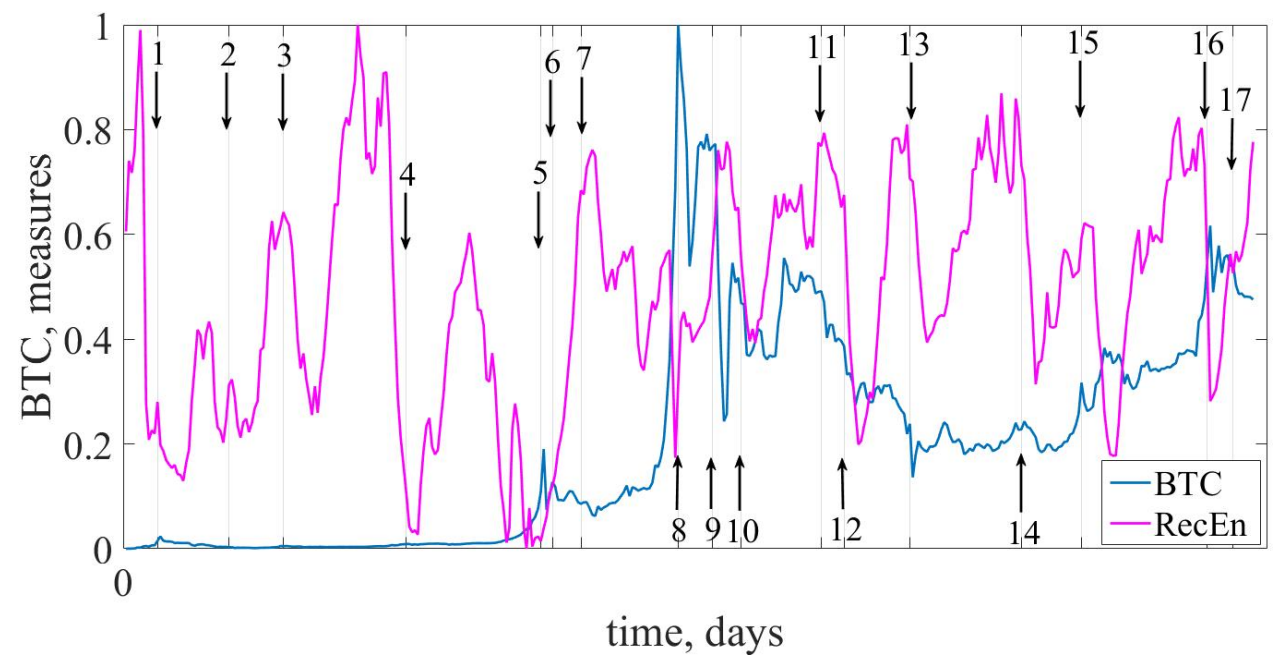

(a)

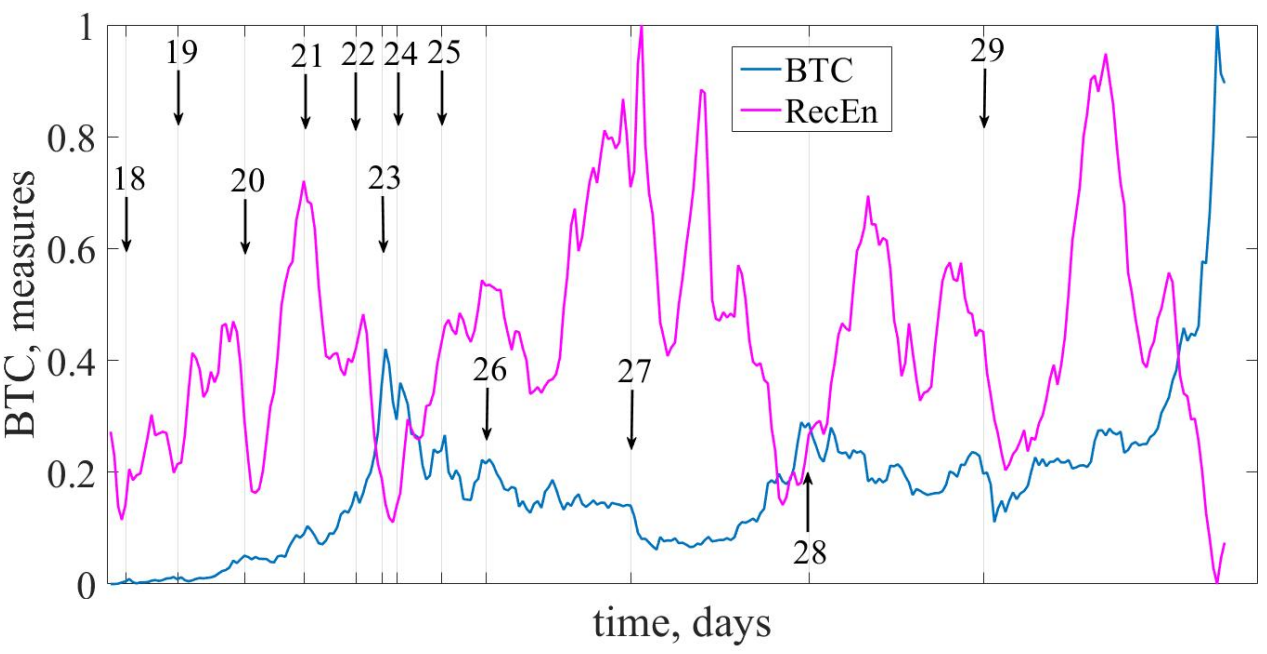

(b)

Figure 23: RecEn dynamics along with first (a) and second (b) periods of the entire time series of Bitcoin

Another group of methods in formalizing the index of irreversibility does not use the symbolization procedure but is based on the use of real values of the time series or returns.

One of such approaches is based on the asymmetry of the distribution of points in the Poincare map, built on the basis of the values of the analyzed time series [309, 312].

Recently, a fundamentally new approach to measuring the irreversibility of time series has been proposed, which uses the methods of complex network theory [308, 311] and which combines two tools: the algorithm for visibility of time series recovery into a complex network and the Kullbak-Leibler divergence algorithm [311]. The first forms a directional network according to the geometric criterion. The degree of irreversibility of the series is then estimated by the Kullbak-Leibler divergence (i.e., the resolution) between the distribution of the input and output stages of the associated count. This method is computationally efficient, does not require any special symboliza- tion of the process, and, according to the authors, naturally takes into account multiscale.

In this study, we consider the irreversibility of time as a measure of the complexity of the system.

Let us consider non-reversible measures of complexity based on the construction and analysis of ordinal permutation patterns.

\subsection{Time series irreversibility measure based on permutation patterns}

The concept of permutation patterns (PP) was introduced by Bandt and Pompe [127]. PP is based on the idea of finding the order patterns that result in sorted (ascending) sub-sequences, and of then studying the probability distribution of these patterns. Authors of the work [313] introduce a new method, based on permutation entropy $[127,314,315]$, to evaluate irreversibility of time series at various temporal scales. The proposed one presents various advantages: (1) it has no free parameters other than the 


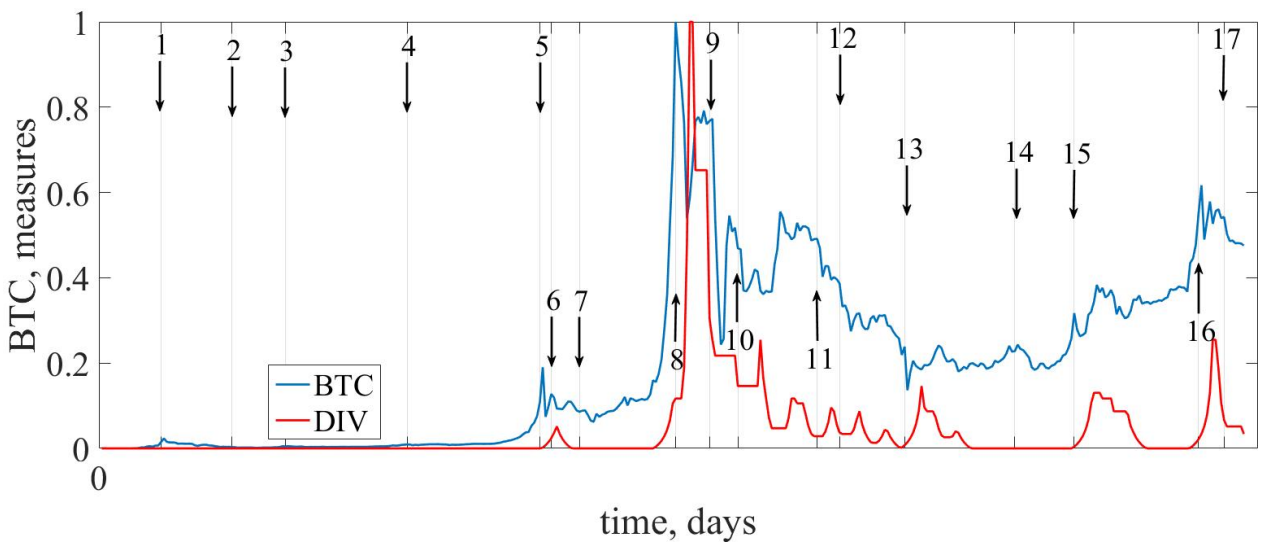

(a)

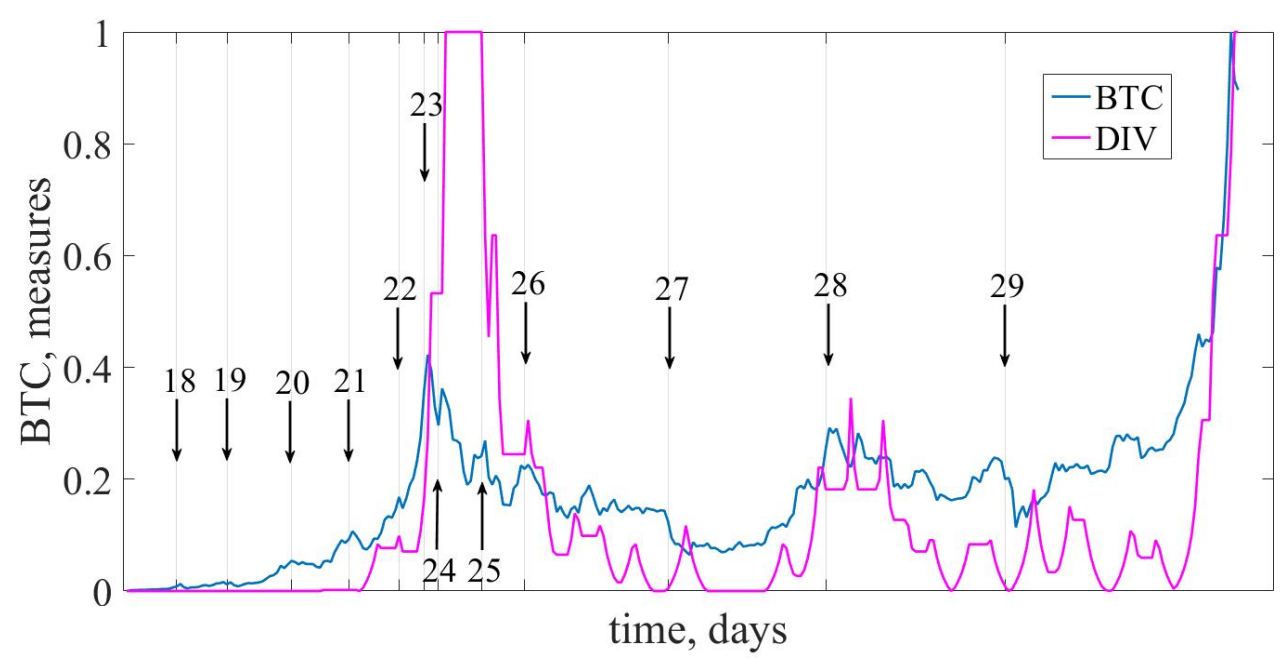

(b)

Figure 24: The measure of divergence along with first (a) and second (b) periods for BTC time series

embedding dimension of the permutation entropy; (2) similar to visibility graph methods $[311,316]$, it is temporally local, and therefore allows assessing fluctuations; (3) assessing significance is straightforward, and does not rely on scaling arguments as in visibility graph methods; and (4) it has a convergence speed advantage over visibility graph methods graph methods. They also demonstrated how the proposed approach can help elucidate the complex irreversibility dynamics of financial time series, representing 30 major European stocks and 12 world indices [313] and gait analysis on late stages of neurodegenerative dementias [317]. This technique was also used to analyze the irreversibility of time series in ecology (the time series of lynx abundance), epidemiology (dengue prevalence), economy (the S\&P price-index series), neuroscience (electroencephalographic data from an epileptic patient) [318, 319], and heart rate [320].

The idea of irreversibility analysis implies that regarding each permutation pattern that can be obtained following procedure from section 3.2.4, there will be reversed one under the operation of time reversal. Example, if for an embedded vector $\vec{X}$, a pattern $\pi_{i}$ is found, reversing time series will necessary imply $\pi_{i}^{r}$. As an example, let us consider a fragment of BTC time series for the period 21.01.2021-31.01.2021:

$\mathrm{X}^{d}=[30825.70,33005.76,32067.64,32289.38,32366.39]$ and

$\mathrm{X}^{r}=[32366.39,32289.38,32067.64,33005.76,30825.70]$.

According to mentioned steps, we will construct embedded matrix of overlapping column vectors with $d_{E}=3$ and $\tau=1$. Our sampled data is partitioned as follows:

$$
\mathrm{X}_{t}^{d}\left(d_{E}, \tau\right)=\left[\begin{array}{lll}
30825.70 & 33005.76 & 32067.64 \\
33005.76 & 32067.64 & 32289.38 \\
32067.64 & 32289.38 & 32366.39
\end{array}\right]
$$

and for $\mathrm{X}_{t}^{r}\left(d_{E}, \tau\right)$ :

$$
\left[\begin{array}{lll}
32366.39 & 32289.38 & 32067.64 \\
32289.38 & 32067.64 & 33005.76 \\
32067.64 & 33005.76 & 30825.70
\end{array}\right] .
$$

After it, our time-delayed vectors are mapped to permutations or ordinal patterns of the same size. Our example consists $3 !=6$ different ordinal patterns in total. They 
can be paired together, such that each pattern composing a pair is the time reversal of the other. For instance:

$$
\begin{aligned}
& \{0,1,2\} \leftrightarrow\{2,1,0\} \\
& \{1,0,2\} \leftrightarrow\{2,0,1\} \\
& \{1,2,0\} \leftrightarrow\{0,2,1\}
\end{aligned}
$$

with $\leftrightarrow$ representing a time reversal transformation.

As an example, the corresponding permutation of the first column from (39) would be $\phi(30825.70,33005.76,32067.64])=021$ since we arrange values in ascending order and replace them by their ordinal ranking from original placement. Therefore, after mapping from the time-series data into a series of permutations $\left(\phi: \mathbb{R}^{d_{E}} \rightarrow S_{d_{E}}\right.$ ), we obtain the ordinal matrices first of all for initial time series:

$$
\left[\begin{array}{lll}
0 & 1 & 0 \\
2 & 2 & 1 \\
1 & 0 & 2
\end{array}\right]
$$

and its reversed version:

$$
\left[\begin{array}{lll}
2 & 1 & 1 \\
1 & 0 & 2 \\
0 & 2 & 0
\end{array}\right]
$$

Finally, the probability of each pattern in initial and reversed time series is calculated as

$$
p(\pi)=\frac{\#\left\{t \leq N-\left(d_{E}-1\right) \tau, \phi\left(\mathrm{X}_{t}^{d_{E}, \tau}\right)=\pi\right\}}{N-\left(d_{E}-1\right) \tau},
$$

forming the probability distributions $P^{d}=\left[p_{0,1,2}, p_{2,1,0}, p_{1,0,2}, p_{2,0,1}, p_{1,2,0}, p_{0,2,1}\right]$ and $P^{r}=\left[p_{2,1,0}, p_{0,1,2}, p_{2,0,1}, p_{1,0,2}, p_{0,2,1}, p_{1,2,0}\right]$ Using the Kullback-Leibler divergence, we can define the degree of irreversibility in a time series:

$$
D_{K L}=\sum_{i=1}^{d_{E} !} P^{d}(i) \log \frac{P^{d}(i)}{P^{r}(i)} .
$$

If $P^{d} \approx P^{r}$, the time series is presented to be reversible, thus yielding a $D_{K L} \approx 0$ and vise versa. Estimating varying bitcoin's irreversibility according to (44) with time window of 100 days and time step of 1 day, we obtain following results.

The figures show that time series are significantly irreversible. When moving the original rows of their irreversible disappears. Draws attention and noticeable unevenness introduced measures, which correlate with the fluctuations of the input time series. Identifying significant changes in the time series and comparing them with the corresponding changes of non-reversible measures of complexity, it is possible to construct the corresponding indicators.

\section{Single and multiplex networks}

The new interdisciplinary study of complex systems, known as the complex networks theory, laid the foundation for a new network paradigm of synergetics [321]. In the framework of the complexity paradigm, it became apparent that we should move from well-studied systems and processes, taking into account the minimal number of new entities that are characteristic of the social sciences or the humanities. Apparently, one of these entities is the bonds, that is, what characterizes the interaction of the elements that are part of the system, that makes parts of the whole. The set of these links is called the network. Investigating networks, we take into account their topology, statistical properties, the distribution of weights of individual nodes and edges, the effects of information dissemination, robustness, etc. [1, 4, 322, 323]. Complex networks include electrical, transport, information, social, economic, biological, neural, and other networks [3, 324, 325]. The network paradigm has become dominant in the study of complex systems since it allows you to enter new quantitative measures of complexity not existing for the time series [326]. Moreover, the network paradigm provides adequate support for the core concepts of Industry 4.0 [321].

Previously, we introduced various quantitative measures of complexity for individual time series [57, 327329]. However, except a graph for an individual time series, it is necessary to take into account the interconnection interaction, which can be realized within the framework of different models [330]. We will consider it by simulating so-called multiplex networks, the features of which are reduced to a fixed number of nodes in each layer, but they are linked by different bonds [330].

Recently, the first papers using the spectral and topological characteristics of dynamic systems presented as networks have appeared. Thus, in [331], it has been investigated universal and non-universal allometric scaling behaviors in the visibility graphs of 30 world stock market indices. It has been established that the nature of such behavior is due to the returns distribution that is characterized by fat-tails, the nonlinear long-term correlation, and a coupling effect between the set of influential factors.

Birch [332] compared the mean degree value and clustering coefficient for a group of companies included in the DAX 30 index basket. He observed the companies from the DAX 30 index for two time periods: the first from the beginning of 2008 through the end of 2009 and the second from the beginning of 2010 up to the end of 2011 as these include the dates - a period of crisis (7th October 2008 - 31st December 2008) and a period of recovery (7th May 2010 - 3rd August 2010). Contrary to expectations, the results differed little from the relatively low accuracy of the horizontal visibility graph procedure compared to visibility graph.

Wand and Wei [333] collected from the Chinese stock market the data of 2571 stock companies in 2012 and the data of 2578 stock companies in 2013. Every year, data of these stock companies are randomly arranged. These data are then converted into some complex networks based on the visibility graph method. For these complex networks, degree distribution and clustering coefficient are considered. These results show that complex networks have power-law distribution and small-world properties.

Yan and Serooskerken [334] construct an indicator to measure the magnitude of the super-exponential growth of 


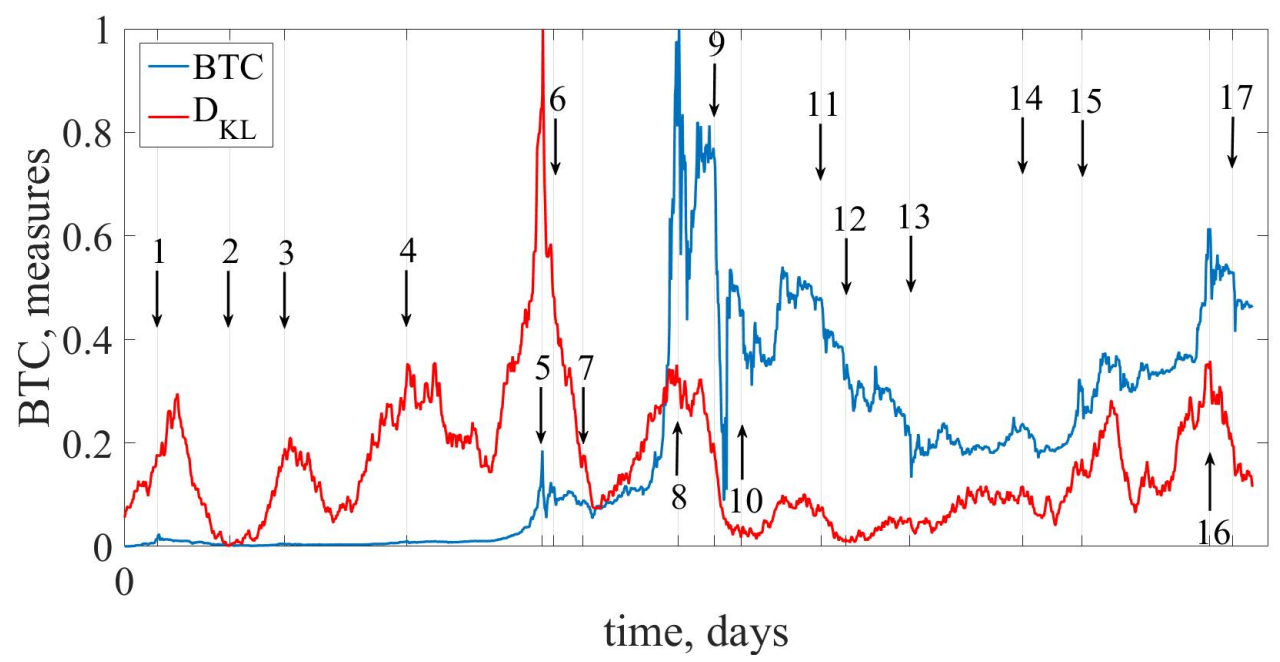

(a)

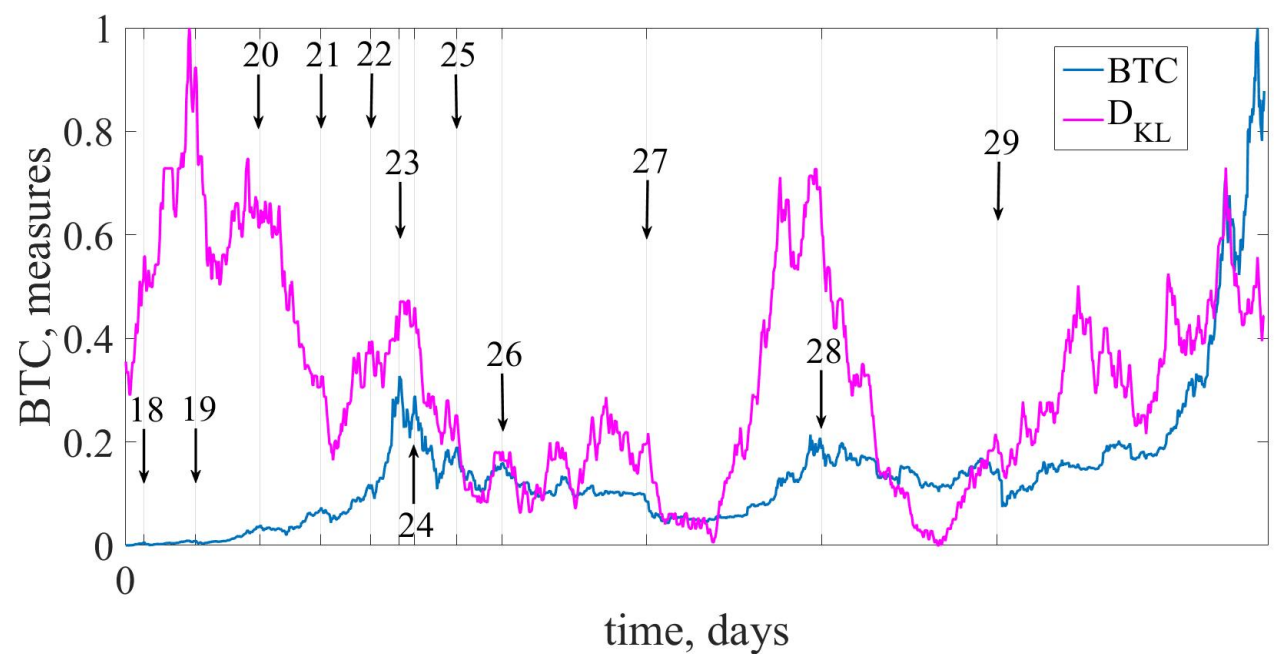

(b)

Figure 25: Dynamics of permutation-based time irreversibility measure for the first (a) and second (b) periods

stock prices, by measuring the degree of the price network, generated from the price time series. Twelve major international stock indices have been investigated. The work results show that this new indicator has strong predictive power for financial extremes, both peaks, and troughs. By varying the model parameters, the authors show the predictive power is very robust. The new indicator has a better performance than the indicator based on a well-known model of log-periodic oscillations of Sornette [335].

Authors of another paper [336] analyze highfrequency data from the S\&P 500 via the horizontal visibility graph method and find that all major crises that have taken place worldwide for the last twenty years, affected significantly the behavior of the price-index. Nevertheless, they observe that each of those crises impacted the index in a different way and magnitude. These results suggest that the predictability of the price-index series increases during periods of crisis.

In the paper [337] Serafino et al. studied the visibility graphs built from the time series of several stock market indices. They proposed a validation procedure for each link of these graphs against a null hypothesis derived from ARCH-type modeling of such series. Building on this framework made it possible to devise a market indicator that turned out to be highly correlated and even predictive of financial instability periods.

Francés and Carles [25] examined the characteristics of the daily price series of 16 different cryptocurrencies between July 2017 and February 2018. Using Minimum Spanning Tree (MST) and hierarchical analysis by dendrograms that were obtained from Pearson correlation between daily returns, they visualized and identified a high correlation between price movements of all the currencies. From the obtained results, it is seen that the most interconnected with all cryptocurrencies is Ethereum, while Bitcoin standed for one of its branches. They concluded that Ethereum might be a benchmark currency in the cryptocurrency market, rather than Bitcoin.

Coquide et al. [338] constructed the Google matrices of bitcoin transaction for all year quarters from the 
very start of it till April 10, 2013. From PageRank and CheiRank probabilities that serve as analogues to trade import and export, they determined the dimensionless trade balance of each user and analyzed the direct and hidden (indirect) links between top PageRank users of BCN using the recently developed reduced Google matrix algorithm. They modeled the contagion propagation of the transactions assuming that a user goes bankrupt if its dimensional balance exceeds a certain bankruptcy threshold $k$. Their results present that the phase transition neighboring with the critical threshold $k=k_{c} \approx 0.1$ below which almost all users remain safe. For $k>0.55$ almost all users remain safe, and for $0.1<k>0.55$ more than a half of users go bankrupt. Moreover, their result present that even being not very close to the critical threshold $k_{c} \approx 0.1$, almost all top PageRank and CheiRank users rapidly become bankrupts that give the evidence about their strong interconnectivity. With the reduced Google matrix algorithm, they presented the most preferable interlinks of the most valuable users.

Multiplex networks are actively used to simulate complex networks of different nature: from financial (stock market [336, 337, 339, 340], banks [341], guarantee market [342]) to social [343]. Particular attention should be paid to the work [339], in which the above multiplex measures are analyzed for the subject of correlations with known stock market crises.

\subsection{Methods of converting time series into graphs}

In recent years, interesting algorithms for the transformation of time series into a network have been developed, which allows extending the range of known characteristics of time series even to network ones. Recently, several approaches have been proposed to transform time sequences into complex network-like mappings. These methods can be conventionally divided into three classes [344].

The first is based on the study of the convexity of successive values of the time series and is called visibility graph (VG) $[344,345]$. The second analyzes the mutual approximation of different segments of the time sequence and uses the technique of recurrent analysis [344]. The recurrent diagram reflects the existing repetition of phase trajectories in the form of a binary matrix whose elements are units or zeros, depending on whether they are close (recurrent) with given accuracy or not, the selected points of the phase space of the dynamic system. The recurrence diagram is easily transformed into an adjacency matrix, on which the spectral and topological characteristics of the graph are calculated. Finally, if the basis of forming the links of the elements of the graph is to put correlation relations between them, we obtain a correlation graph [327, 344]. To construct and analyze the properties of a correlation graph, we must form an adjacency matrix from the correlation matrix. To do this, you need to enter a value that for the correlation field will serve as the distance between the correlated agents. Such a distance may be dependent on the ratio of the correlation $C_{i j}$ value $d_{i j}=\sqrt{2\left(1-C_{i j}\right)}$. So, if the correlation coefficient between the two assets is significant, the distance between them is small, and, starting from a certain critical value $x_{c r i t}$, assets can be considered bound on the graph. For an adjacency matrix, this means that they are adjacent to the graph. Otherwise, the assets are not contiguous. In this case, the binding condition of the graph is a prerequisite.

The main purpose of such methods is to accurately reproduce the information stored in the time series in an alternative mathematical structure, so that powerful graph theory tools could eventually be used to characterize the time series from a different point of view in order to overcome the gap between nonlinear analysis of time series, dynamic systems and the graphs theory.

The usage of the complexity of recurrent networks to prevent critical and crisis phenomena in stock markets has been considered by us in a recent paper [346]. Therefore, we will focus on algorithms of the VG and multiplex VG (MVG).

The recurrence diagrams for the visualization of phase space recurrences are based on Henri Poincare's idea of the phase space recurrence of dynamical systems. According to Takens' theorem [136], an equivalent phase trajectory that preserves the structure of the original phase trajectory can be recovered from a single observation or time series by the time delay method: the recurrence diagram is easily transformed into an adjacency matrix, by which the spectral and topological characteristics of the graph are calculated [327].

The algorithm of the VG is realized as follows [345].

Take a time series $\left\{x\left(t_{i}\right) \mid i=1, \ldots, N\right\}$ of length $N$. Each point in the time series data can be considered as a vertex in an associative network, and the edge connects two vertices if two corresponding data points can "see" each other from the corresponding point of the time series (see Figure 26). Formally, two values $x\left(t_{a}\right)$ (at a point in time $t_{a}$ ) and $x\left(t_{b}\right)$ (at a point in time $t_{b}$ ) are connected, if, for any other value $\left(x\left(t_{c}\right), t_{c}\right)$, which is placed between them (i.e., $t_{a}<t_{c}<t_{b}$ ), the condition is satisfied:

$$
x\left(t_{c}\right)<x\left(t_{a}\right)+\left(x\left(t_{b}\right)-x\left(t_{a}\right)\right) \frac{t_{c}-t_{a}}{t_{b}-t_{a}} .
$$

Note that the visibility graph is always connected by definition and also is invariant under affine transformations, due to the mapping method.

An alternative (and much simpler) algorithm is the horizontal visibility graph (HVG) [344], in which a connection can be established between two data points $a$ and $b$ if one can draw a horizontal line in the time series joining them that does not intersect any intermediate data by the following geometrical criterion: $x\left(t_{a}\right), x\left(t_{b}\right)>x\left(t_{c}\right)$ for all $c$ such that $t_{a}<t_{c}<t_{b}$ (see figure 26).

In multiplex networks, there are two tasks [346]: (1) turn separate time series into the network for each layer; (2) connect the intra-loop networks to each other. The first problem is solved within the framework of the standard algorithms described above. For multiplex networks, the algorithm of the MVG for the three layers is presented in figure 27.

The cross-recurrent multiplex network (MCRP) is formed from recursive diagrams of individual layers. 


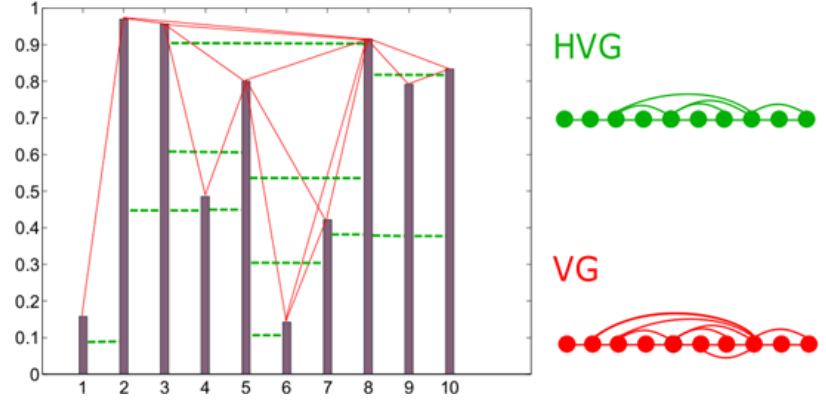

Figure 26: Illustration of constructing the visibility graph (red lines) and the horizontal visibility graph (green lines) [347]

\subsection{Spectral and topological graph properties}

Spectral theory of graphs is based on algebraic invariants of a graph - its spectra [27]. The spectrum of graph $G$ is the set of eigenvalues $S_{p}(G)$ of a matrix corresponding to a given graph. For adjacency matrix $A$ of a graph, there exists a characteristic polynomial $|\lambda I-A|$, which is called the characteristic polynomial of a graph $P_{G}(\lambda)$. The eigenvalues of the matrix $A$ (the zeros of the polynomial $|\lambda I-A|)$ and the spectrum of the matrix $A$ (the set of eigenvalues) are called respectively their eigenvalues.

Another common type of graph spectrum is the spectrum of the Laplace matrix $L$. The Laplace matrix is used to calculate the tree graphs, as well as to obtain some important spectral characteristics of the graph. It can be defined as $L=D-A$ where $D-$ diagonal matrix of order $n$ :

$$
d_{i j}= \begin{cases}d_{i}, & i=j, \\ 0, & i \neq j,\end{cases}
$$

with $d_{i}$ - the degree of corresponding vertex in the graph.

The spectrum $S_{p_{L}}(G)$ of the matrix $L$ is the root of the characteristic equation:

$$
|\lambda I-L|=|\lambda I-D+A|=0
$$

Comparing the spectra of $S_{p}$ with $S_{p_{L}}$, it is easy to establish that

$$
\begin{aligned}
S_{p}(G) & =\left[\lambda_{1}, \lambda_{2}, \ldots, \lambda_{n}\right], \\
S_{p_{L}}(G) & =\left[r-\lambda_{n}, \lambda_{n-1}, \ldots, r-\lambda_{1}\right],
\end{aligned}
$$

where $\lambda_{1}=r$.

The number zero is the eigenvalue of the matrix $L$, which corresponds to an eigenvector whose coordinates are equal to unity. The multiplicity of the null eigenvalue is equal to the number of connected components of the graph. The rest of eigenvalues $L$ are positive. The least of the positive eigenvalues $\lambda_{2}$ is called the index of algebraic connectivity of the graph. This value represents the "force" of the connectivity of the graph component and is used in the analysis of reliability and synchronization of the graph.

Important derivative characteristics are spectral gap, graph energy, spectral moments, and spectral radius. The spectral gap is the difference between the largest and the next eigenvalues of the adjacency matrix and characterizes the rate of return of the system to the equilibrium state. The graph energy is the sum of the modules of the eigenvalues of the graph adjacency matrix:

$$
E(G)=\sum_{i=1}^{n}\left|\lambda_{i}\right| .
$$

The spectral radius is the largest modulus of the eigenvalue of the adjacency matrix. Denote by $N_{c}$ the value, which corresponds to an average eigenvalue of the graph adjacency matrix:

$$
N_{c}=\ln \left(\frac{1}{n} \sum_{i=1}^{n} \exp \left(\lambda_{i}\right)\right)
$$

and is called natural connectivity.

The $k^{\text {th }}$ spectral moment of the adjacency matrix is determined by the expression

$$
m_{k}(A)=\frac{1}{n} \sum_{i=1}^{n} \lambda_{i}^{k},
$$

with $\lambda_{i}$ that represents eigenvalues of the adjacency matrix, and $n$ is the vertex of $G$.

Among the topological measures, one of the most important is the node degree $k$ - the number of links attached to this node. For non-directed networks, the node's degree $k_{i}$ is determined by the sum $k_{i}=\sum_{j} a_{i j}$, where the elements $a_{i j}$ of the adjacency matrix.

For characterizing the linear size of the network, there are useful concepts of average $\langle l\rangle$ and maximum $l_{\text {max }}$ shortest paths. For a connected network of $n$ nodes, the average path length (ApLen) is equal to

$$
\langle l\rangle=\frac{2}{n(n-1)} \sum_{i>j} l_{i j},
$$

where $l_{i j}$ - the length of the shortest path between the nodes. The diameter of the connected graph is the maximum possible distance between its two vertices, while the minimum possible is the radius of the graph.

If the average length of the shortest path gives an idea of the whole network and is a global characteristic, the next parameter - the clustering coefficient - is a local value and characterizes a separate node. For a given node $i$, the clustering coefficient $C_{i}$ is defined as the ratio of the existing number of links between its closest neighbors to the maximum possible number of such relationships:

$$
C_{i}=\frac{2 E_{i}}{k_{i}\left(k_{i}-1\right)}
$$

In equation (48), $k_{i}\left(k_{i}-1\right) / 2$ is the maximum number of links between the closest neighbors. The clustering coefficient of the entire network is defined as the average value of $C_{i}$ for all its nodes. The clustering coefficient shows how many of the nearest neighbors of the given node are also the closest neighbors to each other. It characterizes the tendency to form groups of interconnected nodes clusters. For the real-life networks, the high values of the clustering coefficient are high. 


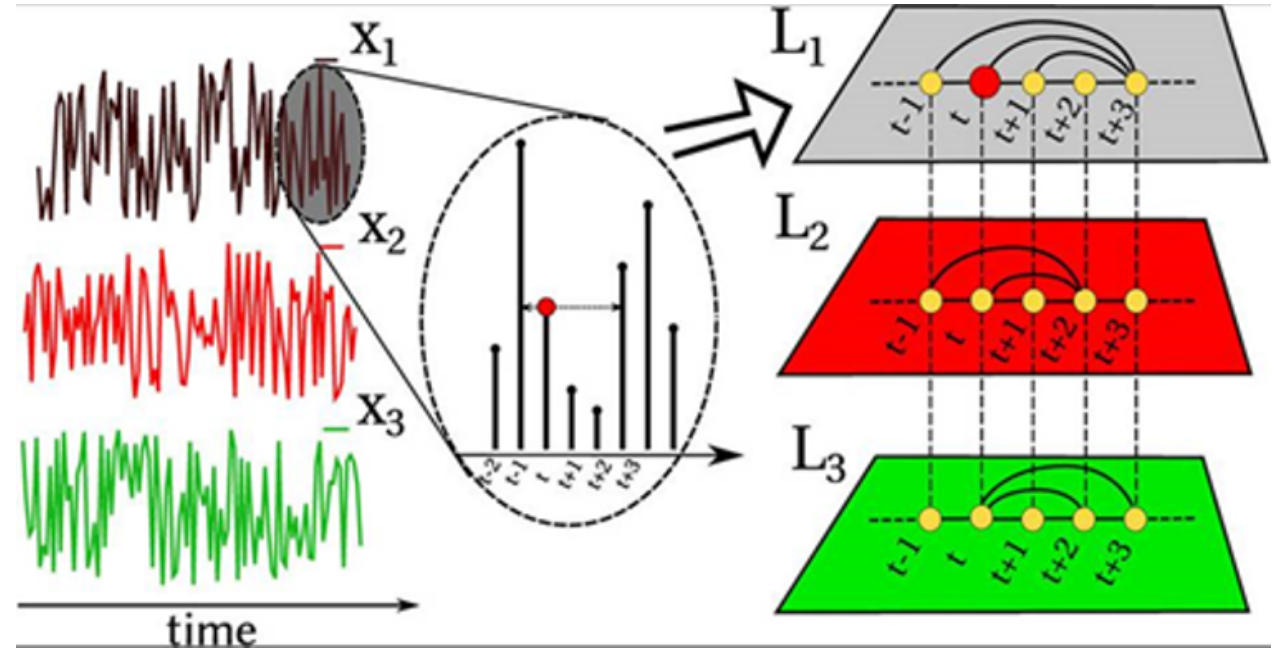

Figure 27: Scheme for forming bonds between three layers of the multiplex network [346]

Another feature of the node is betweenness. It reflects the role of the node in establishing network connections and shows how many shortest paths pass through this node. Node betweenness $c(v))$ is defined as

$$
c(v)=\sum_{i \neq j} \frac{\sigma(i, j \mid v)}{\sigma(i, j)},
$$

where $\sigma(i, j)$ - the total number of shortest paths between nodes $i$ and $j ; \sigma(i, j \mid v)$ - the number of those shortest paths between $i, j$ passing through $v$. The value of (49) is also called the load or betweenneess centrality.

One of the main characteristics of the network is the degree distribution $P(d)$, which is defined as the probability that the node $i$ has a degree $d_{i}=d$. Most natural and actual artificial networks follow a power law distribution

$$
P(d) \sim 1 / d^{\gamma}, d \neq 0, \gamma>0 \text {. }
$$

Also, important topological characteristic is the vertex eccentricity - the largest distance between $i$ and any other vertex, that is, how far the vertex is far from the other vertices of the graph. The centrality of the vertex measures its relative importance in the graph. At the same time, the farness of a node is defined as the sum of its distances to all other nodes, and its closeness is defined as the backward distance. Thus, the centrality of the node is lower than its total distance to all other nodes.

Another important measure is the link density in the graph, which is defined as the number of links $n_{e}$, divided by the expression $n_{n}\left(n_{n}-1\right) / 2$, where $n_{n}$ is the number of nodes of the graph.

From spectral measures, we consider the maximal node degree $\left(d_{\max }\right.$ - figure $28 \mathrm{a}$ and figure $28 \mathrm{~b}$ ). From the topological measures, the average path length (APLen figure $28 \mathrm{c}$ and 28d) is found, which is in accordance with equation (47).

Figure 28 demonstrates the asymmetric response of the spectral and topological measures of network complexity. For the complete series, the calculation parameters are as follows: window length of 100 days, step is of 1 day.
Figures above show that all of the above spectral measures have maximum values in pre-crisis periods. The complex system has the greatest complexity. With the approach of the crisis, the complexity of the system decreases, recovering after the crisis. Some of the topological, in particular, APLen, shows an opposite relationship. Indeed, in more complex systems you can always find shorter paths that connect any nodes. During the crisis (reducing complexity, increasing the chaotic component), the length of the corresponding path increases.

\subsection{Multiplex complexity measures}

A multilayer/multiplex network [83, 348] is a pair $M=(G, C)$ where $\left\{G_{\alpha} \mid \alpha \in 1, \ldots, M\right\}$ is a family of graphs (whether directed or not, weighed or not) $G_{\alpha}=\left(X_{\alpha}, E_{\alpha}\right)$ that called layers and

$$
C=\left\{E_{\alpha \beta} \subseteq X_{\alpha} \times X_{\beta} \mid \alpha, \beta \in 1, \ldots, M, \alpha \neq \beta\right\}
$$

is a set of links between nodes of layers $G_{\alpha}$ and $G_{\beta}$ where $\alpha \neq \beta$. The elements of each $E_{\alpha}$ are intralayer edges in $M$ in contrast to the elements of each $E_{\alpha \beta}$ that called interlayer edges.

A set of nodes in a layer $G_{\alpha}$ is denoted as $X_{\alpha}=\left\{x_{1}^{\alpha}, \ldots, x_{N_{\alpha}}^{\alpha}\right\}$, and an intralayer adjacency matrix as $A^{[\alpha]}=\left(a_{i j}^{\alpha}\right) \in \mathrm{Re}^{N_{\alpha} \times N_{\alpha}}$, where

$$
\alpha_{i j}^{\alpha}= \begin{cases}1, & \left(x_{i}^{\alpha}, x_{j}^{\alpha}\right) \in E_{\alpha} \\ 0, & \text { otherwise }\end{cases}
$$

for $1 \leq i \leq N_{\alpha}, 1 \leq j \leq N_{\beta}$ and $1 \leq \alpha \leq M$. For an interlayer adjacency matrix, we have $A^{[\alpha, \beta]}\left(a_{i j}^{\alpha \beta}\right) \in \operatorname{Re}^{N_{\alpha} \times N_{\beta}}$, where

$$
\alpha_{i j}^{\alpha \beta}= \begin{cases}1, & \left(x_{i}^{\alpha}, x_{j}^{\beta}\right) \in E_{\alpha \beta}, \\ 0, & \text { otherwise. }\end{cases}
$$

A multiplex network is a partial case of interlayer networks, and it contains a fixed number of nodes connected 


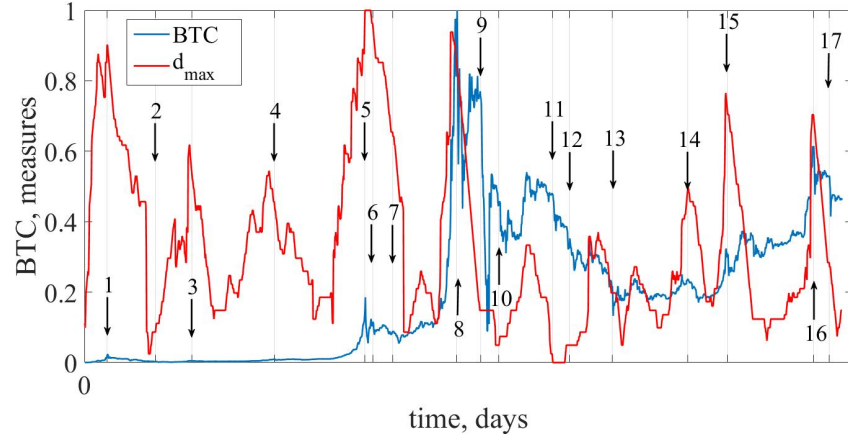

(a)

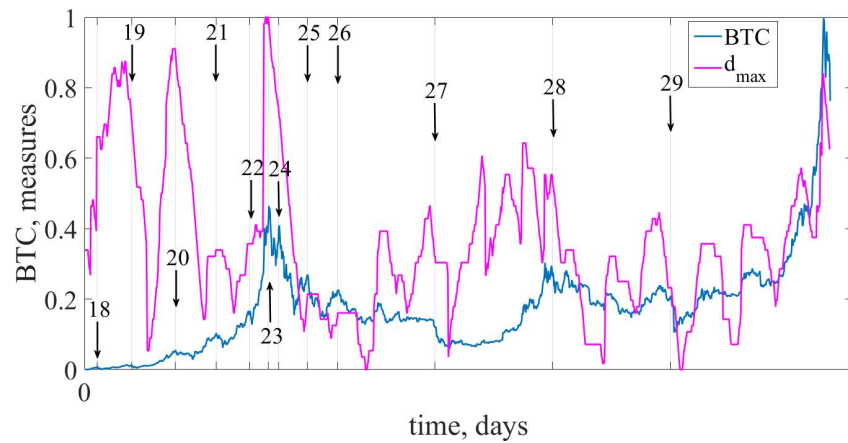

(b)

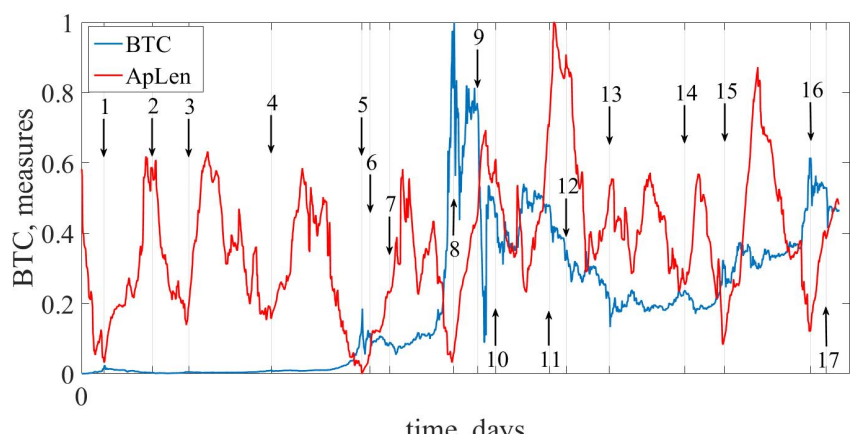

(c)

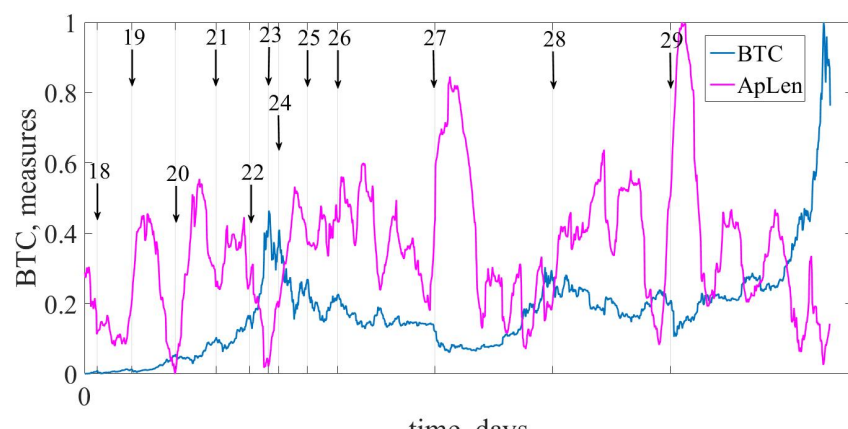

(d)

Figure 28: Maximal node degree $d_{\max }(\mathrm{a}, \mathrm{b})$ and average path length $\langle l\rangle(\mathrm{c}, \mathrm{d})$ for two periods of Bitcoin time series

by different types of links. Multiplex networks are characterized by correlations of different nature [330], which enable the introduction of additional multiplexes.
Let's evaluate the quantitative overlap between the various layers. The average edge overlap is defined as [340]

$$
\omega=\frac{\sum_{i} \sum_{j>i} \sum_{\alpha} a_{i j}^{[\alpha]}}{M \sum_{i} \sum_{j} 1-\delta_{0, \sum_{\alpha} \alpha_{i j}^{[\alpha]}}}
$$

and determines the number of layers in which this edge is presented. Its value lies on the interval $[1 / M, 1]$ and equals $1 / M$ if the connection $(i, j)$ exists only in one layer. In other words, if there is a layer $\alpha$ such that $a_{i j}^{[\beta]}=0 \forall \beta \neq \alpha$. If all layers are identical, then $\omega=1$. Consequently, this measure can serve as a measure of the coherence of the output time series: high values of $\omega$ indicate a noticeable correlation in the structure of the time series.

The total overlap $O^{\alpha \beta}$ between the two layers $\alpha$ and $\beta$ is defined as the total number of edges that are shared between the layers $\alpha$ and $\beta$ :

$$
O^{\alpha \beta}=\sum a_{i j}^{\alpha} a_{i j}^{\beta}
$$

where $\alpha \neq \beta$.

For a multiplex network, the node degree $k$ is already a vector

$$
k_{i}=\left(k_{i}^{[1]}, \ldots, k_{i}^{[M]}\right),
$$

with the degree $k_{i}^{[\alpha]}$ of the node $i$ in the layer $\alpha$, namely

$$
k_{i}^{[\alpha]}=\sum_{j} a_{i j}^{[\alpha]}
$$

while $a_{i j}^{[\alpha]}$ is the element of the adjacency matrix of the layer $\alpha$. Specificity of the node degree in vector form allows to describe additional quantities. One of them is the ovelapping degree of node $i$

$$
o_{i}=\sum_{\alpha=1}^{M} k_{i}^{[\alpha]} .
$$

The next measure quantitatively describes the interlayer degree correlations of the selected node in two different layers. If a chosen pair $(\alpha, \beta)$ from $M$ layers is characterized by the distributions $P\left(k^{[\alpha]}\right), P\left(k^{[\beta]}\right)$, the so-called interlayer mutual information is determined as:

$$
I_{\alpha, \beta}=\sum \sum P\left(k^{[\alpha]}, k^{[\beta]}\right) \log \frac{P\left(k^{[\alpha]}, k^{[\beta]}\right)}{P\left(k^{[\alpha]}\right), P\left(k^{[\beta]}\right)},
$$

where $P\left(k^{[\alpha]}, k^{[\beta]}\right)$ is the probability of finding a node degree $k^{[\alpha]}$ in a layer $\alpha$ and a degree $k^{[\beta]}$ in a layer $\beta$. The higher the value of $I_{\alpha, \beta}$, the more correlated (or anticorrelated) is the degree distribution of the two layers and, consequently, the structure of a time series associated with them. We also find the mean value of $I_{\alpha, \beta}$ for all possible pairs of layers - the scalar $\left\langle I_{\alpha, \beta}\right\rangle$ that quantifies the information flow in the system.

The quantity that quantitatively describes the distribution of a node degree $i$ between different layers is the entropy of a multiplexed degree:

$$
S_{i}=-\sum_{\alpha=1}^{M} \frac{k_{i}^{[\alpha]}}{o_{i}} \ln \frac{k_{i}^{[\alpha]}}{o_{i}} .
$$


Entropy is zero if all connected to $i$ edges are in the same layer, and has the maximum value when they are evenly distributed between different layers. So, the higher value of $S_{i}$, the more evenly distributed edges connected to $i$ between different layers.

A similar indicator is the multiplex participation coefficient:

$$
P_{i}=\frac{M}{M-1}\left[1-\sum_{\alpha=1}^{M}\left(\frac{k_{i}^{\alpha}}{o_{i}}\right)^{2}\right] .
$$

The $P_{i}$ takes values on the interval $[0,1]$ and determines how homogeneously are distributed the links of a node $i$ among $M$ layers. If a node is only active on one layer, $P_{i}=0 ; P_{i}=1$ if a node has an precisely defined number of incident links that are equally distributed across $M$ layers.

Obviously, measures $S_{i}$ and $P_{i}$ are very similar.

We will show that some of these spectral and topological indicators serve as the measures of system complexity, and the dynamic of their changes allow us to build precursors of crashes and critical events in the cryptocurrency market.

As far as multiplex measures are concerned, they are very similar in their dynamic to the spectral and topological representations above (see figure 28). In the case of a shorter sample of a base of three layers - Bitcoin, Ethereum, Litecoin (see figure 29-31), we have the asymmetric behavior of the multiplex measures $I, O, o$ (Equations (57), (54), (56) and $S, P$ (Equations (58) and (59)) for different methods of building multiplex networks.

Figures 29-31 show that the mentioned multiplex measures are excellent indicators that warn about the approaching crisis phenomenon, that is, are indicatorprecursors.

\section{Quantum precursors}

Quantum econophysics, a direction distinguished by the use of mathematical apparatus of quantum mechanics as well as its fundamental conceptual ideas and relativistic aspects, developed within its boundaries just a couple of years later, in the first decade of the $21^{\text {st }}$ century $[41,349$ 351].

According to classical physics, immediate values of physical quantities, which describe the system status, not only exist but can also be exactly measured. Although non-relativistic quantum mechanics does not reject the existence of immediate values of classic physical quantities, it postulates that not all of them can be measured simultaneously (Heisenberg uncertainty ratio). Relativistic quantum mechanics denies the existence of immediate values for all kinds of physical quantities, and, therefore, the notion of system status seizes to be algoristic.

In this section, we will demonstrate the possibilities of quantum econophysics on the example of the application of the Heisenberg uncertainty principle and the Random Matrices Theory to the actual and debatable now market of cryptocurrencies [80, 352].

\subsection{Heisenberg uncertainty principle and economic analogues of basic physical quantities}

In our paper [42], we have suggested a new paradigm of complex systems modeling based on the ideas of quantum as well as relativistic mechanics. It has been revealed that the use of quantum-mechanical analogies (such as the uncertainty principle, the notion of the operator, and quantum measurement interpretation) can be applied to describing socio-economic processes. Methodological and philosophical analysis of fundamental physical notions and constants, such as time, space, and spatial coordinates, mass, Planck's constant, light velocity from modern theoretical physics provides an opportunity to search for adequate and useful analogs in socio-economic phenomena and processes.

The Heisenberg uncertainty principle is one of the cornerstones of quantum mechanics. The modern version of the uncertainty principle, deals not with the precision of a measurement and the disturbance it introduces, but with the intrinsic uncertainty any quantum state must possess, regardless of what measurement is performed [353, 354]. Recently, the study of uncertainty relations, in general, has been a topic of growing interest, specifically in the setting of quantum information and quantum cryptography, where it is fundamental to the security of certain protocols $[355,356]$.

To demonstrate it, let us use the known Heisenberg's uncertainty ratio which is the fundamental consequence of non-relativistic quantum mechanics axioms and appears to be (e.g., [357]):

$$
\Delta x \cdot \Delta v \geqslant \frac{\hbar}{2 m_{0}},
$$

where $\Delta x$ and $\Delta v$ are mean square deviations of $x$ coordinate and velocity $v$ corresponding to the particle with (rest) mass $m_{0}, \hbar$ - Planck's constant. Considering values $\Delta x$ and $\Delta v$ to be measurable when their product reaches their minimum, according to equation (60) we derive:

$$
m_{0}=\frac{\hbar}{2 \cdot \Delta x \cdot \Delta v},
$$

i.e., the mass of the particle is conveyed via uncertainties of its coordinate and velocity - time derivative of the same coordinate.

Economic measurements are fundamentally relative, local in time, space and other socio-economic coordinates, and can be carried out via consequent and/or parallel comparisons "here and now", "here and there", "yesterday and today", "a year ago and now", etc.

Due to these reasons constant monitoring, analysis, and time series prediction (time series imply data derived from the dynamics of stock indices, exchange rates, cryptocurrency prices, spot prices, and other socio-economic indicators) become relevant for the evaluation of the state, tendencies, and perspectives of global, regional, and national economies.

Suppose there is a set of $K$ time series, each of $N$ samples, that correspond to the single distance $T$, with an 


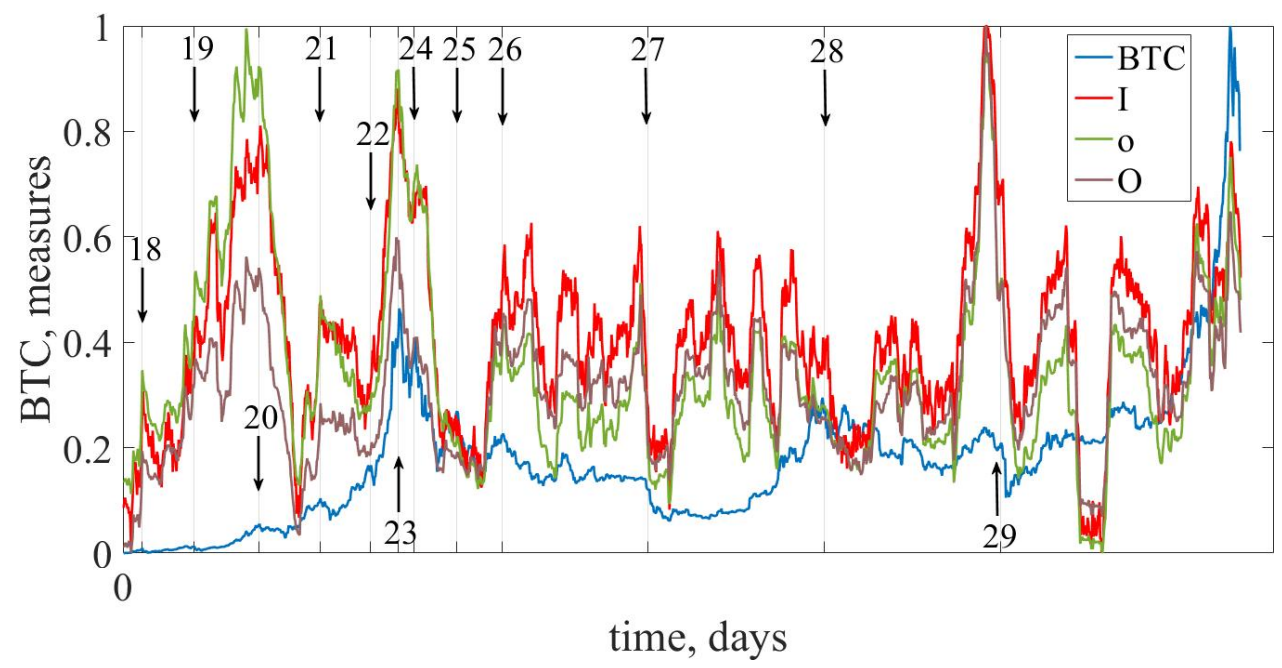

(a)

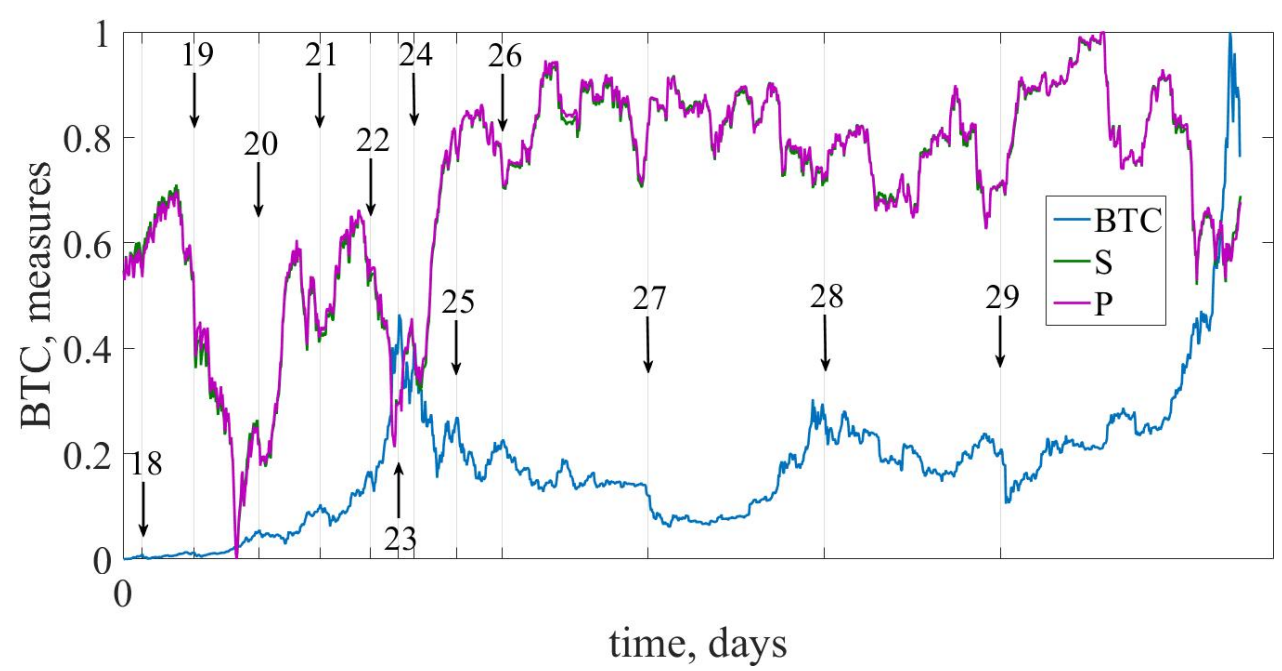

(b)

Figure 29: Dynamics of the second BTC period and multiplex measures for a base of three layers. The graph was built using the multiplex visibility graph

equally minimal time step $\Delta t_{\min }$ :

$\mathrm{X}_{i}\left(t_{n}\right), \quad$ for $n=0,1, \ldots, N-1$; for $i=1,2, \ldots, K$

To bring all series to the unified and non-dimensional representation, accurate to the additive constant, we normalize them, have taken a natural logarithm of each term of the series. Then, consider that every new series $\mathrm{X}_{i}\left(t_{n}\right)$ is a one-dimensional trajectory of a certain fictitious or abstract particle numbered $i$, while its coordinate is registered after every time $\operatorname{span} \Delta t_{\text {min }}$, and evaluate mean square deviations of its coordinate and speed in some time window $\Delta T=\Delta N \cdot \Delta t_{\text {min }}=\Delta N, 1 \ll \Delta N \ll N$. The "immediate" speed of $i^{\text {th }}$ particle at the moment $t_{n}$ is defined by the ratio:

$$
v_{i}\left(t_{n}\right)=\frac{x_{i}\left(t_{n+1}\right)-x_{i}\left(t_{n}\right)}{\Delta t_{\min }}=\frac{1}{\Delta t_{\min }} \ln \frac{\mathrm{X}_{i}\left(t_{n+1}\right)}{\mathrm{X}_{i}\left(t_{n}\right)}
$$

with variance $D_{v_{i}}$ and standard deviation $\Delta v_{i}$.
Keeping an analogy with equation (1), after some transformations, we can write an uncertainty ratio for this trajectory [42]:

$$
\frac{1}{\Delta t_{\min }}\left(\left\langle\ln ^{2} \frac{\mathrm{X}_{i}\left(t_{n+1}\right)}{\mathrm{X}_{i}\left(t_{n}\right)}\right\rangle_{n, \Delta N}-\left(\left\langle\ln ^{2} \frac{\mathrm{X}_{i}\left(t_{n+1}\right)}{\mathrm{X}_{i}\left(t_{n}\right)}\right\rangle_{n, \Delta N}\right)^{2}\right) \sim \frac{h}{m_{i}},
$$

where $m_{i}$ - economic "mass" of a $\mathrm{X}_{i}$ series, $h$ - value which comes as an economic Planck's constant.

Since the analogy with physical particle trajectory is merely formal, $h$ value, unlike the physical Planck's constant $\hbar$, can, generally speaking, depend on the historical period, for which the series are taken, and the length of the averaging interval (e.g., economical processes are different in the time of crisis and recession), on the series number $i$ etc. Whether this analogy is correct or not depends on the particular series' properties.

In recent research $[30,358]$, we tested the economic mass as an indicator of crisis phenomena on stock index 


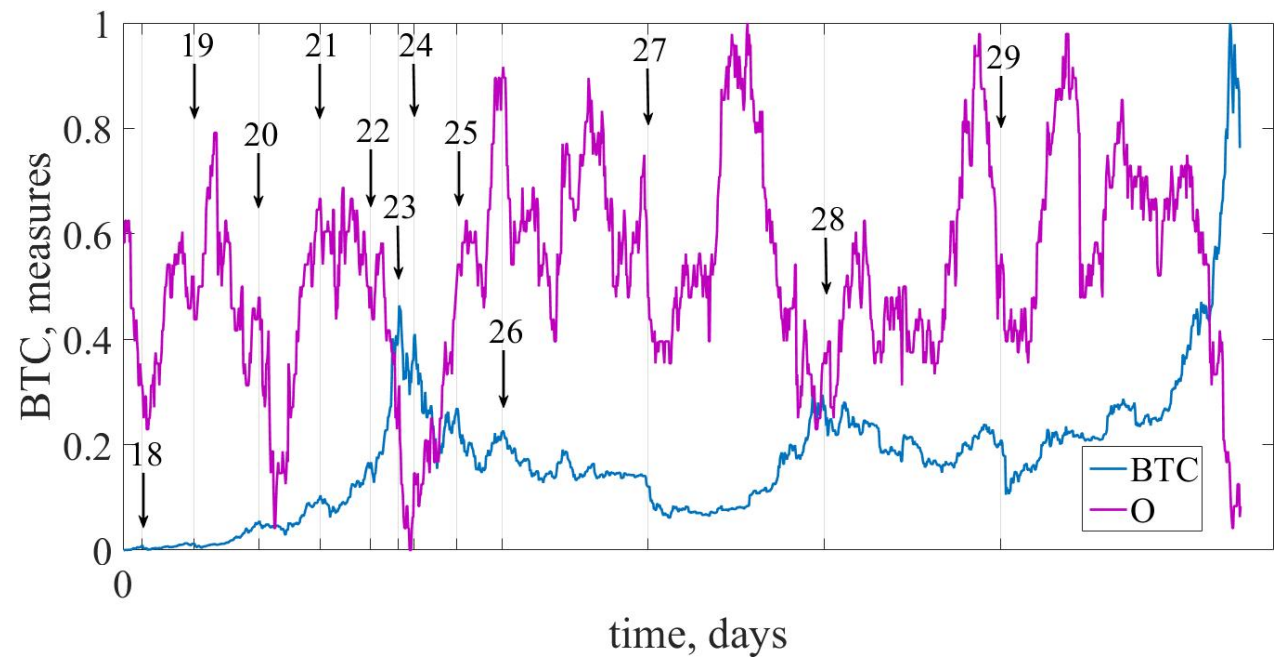

(a)

Figure 30: Dynamics of BTC daily prices and multiplex measures $O$. The graph was built using the MHVG

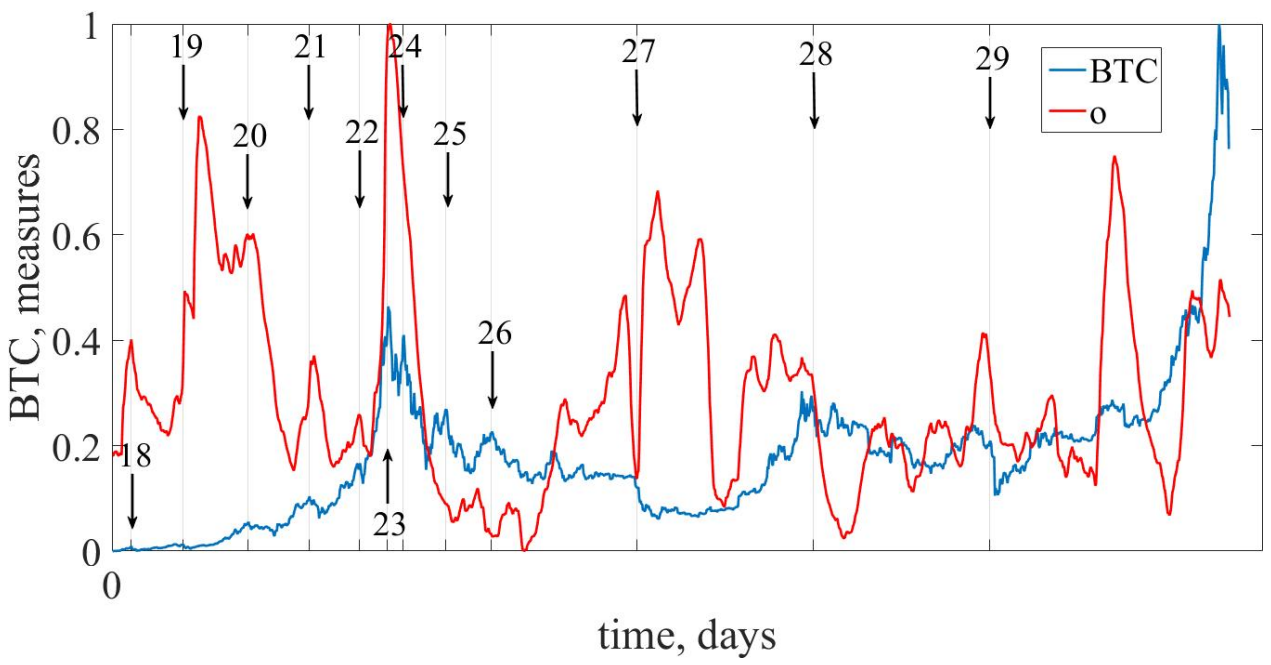

(a)

Figure 31: Dynamics of BTC daily prices and multiplex measures $o$. The graph was built using the MCRP

data. Here, we test the model for the cryptocurrency market on the example of Bitcoin [79, 80, 352, 359].

Obviously, there is a dynamic characteristic value $m$, depending on the internal dynamics of the market. In times of crashes and critical events marked by arrows, mass $m$ is significantly reduced in the pre-crash and pre-critical periods.

Obviously, $m$ remains a good indicator-precursor even in this case. Value $m$ is considerably reduced before a special market condition. The market becomes more volatile and prone to changes.

Next method of quantum econophysics is borrowed from nuclear physicists and is called Random Matrix Theory.

\subsection{Random matrix theory and quantum indicators-precursors}

Random Matrix Theory (RMT) developed in this context the energy levels of complex nuclei, which the existing models failed to explain (Wigner, Dyson, Mehta, and others $[353,354,356])$. Deviations from the universal predictions of RMT identify specific, nonrandom properties of the system under consideration, providing clues about the underlying interactions.

Unlike most physical systems, where one relates correlations between subunits to basic interactions, the underlying "interactions" for the stock market problem are not known. Here, we analyze cross-correlations between stocks by applying concepts and methods of random matrix theory, developed in the context of complex quantum 


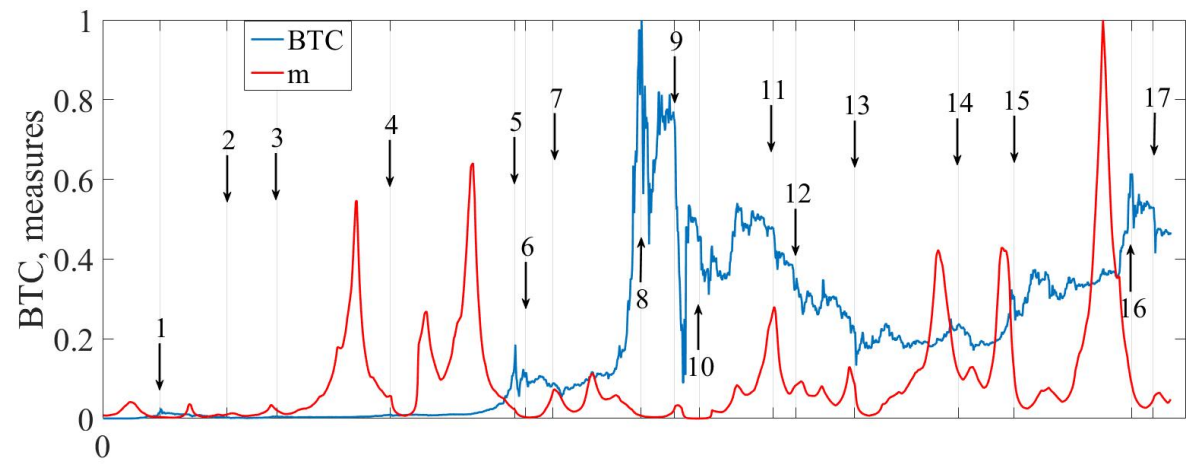

time, days

(a)

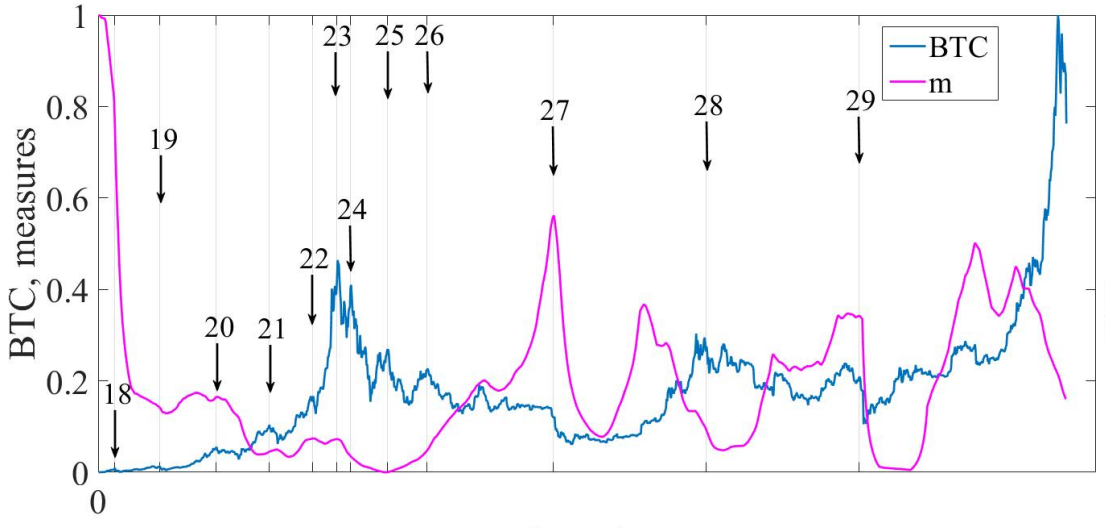

time, days

(b)

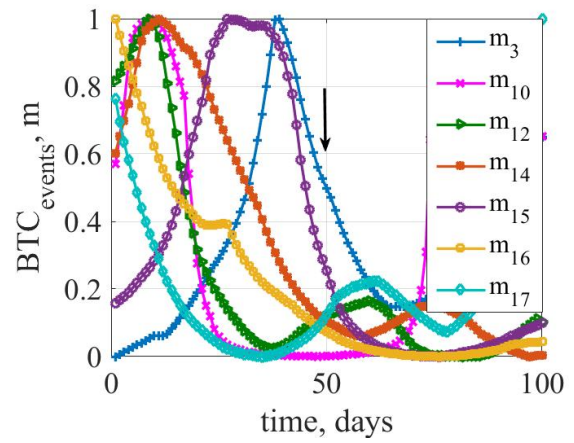

(d)

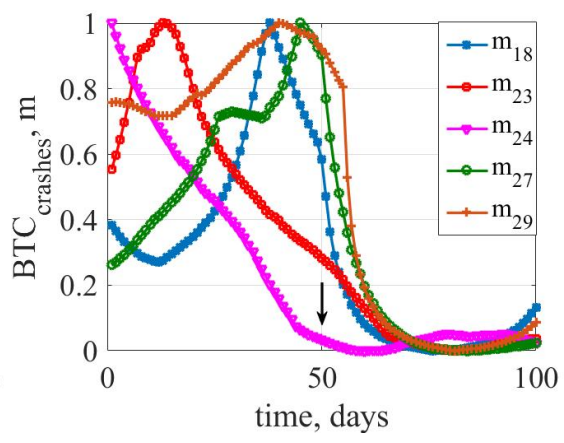

(e)

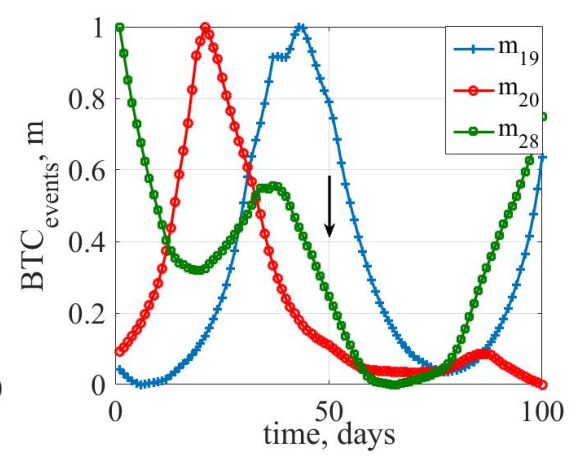

(f)

Figure 32: Dynamics of measure $m$ for two entire periods, and its dynamics for their local crashes (c, e) and critical events $(d, f)$ with the window size of 50 days and step of 1 day

systems where the precise nature of the interactions between subunits are not known.

RMT has been applied extensively in studying multiple financial time series [42, 352, 355, 357-362].

Special databases have been prepared, consisting of cryptocurrency time series for a certain time. The largest number of cryptocurrencies 689 contained a base of 456 days from 31.12.2017 to 31.05 .2020 , and the smallest (22 cryptocurrencies) contained a base, respectively, from 11.09.2013 to 31.05.2020 (https://coinmarketcap.com/all/ views/all/). In order to quantify correlations, we first cal- culate the logarithmic returns of the cryptocurrencies price series over a time scale $\Delta t=1$ day. We calculate the pairwise cross-correlation coefficients between any two cryptocurrency time series returns. for the largest database, a graphical representation of the pair correlation field is shown in figure 33a. For comparison, a map of correlations of randomly mixed time series of the same length shown in figure $33 \mathrm{~b}$.

For the correlation matrix $C$ we can calculate its eigenvalues, $C=U \Lambda U^{T}$, where $U$ denotes the eigenvectors, $\Lambda$ is the eigenvalues of the correlation matrix, whose density 


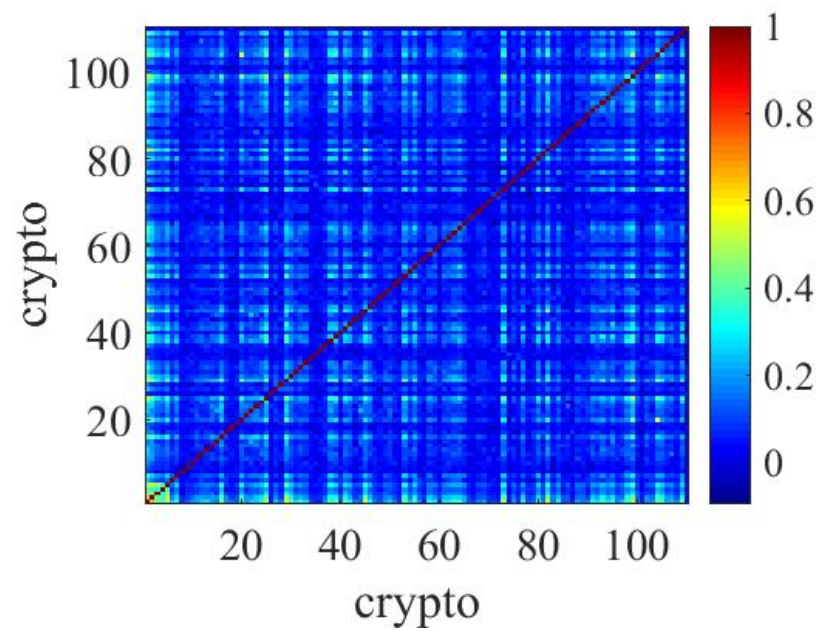

(a)

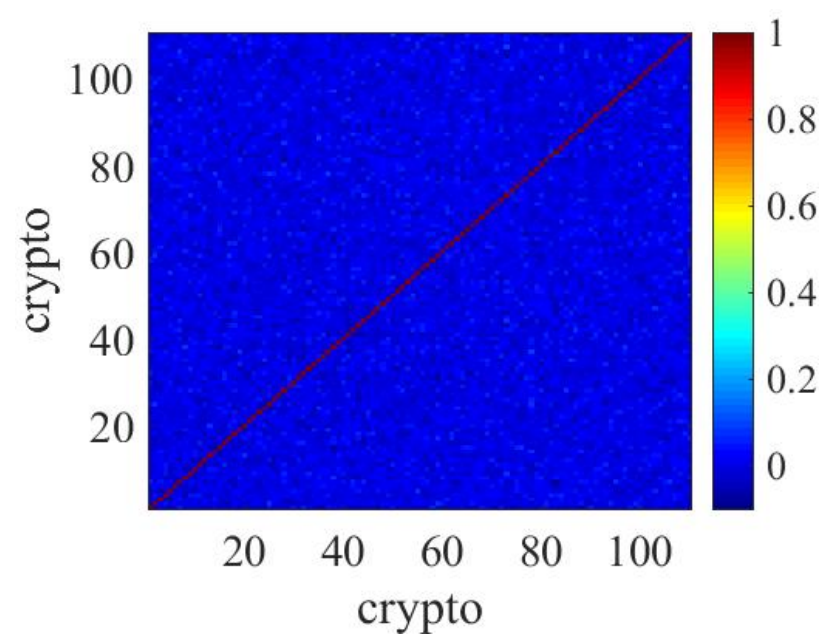

(b)

Figure 33: Visualization of the field of correlations for the initial (a, c) and mixed (b, d) matrix cryptocurrency

$f_{c}(\lambda)$ is defined as $f_{c}(\lambda)=(1 / N) d n(\lambda) / d \lambda$, where $n(\lambda)$ is the number of eigenvalues of $C$ that are less than $\lambda$. In the limit $N \rightarrow \infty, T \rightarrow \infty$ and $Q=T / N \geqslant 1$ fixed, the probability density function $f_{c}(\lambda)$ of eigenvalues $\lambda$ of the random correlation matrix $M$ has a closed form:

$$
f_{c}(\lambda)=\frac{Q}{2 \pi \sigma^{2}} \frac{\sqrt{\left(\lambda_{\max }-\lambda\right)\left(\lambda-\lambda_{\min }\right)}}{\lambda},
$$

with $\lambda \in\left[\lambda_{\min }, \lambda_{\text {max }}\right]$, where $\lambda_{\text {min }}^{\max }$ is given by $\lambda_{\text {min }}^{\max }=\sigma^{2}(1+$ $1 / Q \pm 2 \sqrt{1 / Q}$ ) and $\sigma^{2}$ is equal to the variance of the elements of matrix $M$.

We compute the eigenvalues of the correlation matrix $C, \lambda_{\max }=\lambda_{1}>\lambda_{2}>\cdots>\lambda_{15}=\lambda_{\text {min }}$. The probability density functions of paired correlation coefficients $c_{i j}$ and eigenvalues $\lambda_{i}$ for matrices of 110,298 , and 689 cryptocurrencies are presented in figure 34 .

From figure 34 it can be seen that the distribution functions for the paired correlation coefficients of the selected matrices differ significantly from the distribution function described by the RMT. It can be seen that the crypto mar-

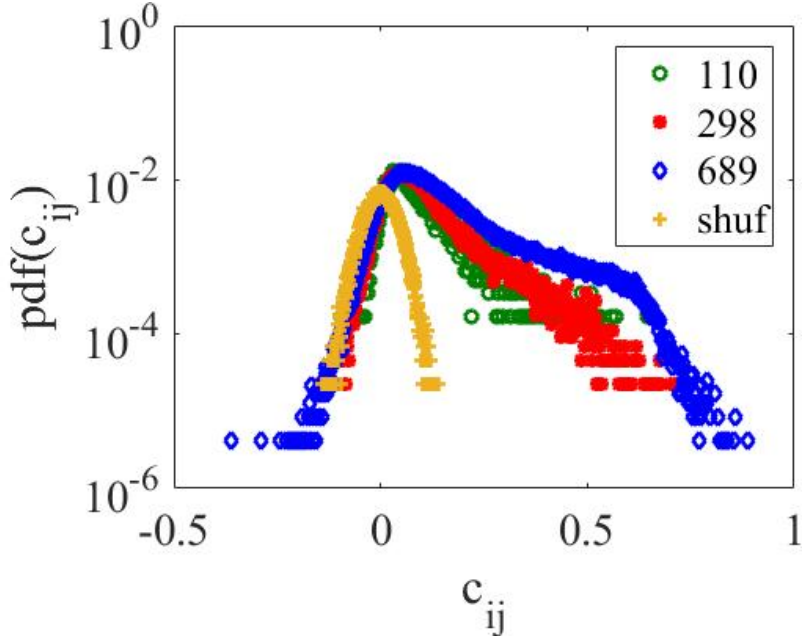

(a)

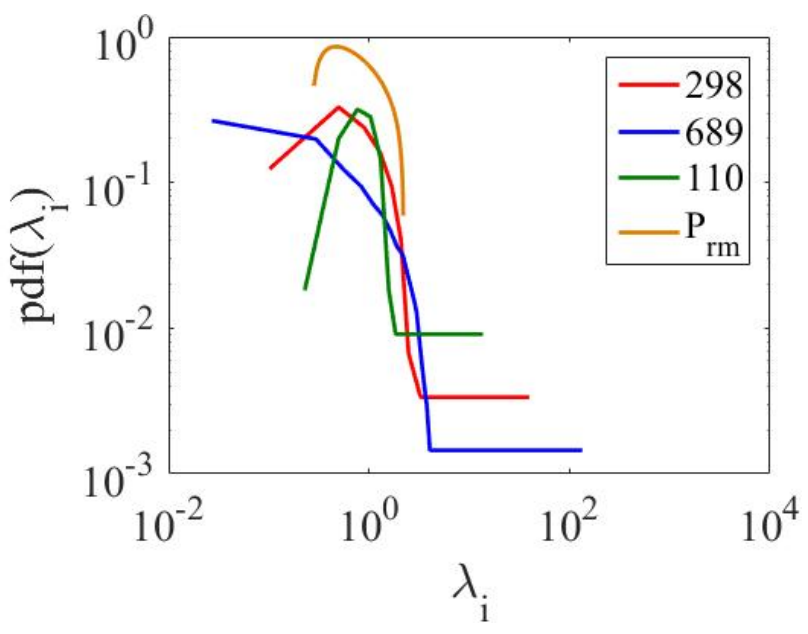

(b)

Figure 34: Comparison of distributions of the pair correlation coefficients (a) and eigenvalues of the correlation matrix (b) obtained for initial cryptocurrencies and their shuffled version

ket has a significantly correlated, self-organized system, and the difference from the RMT of the case, the correlation coefficients exceed the value of $0.6-0.8$ on "thick tails". The distribution of eigenvalues of the correlation matrix also differs markedly from the case of RMT. In our case, only one-third of its values refer to the RMT region.

Eigenvectors correspond to the participation ratio PR and its inverse participation ratio IPR

$$
I^{k}=\sum_{l=1}^{N}\left[u_{l}^{l}\right]^{4}
$$

where $u_{k}^{l}, l=1, \ldots, N$, are the components of the eigenvector $u^{k}$. So, PR indicates the number of eigenvector components that contribute significantly to that eigenvector. More specifically, a low IPR indicates that cryptocurrencies contribute more equally. In contrast, a large IPR would imply that the factor is driven by the dynamics of a small number of cryptocurrencies. 
The irregularity of the influence of eigenvalues of the correlation matrix is determined by the absorption ratio (AR)

$$
A R_{n}=\frac{\sum_{k=1}^{n} \lambda_{l}}{\sum_{k=1}^{N} \lambda_{k}},
$$

which is a cumulative risk measure and indicates which part of the overall variation is described from the total number of $N$ eigenvalues.

The difference in dynamics is due to the peculiarities of non-random correlations between the time series of individual assets. Under the framework of RMT, if the eigenvalues of the real-time series differ from the prediction of RMT, there must exist hidden economic information in those deviating eigenvalues. For cryptocurrencies markets, there are several deviating eigenvalues in which the largest eigenvalue $\lambda_{\max }$ reflects a collective effect of the whole market. As for PR, the differences from RMT appear at large and small $\lambda$ values and are similar to the Anderson quantum effect of localization [363]. Under crash and critical event conditions, the states at the edges of the distributions of eigenvalues are delocalized, thus identifying the beginning of one of these events. This is evidenced by the results presented in figure $35 \mathrm{~b}$.

We find that both $\lambda_{\max }$ and $P R \lambda_{\max }$ have large values for periods containing the crypto market crashes and critical events. At the same time, their growth begins in the pre-crashes periods. As well as the economic mass, they are quantum precursors of crashes and critical events phenomena.

\section{Conclusions}

Definitely, the factors from within and outside of the cryptocurrencies universe are going to evolve all of them. The great influence will go from incumbents and policymakers, as well as challengers and users. Current mistrust on the part of the government may lead to the introduction of specific licensing requirements that may make these digital currencies less attractive. Similarly, the adaptation to them and acceptance of cryptocurrencies may lead to increasing demand for them. The current situation with coronavirus is of paramount importance and is of significant danger.

From the literature overview, we have understood that crashes and critical events do not disappear without a trace, but will also affect the fate of individuals. On the other hand, in the future, the influence of these events may attract users to alternative forms of currency such as cryptocurrencies. Increased trading activity on cryptocurrency exchanges could positively affect the popularity of stablecoins. Lastly, the overall monetary system may be fundamentally changed through the introduction of a central bank digital currency, potentially upstaging stablecoins [364].

In order to give reliable, powerful, and simple indicators-precursors that are able to minimize further losses as a result of changes, we addressed the reach arsenal of the theory of complexity and the methods of nonlinear dynamics that can identify special trajectories in the complex dynamics and classify them. Following our

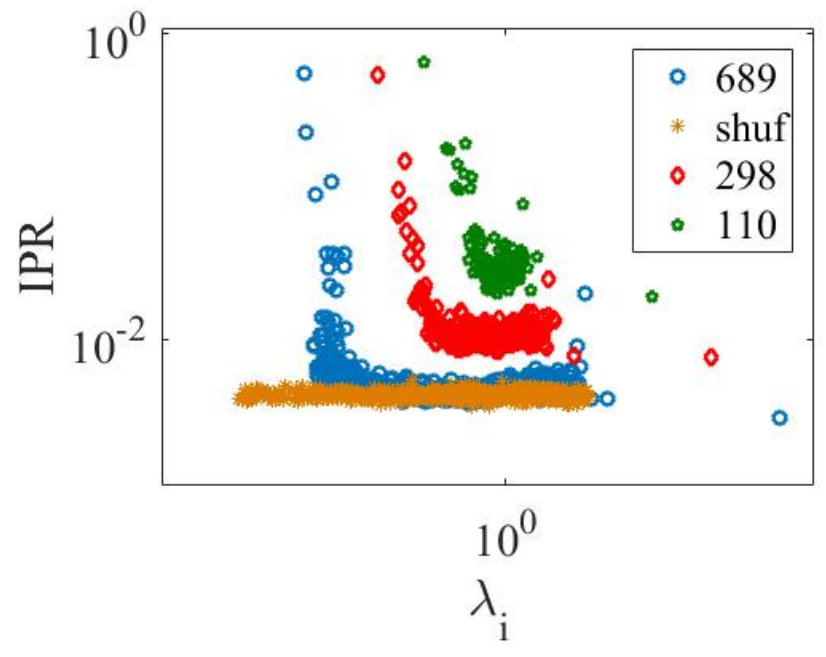

(a)

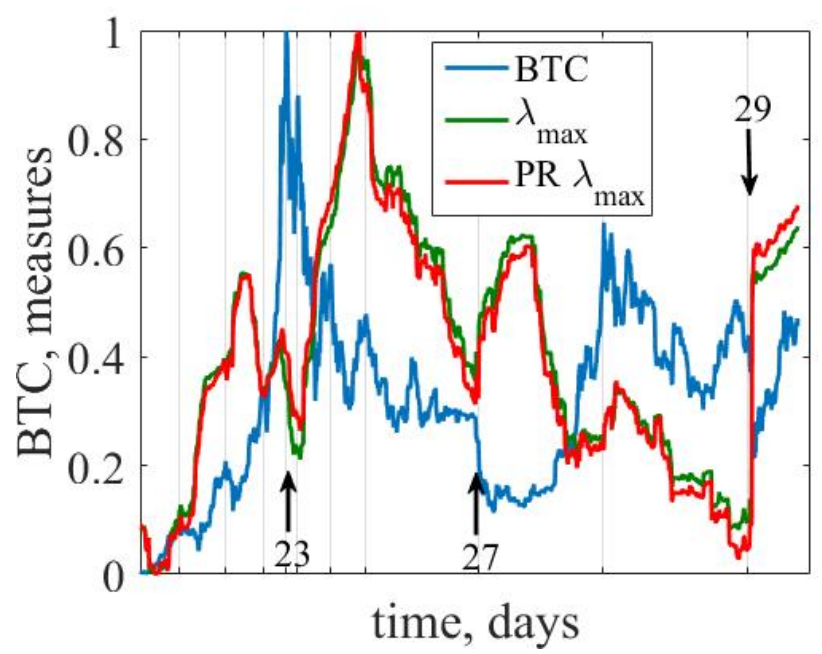

(b)

Figure 35: Inverse participation ratio (a) and (b) quantum measures of complexity $\lambda_{\max }$ and its participation ratio

research, we obtain informational, (multi-)fractal, chaosdynamical, recurrent, irreversible, based on complex networks, and quantum measures of complexity.

The obtained quantitative methods were applied to classified crashes of the Bitcoin market, where it was seen that these indicators can be used in order to protect yourself from the upcoming critical change. To draw some conclusions about its evolution and factors that influence it, we pointed out the most influential critical changes in this market. Relying on different research articles and our previous experience, such changes were classified as crashes and critical events. Moreover, we assume crash as strong, time localized drop with high volatility whose percentage decline exceeds $30 \%$. Critical events are those falls with less percentage decline and volatility. The analysis of the crypto market with the sliding (rolling or moving) window approach allowed us to draw some conclusions about its evolution and factors that influence it. Regarding empirical results, we have shown that some of the measures 
are very sensitive to the length of the sliding window and its time step. For example, if we consider two closest to each other events, a previous event that had much more volatility can have a great influence on the corresponding measure of complexity and spoil the identification of the next less influential, but important event. Thus, time localization is significant while calculating the measure of complexity. The less time localization and time step, the more corresponding changes are taken into account. For a much larger time window and its step, we can have less accurate estimations.

It turned out that most of the chosen measures of complexity respond in advance to the corresponding changes of complexity in the cryptocurrency market and can be used in the diagnostic processes. Such measures can be presented as indicators or even indicators-precursors of the approaching crashes and critical events.

Relying on the information theory and its powerful toolkit, we emphasized four measures of complexity, such as the measure of Lempel-Ziv, classical Shannon entropy, and its two modifications (Approximate entropy and Permutation entropy). We referred to the complexity of the systems, how it was described in different studies, and what methods were applied to quantify its degree. Our results show that in the pre-crash or pre-critical period, the complexity of Bitcoin starts to change that is it starts to decrease, indicating that such events presented to be more predictable and corresponding patterns are more structured. Thus, the degree of predictability increases in times of such events.

Along with information theory, we referred to the multifractal properties of the cryptocurrency market. As it was obtained with multifractal detrended fluctuation analysis, the scaling exponents remain non-linear, and the width of singularity spectrum changes in time that gives evidence that at different times (scales) BTC time series exhibits more or less complex behavior, indicating that cryptocurrency exhibits multifractal properties. Applying the width of multifractality as an indicator of possible critical states we found that before crash or critical event, this measure starts to decrease that tell us that the series is expected to be more predictable and stable, while its dynamics after such events is increasing that present system to be more susceptible to fluctuations.

Chaos-dynamical measures were applied to study bitcoin from the perspective of Chaos theory. Transitions between chaotic and non-chaotic behavior were identified with Lyapunov exponents where there was selected the largest Lyapunov exponent. The beginning of each crash or critical event can be characterized by the convergence of the two considered, initially close trajectories where the Largest Lyapunov exponent reflects the rate of such convergence, while the high-price level regimes are characterized by the divergence of the initially close trajectories. Whereas decreasing largest Lyapunov exponent indicates that patterns in the system tend to be predictable (trajectories converge), increasing indicates that the system goes into an unstable regime. Our empirical results obtained with the sliding window presented the largest Lyapunov exponent to be an indicator of such regimes. Moreover, we considered that such extreme events can be related to the fat-tails and better described with non-Gaussian distributions, particularly, described by Lévy alpha-stable distribution and its four parameters. As it is still debatable whether the stable distribution is completely applicable or not, we addressed to its group of stable parameters, and during tests, we emphasized that the characteristic exponent that serve to describe the thickness of tails is the best for serving as an indicator-precursor of possible crashes and critical events. Thus, is shown that such a complex system as the bitcoin market, with growth and preferential attachments, is characterized by power-laws.

The analysis of the crypto market with the measures from the recurrence quantification analysis revealed that its toolkit is suitable for distinguishing diverse market periods. Such measures as recurrence rate, determinism, the entropy diagonal line histogram, recurrence periodic density entropy, recurrence entropy, and divergence, which methodology is based on clusters of isolated points, vertical/diagonal lines, etc., are presented to be great for detection of the periods of instability or relaxation.

Also, applying the concept of time irreversibility (asymmetry), we found it changes for the period of crashes and critical events, and corresponding measures detect such phenomena. Thus, time irreversibility based on permutation patterns can serve as a good base for further models of financial control. However, much more measures can be added to this list [107, 316, 365-367].

Moreover, we have demonstrated the possibility of studying complex cryptosystems within the network paradigm. The time series can be presented as an economic network (visibility graph) and multiplex network with a set of both spectral and topological characteristics, which are sensitive to the critical changes in the BTC market.

Addressing to quantum econophysics and its apparatus where appropriate measures of complexity were obtained. Such quantitative methods as Heisenberg uncertainty and the Random matrix approach have confirmed their effectiveness for studying the cryptocurrency market. We found that economic "mass" along with $\lambda_{\max }$ and $P R \lambda_{\max }$ are presented to be effective due to their robustness, computational efficiency, and simplicity.

Apparently, the impact of different crashes and critical events was reflected in the cryptocurrency market, as well as the coronavirus pandemic and therefore, the dynamics of past events, as well as of the subsequent could be identified in advance using the appropriate indicators of the theory of complexity. In our further studies, we are going to aim our view on exploring and analyzing of other methods from the theory of complexity. Being emerging currency that still needs to become trustworthy among as many people as possible, bitcoin's dynamics is the subject not only to high fluctuations but also to various attacks [368-372] of blockchain [373] and Proof-of-Work protocol [374]. Thus, particular interest presents research on implementing the theory of complex systems and its enormous toolkit for identifying such abnormal activity when transactions between users happen to make appropriate actions in advance. Equally interesting is the development 
of the theory of quantum computation and quantum information [375-378] where it would be interesting to see their influence on blockchain and cryptocurrencies. Moreover, the research in the field of artificial intelligence, machine, and deep learning does not remain without attention [51, 379-390].

\section{References}

[1] R. Albert, A.L. Barabási, Rev. Mod. Phys. 74, 47 (2002)

[2] C. Reuven, H. Shlomo, Complex Networks: Structure, Robustness and Function (Cambridge University Press, 2010), ISBN 9780521841566

[3] M.E.J. Newman, SIAM Review 45, 167-256 (2003)

[4] M. Newman, A.L. Barabasi, D.J. Watts, eds., The Structure and Dynamics of Networks (Princeton University Press, Princeton, NJ, USA, 2006)

[5] G. Nicolis, I. Prigogine, Exploring complexity: an introduction (W.H. Freeman, 1989), ISBN 9780716718598

[6] A. Rai, A. Mahata, M. Nurujjaman, O. Prakash, Statistical properties of the aftershocks of stock market crashes: evidence based on the 1987 crash, 2008 financial crisis and COVID-19 pandemic (2020), arXiv:2012.03012 [q-fin.ST]

[7] E. Mnif, A. Jarboui, K. Mouakhar, Finance Research Letters 36, 101647 (2020)

[8] A. Ammy-Driss, M. Garcin, Efficiency of the financial markets during the COVID-19 crisis: timevarying parameters of fractional stable dynamics (2020), arXiv:2007.10727 [q-fin.ST]

[9] R. Cerqueti, V. Ficcadenti, Anxiety for the pandemic and trust in financial markets (2020), arXiv:2008.01649 [q-fin.ST]

[10] M. Costola, M. Iacopini, C.R.M.A. Santagiustina, Public Concern and the Financial Markets during the COVID-19 outbreak (2020), arXiv:2005.06796 [q-fin.ST]

[11] M. Feldkircher, F. Huber, M. Pfarrhofer, Measuring the Effectiveness of US Monetary Policy during the COVID-19 Recession (2020), arXiv: 2007. 15419 [econ. EM]

[12] M. Garcin, J. Klein, S. Laaribi, Estimation of timevarying kernel densities and chronology of the impact of COVID-19 on financial markets (2020), arXiv:2007.09043 [q-fin.ST]

[13] M. Pagano, C. Wagner, J. Zechner, Disaster resilience and asset prices (2020), arXiv:2005.08929 [q-fin.GN]

[14] A.A. Toda, Susceptible-Infected-Recovered (SIR) Dynamics of COVID-19 and Economic Impact (2020), arXiv:2003.11221 [q-bio.PE]

[15] S. Drożdż, J. Kwapień, P. Oświę, T. Stanisz, M. Wa̧torek, Entropy 22 (2020)

[16] R.K.K. Pang, O. Granados, H. Chhajer, E.F. Legara, An analysis of network filtering methods to sovereign bond yields during COVID-19 (2021), arXiv:2009.13390 [q-fin.ST]

[17] S. Semerikov, S. Chukharev, S. Sakhno, A. Striuk, V. Osadchyi, V. Solovieva, T. Vakaliuk, P. Nechypurenko, O. Bondarenko, H. Danylchuk, E3S Web of Conferences 166 (2020)

[18] H. Danylchuk, L. Kibalnyk, O. Kovtun, A. Kiv, O. Pursky, G. Berezhna, CEUR Workshop Proceedings 2713, 352 (2020)

[19] A. Kaminskyi, M. Nehrey, N. Rizun, CEUR Workshop Proceedings 2713, 204 (2020)

[20] N. Maksyshko, O. Vasylieva, I. Kozin, V. Perepelitsa, CEUR Workshop Proceedings 2713, 219 (2020)

[21] S. Semerikov, H. Kucherova, V. Los, D. Ocheretin, CEUR Workshop Proceedings 2845, 22 (2021)

[22] G. Malinetsky, Modeling and Analysis of Information Systems 19, 5 (2015)

[23] V. Soloviev, N. Moiseienko, O. Tarasova, CEUR Workshop Proceedings 2393, 905 (2019)

[24] S. Somin, Y. Altshuler, G. Gordon, A. Pentland, E. Shmueli, Scientific Reports 10, 4587 (2020)

[25] P. Grau, C. Jaureguizar, D. Jaureguizar, Esic Market Economics and Business Journal 49, 569 (2018)

[26] J. Liang, L. Li, D. Zeng, PLOS ONE 13, 1 (2018)

[27] S. Thurner, P. Klimek, R. Hanel, Introduction to the theory of complex systems (Oxford University Press, Oxford, 2018)

[28] S. Drożdż, L. Minati, P. Oświęcimka, M. Stanuszek, M. Wa̧torek, Chaos: An Interdisciplinary Journal of Nonlinear Science 30, 023122 (2020)

[29] M. Wa̧torek, S. Drożdż, J. Kwapień, L. Minati, P. Oświęcimka, M. Stanuszek, Physics Reports 901, 1 (2021), multiscale characteristics of the emerging global cryptocurrency market

[30] A.O. Bielinskyi, I. Khvostina, A. Mamanazarov, A. Matviychuk, S. Semerikov, O. Serdyuk, V. Solovieva, V.N. Soloviev, IOP Conference Series: Earth and Environmental Science 628, 012019 (2021)

[31] M. Ausloos, D. Grech, T.D. Matteo, R. Kutner, C. Schinckus, H.E. Stanley, Physica A: Statistical Mechanics and its Applications 531, 136 (2020)

[32] W.B. Arthur, Nature Reviews Physics 3, 136 (2021)

[33] R. Kutner, M. Ausloos, D. Grech, T. Di Matteo, C. Schinckus, H. Eugene Stanley, Physica A: Statistical Mechanics and its Applications 516, 240 (2019)

[34] R. Mantegna, H. Stanley, An Introduction to Econophysics: Correlations and Complexity in Finance, Vol. 53 (2000)

[35] Z.Q. Jiang, W.J. Xie, W.X. Zhou, D. Sornette, Reports on Progress in Physics 82, 125901 (2019)

[36] J. Kwapień, S. Drożdż, Physics Reports 515, 115 (2012), physical approach to complex systems

[37] B.E. Baaquie, Quantum Finance: Path Integrals and Hamiltonians for Options and Interest Rates 
(Cambridge University Press, 2004)

[38] M. Schaden, Physica A: Statistical Mechanics and its Applications 316, 511-538 (2002)

[39] V.P. Maslov, V.E. Nazaikinskii, Mathematics underlying the 2008 financial crisis, and a possible remedy (2009), arXiv:0811.4678 [q-fin.GN]

[40] C. Schinckus, A Methodological Call for a Quantum Econophysics, in Selected Papers of the 7th International Conference on Quantum Interaction Volume 8369 (Springer-Verlag, Berlin, Heidelberg, 2013), QI 2013, p. 308-316

[41] V. Saptsin, V. Soloviev, Relativistic quantum econophysics - new paradigms in complex systems modelling (2009), arXiv:0907.1142 [physics.soc-ph]

[42] V. Soloviev, V. Saptsin, Heisenberg uncertainty principle and economic analogues of basic physical quantities (2011), arXiv: 1111.5289 [physics.gen-ph]

[43] D. Sornette, Why Stock Markets Crash: Critical Events in Complex Financial Systems (Princeton University Press, 2003)

[44] Y. Peng, P. Albuquerque, J. Camboim de Sá, A.J. Padula, M. Montenegro, Expert Systems with Applications 97 (2017)

[45] Q. Zhao, A deep learning framework for predicting digital asset price movement from trade-by-trade data (2020), arXiv:2010.07404 [q-fin.ST]

[46] M. Amjad, D. Shah, Trading Bitcoin and Online Time Series Prediction, in Proceedings of the Time Series Workshop at NIPS 2016, edited by O. Anava, A. Khaleghi, M. Cuturi, V. Kuznetsov, A. Rakhlin (PMLR, Barcelona, Spain, 2017), Vol. 55 of Proceedings of Machine Learning Research

[47] T. Chen, C. Guestrin, Proceedings of the 22nd ACM SIGKDD International Conference on Knowledge Discovery and Data Mining (2016)

[48] V. Derbentsev, A. Matviychuk, V. Soloviev, Forecasting of Cryptocurrency Prices Using Machine Learning (Springer, 2020), pp. 211-231, 1st edn., ISBN 978-981-15-4498-9

[49] A.H. Dyhrberg, Finance Research Letters 16, 85 (2016)

[50] M. Ortu, N. Uras, C. Conversano, G. Destefanis, S. Bartolucci, On technical trading and social media indicators in cryptocurrencies' price classification through deep learning (2021), arXiv:2102.08189 [q-fin.ST]

[51] N. Uras, L. Marchesi, M. Marchesi, R. Tonelli, Forecasting bitcoin closing price series using linear regression and neural networks models (2020), arXiv:2001.01127 [q-fin.ST]

[52] A. Hachicha, F. Hachicha, Review of Quantitative Finance and Accounting 56, 647 (2021)

[53] J. Kaminski, Nowcasting the bitcoin market with twitter signals (2016), arXiv: 1406.7577 [CS.SI]
[54] M.A. Kennis, Multi-channel discourse as an indicator for bitcoin price and volume movements (2018), arXiv: 1811.03146 [q-fin.ST]

[55] O. Kodama, L. Pichl, T. Kaizoji, CBU International Conference Proceedings 5, 384 (2017)

[56] D. Shah, K. Zhang, Bayesian regression and bitcoin (2014), arXiv: 1410.1231 [CS.AI]

[57] V. Soloviev, S. V., in Modern problems of forecasting socio-economic processes: concepts, models, applied aspects (Tkachuk O. V., 2012), pp. 174188

[58] M. Tarnopolski, Modeling the price of bitcoin with geometric fractional brownian motion: a monte carlo approach (2017), arXiv: 1707.03746 [q-fin. CP]

[59] N. Courtois, M. Grajek, R. Naik, Optimizing SHA256 in Bitcoin Mining (2014), Vol. 448 of Communications in Computer and Information Science, pp. 131-144, ISBN 978-3-662-44892-2

[60] L. Kristoufek, Frontiers in Physics 8 (2020)

[61] D. Broomhead, G.P. King, Physica D: Nonlinear Phenomena 20, 217 (1986)

[62] V.I. Ponomarenko, M.D. Prokhorov, Phys. Rev. E 66, 026215 (2002)

[63] M. Rajković, Physica A: Statistical Mechanics and its Applications 287, 383 (2000)

[64] G.M. Caporale, L.A. Gil-Alana, A. Plastun, CESifo Working Paper Series 6811, CESifo (2017), https://ideas.repec.org/p/ces/ceswps/ 6811.html

[65] A.F. Bariviera, M.J. Basgall, W. Hasperué, M. Naiouf, Physica A: Statistical Mechanics and its Applications 484, 82-90 (2017)

[66] A.F. Bariviera, Economics Letters 161, 1-4 (2017)

[67] J. Wang, G. Meric, Z. Liu, I. Meric, Journal of Banking \& Finance 33, 1563 (2009)

[68] S. Lleo, W.T. Ziemba, Financial Markets, Institutions \& Instruments 26, 61 (2017)

[69] H. Hong, J. Stein, Review of Financial Studies 16, 487 (2003)

[70] M. Shu, W. Zhu, Physica A: Statistical Mechanics and its Applications 548, 124477 (2020)

[71] T. Klein, H. Pham Thu, T. Walther, International Review of Financial Analysis 59, 105 (2018)

[72] K. Gkillas, F. Longin, SSRN Electronic Journal pp. 1-85 (2019)

[73] Y. Liu, A. Tsyvinski, X. Wu, NBER Working Papers 25882, National Bureau of Economic Research, Inc (2019)

[74] L. Kristoufek, PLOS ONE 10, 1 (2015)

[75] X. Li, C. Wang, Decision Support Systems 95, 49 (2017)

[76] A. Bielinskyi, V. Soloviev, S. Semerikov, V. Solovieva, CEUR Workshop Proceedings 2422, 420 (2019)

[77] A. Bielinskyi, S. Semerikov, V. Solovieva, V. Soloviev, SHS Web Conf. 65, 06006 (2019) 
[78] V. Derbentsev, S. Semerikov, O. Serdyuk, V. Solovieva, V. Soloviev, E3S Web Conf. 166, 13031 (2020)

[79] V. Soloviev, A. Belinskiy, Complex Systems Theory and Crashes of Cryptocurrency Market (Springer, Cham, 2019), Vol. 1007 of ICTERI 2018, pp. 276297, ISBN 978-3-030-13928-5

[80] V. Soloviev, A. Belinskiy, CEUR Workshop Proceedings 2104, 116 (2018)

[81] V. Soloviev, A. Bielinskyi, O. Serdyuk, V. Solovieva, S. Semerikov, CEUR Workshop Proceedings 2732, 455 (2020)

[82] V. Soloviev, S. Yevtushenko, V. Batareyev, CEUR Workshop Proceedings 2393, 434 (2019)

[83] V. Soloviev, V. Solovieva, A. Tuliakova, M. Ivanova, Construction of crisis precursors in multiplex networks, in Proceedings of the 2019 7th International Conference on Modeling, Development and Strategic Management of Economic System (MDSMES 2019) (Atlantis Press, 2019/10), pp. 361-366, ISBN 978-94-6252-800-0, ISSN 2352-5428, https: //doi .org/10.2991/mdsmes-19.2019.68

[84] V. Soloviev, O. Serdiuk, S. Semerikov, O. KohutFerens, Recurrence entropy and financial crashes, in Proceedings of the 2019 7th International Conference on Modeling, Development and Strategic Management of Economic System (MDSMES 2019) (Atlantis Press, 2019/10), pp. 385-388, ISBN 97894-6252-800-0, ISSN 2352-5428, https://doi. org/10.2991/mdsmes-19.2019.73

[85] V. Soloviev, A. Bielinskyi, N. Kharadzjan, CEUR Workshop Proceedings 2832, 24 (2020)

[86] M.S. Kanwal, J.A. Grochow, N. Ay, Entropy 19 (2017)

[87] D. Bonchev, Information Theoretic Complexity Measures (Springer, 2009), pp. 4820-4838

[88] L. Lovász, The Emergence of Complexity in Mathematics, Physics, Chemistry and Biology, Pontifical Academy of Sciences p. 12 (1996)

[89] L.T. Lui, G. Terrazas, H. Zenil, C. Alexander, N. Krasnogor, Artificial Life 21, 205 (2015)

[90] C.E. Shannon, Bell System Technical Journal 27, 379 (1948)

[91] G. Sommazzi, Ph.D. thesis (2016)

[92] J.L. Blanc, L. Pezard, A. Lesne, Phys. Rev. E 84, 036214 (2011)

[93] S. Zozor, P. Ravier, O. Buttelli, Physica A: Statistical Mechanics and its Applications 345, 285 (2005)

[94] E. Estevez-Rams, R. Lora Serrano, B. Aragón Fernández, I. Brito Reyes, Chaos: An Interdisciplinary Journal of Nonlinear Science 23, 023118 (2013)

[95] S.D. Silva, R. Matsushita, R. Giglio, Economics Bulletin 7, 1 (2008)

[96] R. Giglio, R. Matsushita, A. Figueiredo, I. Gleria, S.D. Silva, EPL (Europhysics Letters) 84, 48005 (2008)
[97] S. Da Silva, MPRA Paper 64497, University Library of Munich, Germany (2015)

[98] S. Da Silva, C. Taufemback, R. Giglio, Economics Bulletin 31, 1631 (2011)

[99] R. Giglio, S. Da Silva, MPRA Paper 22720, University Library of Munich, Germany (2009)

[100] A. Lempel, J. Ziv, IEEE Transactions on Information Theory 22, 75 (1976)

[101] O. Brandouy, J.P. Delahaye, L. Ma, H. Zenil, Research in International Business and Finance 30, 336 (2014)

[102] P. Fiedor, Risks 3, 219 (2015)

[103] J. Gao, Y. Hou, F. Fan, F. Liu, Entropy 22 (2020)

[104] H. Cao, Y. Li, Chaos: An Interdisciplinary Journal of Nonlinear Science 24, 013134 (2014)

[105] V. Soloviev, S. Semerikov, V. Solovieva, LempelZiv Complexity and Crises of Cryptocurrency Market, in Proceedings of the III International Scientific Congress Society of Ambient Intelligence 2020 (ISC-SAI 2020) (Atlantis Press, 2020), pp. 299-306, ISBN 978-94-6252-933-5, ISSN 2352-5428, https://doi.org/10.2991/ aebmr.k.200318.037

[106] A.N. Kolmogorov, International Journal of Computer Mathematics 2, 157 (1968)

[107] M. Costa, C.K. Peng, A. Goldberger, Cardiovascular engineering (Dordrecht, Netherlands) 8, 88 (2008)

[108] R. Clausius, T. Hirst, The Mechanical Theory of Heat: With Its Applications to the Steam-Engine and to the Physical Properties of Bodies (Creative Media Partners, LLC, 2017)

[109] L. Boltzmann, Weitere Studien über das Wärmegleichgewicht unter Gasmolekülen (Vieweg+Teubner Verlag, 1970), Vol. 67, pp. 115-225

[110] J.W. Gibbs, Elementary principles in statistical mechanics developed with especial reference to the rational foundation of thermodynamics (C. Scribner, New York, 1902)

[111] M. Vosvrda, J. Barunik, L. Vacha, M. Vošvrda, Czech Economic Review 4, 281 (2010)

[112] J.S. Richman, J.R. Moorman, American Journal of Physiology-Heart and Circulatory Physiology 278, H2039 (2000)

[113] R. Sole, S. Valverde, Information Theory of Complex Networks: On Evolution and Architectural Constraints (Springer, 2004), Vol. 207, pp. 189207, ISBN 978-3-540-22354-2

[114] V. Soloviev, O. Serdiuk, in Information technologies and modeling in economics: on the way to interdisciplinarity (Gate-Ukraine, 2013), pp. 115129

[115] C. Tsallis, Introduction to nonextensive statistical mechanics: Approaching a complex world (Springer, 2009), ISBN 978-0-387-85358-1

[116] A. Delgado-Bonal, A. Marshak, Entropy 21 (2019)

[117] S. Pincus, Proceedings of the National Academy of Sciences of the United States of America 88, 


$$
\text { 2297-2301 (1991) }
$$

[118] S.M. Pincus, A.L. Goldberger, American Journal of Physiology-Heart and Circulatory Physiology 266, H1643 (1994)

[119] K. Yun, H.K. Park, D.H. Kwon, Y.T. Kim, S.N. Cho, H.J. Cho, B.S. Peterson, J. Jeong, Psychiatry Research: Neuroimaging 201, 226 (2012)

[120] S.N. Bhaduri, Journal of Emerging Market Finance 13, 43 (2014)

[121] C. Eom, G. Oh, W.S. Jung, Physica A: Statistical Mechanics and its Applications 387, 5511 (2008)

[122] S. Lahmiri, S. Bekiros, Chaos, Solitons \& Fractals 138, 109936 (2020)

[123] I. Mahmoud, K. Naoui, H. Jemmali, International Journal of Economics and Financial Issues 3, 683 (2013)

[124] S. Pincus, R.E. Kalman, Proceedings of the National Academy of Sciences 101, 13709 (2004)

[125] W.Q. Duan, H.E. Stanley, Phys. Rev. E 81, 066116 (2010)

[126] A. Delgado-Bonal, Sci. Rep. 9, 2045 (2019)

[127] C. Bandt, B. Pompe, Phys. Rev. Lett. 88, 174102 (2002)

[128] J. Amigó, Permutation Complexity in Dynamical Systems (Springer-Verlag Berlin Heidelberg, 2010)

[129] M. Zanin, L. Zunino, O.A. Rosso, D. Papo, Entropy 14, 1553 (2012)

[130] H. Kantz, T. Schreiber, Nonlinear Time Series Analysis, 2nd edn. (Cambridge University Press, 2003)

[131] M. Henry, G. Judge, Econometrics 7 (2019)

[132] H. Sigaki, M. Perc, H. Ribeiro, Scientific Reports 9 (2019)

[133] A. Sensoy, Finance Research Letters 28, 68 (2019)

[134] A. Metin Karakaş, Asian Journal of Business and Management 7, 10 (2019)

[135] D.T. Pele, M. Mazurencu-Marinescu-Pele, Entropy 21 (2019)

[136] F. Takens, in Dynamical Systems and Turbulence, Warwick 1980, edited by D. Rand, L.S. Young (Springer, Berlin, 1981), Vol. 898 of Lecture Notes in Mathematics, chap. 21, pp. 366-381

[137] J.P. Eckmann, D. Ruelle, Rev. Mod. Phys. 57, 617 (1985)

[138] E. Ott, T. Sauer, J. Yorke, Coping with Chaos, Wiley Series in Nonlinear Science (Wiley, 1994)

[139] C. Webber, Jr., J. Zbilut, in Tutorials in Contemporary Nonlinear Methods for the Behavioral Sciences, edited by M.A. Riley, G.C.V. Orden (National Science Foundation (NSF), 2005), chap. 2

[140] R. Gu, Physica A: Statistical Mechanics and its Applications 484, 215 (2017)

[141] B.B. Mandelbrot, J.A. Wheeler, American Journal of Physics 51, 286 (1983)

[142] H.E. Hurst, Transactions of the American Society of Civil Engineers 116, 770 (1951)

[143] H.E. Hurst, Nature 180, 494 (1957)
[144] A.W. Lo, Working Paper 2984, National Bureau of Economic Research (1989)

[145] C.K. Peng, S.V. Buldyrev, S. Havlin, M. Simons, H.E. Stanley, A.L. Goldberger, Phys. Rev. E 49, 1685 (1994)

[146] Z.Q. Jiang, W.J. Xie, W.X. Zhou, Physica A: Statistical Mechanics and its Applications 405, 235 (2014)

[147] J.W. Kantelhardt, S.A. Zschiegner, E. KoscielnyBunde, S. Havlin, A. Bunde, H. Stanley, Physica A: Statistical Mechanics and its Applications 316, 87-114 (2002)

[148] F. Aslam, W. Mohti, P. Ferreira, International Journal of Financial Studies 8 (2020)

[149] R. Hasan, S.M. Mohammad, Physica A: Statistical Mechanics and its Applications 419, 746 (2015)

[150] S. Kumar, N. Deo, Physica A: Statistical Mechanics and its Applications 388, 1593 (2009)

[151] J. Kwapień, P. Oświęcimka, S. Drożdż, Physica A: Statistical Mechanics and its Applications 350, 466 (2005)

[152] S. Lahmiri, Physica A: Statistical Mechanics and its Applications 486, 183 (2017)

[153] J.W. Lee, K. Eun Lee, P. Arne Rikvold, Physica A: Statistical Mechanics and its Applications 364, 355 (2006)

[154] K. Matia, Y. Ashkenazy, H.E. Stanley, Europhysics Letters (EPL) 61, 422 (2003)

[155] P. Suárez-García, D. Gómez-Ullate, Physica A: Statistical Mechanics and its Applications 394, 226 (2014)

[156] L. Zunino, A. Figliola, B.M. Tabak, D.G. Pérez, M. Garavaglia, O.A. Rosso, Chaos, Solitons \& Fractals 41, 2331 (2009)

[157] F. Delbianco, F. Tohmé, T. Stosic, B. Stosic, Physica A: Statistical Mechanics and its Applications 457, 573 (2016)

[158] R. Gu, H. Chen, Y. Wang, Physica A: Statistical Mechanics and its Applications 389, 2805 (2010)

[159] Z. Li, X. Lu, Energy Procedia 5, 1920 (2011), 2010 International Conference on Energy, Environment and Development - ICEED2010

[160] P. Mali, A. Mukhopadhyay, Physica A: Statistical Mechanics and its Applications 413, 361-372 (2014)

[161] S. Zheng, X. Lan, Physica A: Statistical Mechanics and its Applications 444, 547 (2016)

[162] G. Lim, S. Kim, H. Lee, K. Kim, D.I. Lee, Physica A: Statistical Mechanics and its Applications 386, 259 (2007)

[163] P. Caraiani, E. Haven, Physica A Statistical Mechanics and its Applications 419, 395 (2015)

[164] P. Norouzzadeh, B. Rahmani, Physica A: Statistical Mechanics and its Applications 367, 328 (2006)

[165] G. Oh, C. Eom, S. Havlin, W.S. Jung, F. Wang, H. Stanley, S. Kim, Eur. Phys. J. B 85, 214 (2012)

[166] J. Qin, X. Lu, Y. Zhou, L. Qu, Physica A: Statistical Mechanics and its Applications 421, 443 (2015) 
[167] D.H. Wang, X.W. Yu, Y.Y. Suo, Physica A: Statistical Mechanics and its Applications 391, 3503 (2012)

[168] P. Norouzzadeh, W. Dullaert, B. Rahmani, Physica A: Statistical Mechanics and its Applications 380, 333 (2007)

[169] W. Mensi, A.K. Tiwari, S.M. Yoon, Physica A: Statistical Mechanics and its Applications 471, 135 (2017)

[170] A.K. Tiwari, C.T. Albulescu, S.M. Yoon, Physica A: Statistical Mechanics and its Applications 483, 182 (2017)

[171] Y. Wang, Y. Wei, C. Wu, Physica A: Statistical Mechanics and its Applications 390, 817 (2011)

[172] L. Zunino, B. Tabak, A. Figliola, D. Pérez, M. Garavaglia, O. Rosso, Physica A: Statistical Mechanics and its Applications 387, 6558 (2008)

[173] B. Podobnik, H.E. Stanley, Phys. Rev. Lett. 100, 084102 (2008)

[174] W.X. Zhou, Physical Review E 77 (2008)

[175] N. Costa, C. Silva, P. Ferreira, International Journal of Financial Studies 7 (2019)

[176] X.Y. Qian, Y.M. Liu, Z.Q. Jiang, B. Podobnik, W.X. Zhou, H.E. Stanley, Phys. Rev. E 91, 062816 (2015)

[177] Z.Q. Jiang, W.X. Zhou, Phys. Rev. E 84, 016106 (2011)

[178] L. Kristoufek, EPL (Europhysics Letters) 95, 68001 (2011)

[179] J. Wang, P. Shang, W. Ge, Fractals 20, 271 (2012)

[180] J. Li, X. Lu, Y. Zhou, Physica A: Statistical Mechanics and its Applications 453, 131 (2016)

[181] C. Xie, Y. Zhou, G. Wang, X. Yan, Fluctuation and Noise Letters 16, 1750004-226 (2017)

[182] F. Ma, Y. Wei, D. Huang, Physica A: Statistical Mechanics and its Applications 392, 1659 (2013)

[183] Y. Wang, Y. Wei, C. Wu, Physica A: Statistical Mechanics and its Applications 389, 5468 (2010)

[184] P. Yue, H.C. Xu, W. Chen, X. Xiong, W.X. Zhou, Fractals 25, 1750041 (2017)

[185] F. Ma, Y. Wei, D. Huang, L. Zhao, Physica A: Statistical Mechanics and its Applications 392, 5356 (2013)

[186] F. Ma, Q. Zhang, C. Peng, Y. Wei, Physica A: Statistical Mechanics and its Applications 410, 154 (2014)

[187] Y. Wang, Y. Wei, C. Wu, Physica A: Statistical Mechanics and its Applications 390, 864 (2011)

[188] X. Zhuang, Y. Wei, F. Ma, Physica A: Statistical Mechanics and its Applications 430, 101 (2015)

[189] X. Zhuang, Y. Wei, B. Zhang, Physica A: Statistical Mechanics and its Applications 399, 113 (2014)

[190] L. Xinsheng, L. Jianfeng, Z. Ying, Q. Yubo, Physica A: Statistical Mechanics and its Applications 486, 168 (2017)

[191] Z. Zhang, Y. Zhang, D. Shen, W. Zhang, Complexity 2018, 1 (2018)
[192] Z. Zhang, Y. Zhang, D. Shen, W. Zhang, Physica A: Statistical Mechanics and its Applications 508, 67 (2018)

[193] Z. Da, J. Engelberg, P. Gao, Review of Financial Studies 28, 1 (2015)

[194] W. Zhang, P. Wang, X. Li, D. Shen, Journal of Behavioral and Experimental Finance 18, 50 (2018)

[195] M. Gronwald, C. Sattarhof, Working Paper 5, University of Aberdeen Business School (2018)

[196] T. Takaishi, Physica A: Statistical Mechanics and its Applications 506, 507-519 (2018)

[197] L. Kirichenko, V. Bulakh, T. Radivilova, 2017 4th International Scientific-Practical Conference Problems of Infocommunications. Science and Technology (PIC S\&T) (2017)

[198] Y. Jiang, H. Nie, W. Ruan, Finance Research Letters 25, 280 (2018)

[199] A. Sensoy, E. Hacihasanoglu, Energy Economics 46, 318 (2014)

[200] K.H. Al-Yahyaee, W. Mensi, S.M. Yoon, Finance Research Letters 27, 228 (2018)

[201] G. Gajardo, W.D. Kristjanpoller, M. Minutolo, Chaos, Solitons \& Fractals 109, 195 (2018)

[202] S. Lahmiri, S. Bekiros, A. Salvi, Chaos, Solitons \& Fractals 107, 43 (2018)

[203] W. Zhang, P. Wang, X. Li, D. Shen, Complexity 2018, 1 (2018)

[204] A. Ganchuk, V. Derbentsev, V. Soloviev, Multifractal Properties of the Ukraine Stock Market (2006), arXiv:physics/0608009v1 [physics.data-an]

[205] K. Hu, P.C. Ivanov, Z. Chen, P. Carpena, H. Eugene Stanley, Phys. Rev. E 64, 011114 (2001)

[206] Z. Chen, P.C. Ivanov, K. Hu, H.E. Stanley, Phys. Rev. E 65, 041107 (2002)

[207] J.R. Thompson, J.R. Wilson, Mathematics and Computers in Simulation 126, 63 (2016)

[208] D. Clark, L. Tarra, A. Berera, Phys. Rev. Fluids 5, 064608 (2020)

[209] R. Engelken, F. Wolf, L.F. Abbott, Lyapunov spectra of chaotic recurrent neural networks (2020), arXiv:2006.02427 [nlin.CD]

[210] K. Krishnamurthy, T. Can, D.J. Schwab, Theory of gating in recurrent neural networks (2021), arXiv:2007.14823 [cond-mat.dis-nn]

[211] W.S. Lee, S. Flach, Deep learning of chaos classification (2020), arXiv: 2004.10980 [cs.LG]

[212] M.B. Tayel, E.I. AlSaba, Robust and sensitive method of lyapunov exponent for heart rate variability (2015), arXiv: 1508.00996 [CS.SY]

[213] H. De Thélin, T. Gauthier, G. Vigny, Bulletin of the London Mathematical Society (2020)

[214] M. Cencini, F. Cecconi, A. Vulpiani, Chaos: From Simple Models to Complex Systems, Vol. 17 of Series on Advances in Statistical Mechanics (2010)

[215] P. Grassberger, I. Procaccia, Phys. Rev. Lett. 50, 346 (1983) 
[216] J.C. Sprott, Chaos and Time-Series Analysis (Princeton University Press, 2001), ISBN 9780198508403

[217] L.S. Young, Journal of Physics A: Mathematical and Theoretical 46, 254001 (2013)

[218] C.J. Gavilán-Moreno, G. Espinosa-Paredes, Nuclear Engineering and Technology 48, 434 (2016)

[219] A. Prieto-Guerrero, G. Espinosa-Paredes, Dynamics of BWRs and mathematical models (2019), pp. $193-268$

[220] D. Nychka, S. Ellner, A.R. Gallant, D. McCaffrey, Journal of the Royal Statistical Society: Series B (Methodological) 54, 399 (1992)

[221] A. Wolf, J. Swift, H.L. Swinney, J. Vastano, Physica D: Nonlinear Phenomena 16, 285 (1985)

[222] M. Sano, Y. Sawada, Phys. Rev. Lett. 55, 1082 (1985)

[223] J.P. Eckmann, S.O. Kamphorst, D. Ruelle, S. Ciliberto, Phys. Rev. A 34, 4971 (1986)

[224] M.T. Rosenstein, J.J. Collins, C.J. De Luca, Physica D: Nonlinear Phenomena 65, 117 (1993)

[225] U. Parlitz, International Journal of Bifurcation and Chaos 02, 155 (1992)

[226] M. Balcerzak, D. Pikunov, A. Dabrowski, Nonlinear Dynamics 94, 3053 (2018)

[227] J. Gao, Y. Cao, W.W. Tung, J. Hu, Multiscale Analysis of Complex Time Series: Integration of Chaos and Random Fractal Theory, and Beyond (Wiley, 2007), ISBN 978-0-470-19164-4

[228] J. Gao, J. Hu, W.W. Tung, Y. Zheng, Quantitative Finance 13, 265 (2013)

[229] V. Soloviev, D. Chabanenko, S. I., Using the scaledependent Lyapunov exponent as a measure of complexity, in The banking system of Ukraine in the context of globalization of financial markets: proceedings of VII International scientific and practical conference, edited by M. Gedz (CHIBS UBS NBU, 2012), pp. 469-471

[230] V. Soloviev, I. Stratiychuk, The problems of economics 2, 279 (2013)

[231] J.P. Eckmann, S.O. Kamphorst, D. Ruelle, Europhysics Letters (EPL) 4, 973 (1987)

[232] J. Scheinkman, B. Lebaron, The Journal of Business 62, 311 (1989)

[233] H.D.I. Abarbanel, R. Brown, J.J. Sidorowich, L.S. Tsimring, Rev. Mod. Phys. 65, 1331 (1993)

[234] V.S. Kulkarni, Complexity, chaos, and the duffingoscillator model: An analysis of inventory fluctuations in markets (2013), arXiv:1308.1616 [q-fin.GN]

[235] O. Bajo-Rubio, F. Fernandez-Rodriguez, S. Sosvilla-Rivero, Economics Letters 39, 207 (1992)

[236] W.D. Dechert, R. Gencay, Journal of Applied Econometrics 7, S41 (1992)

[237] R. Gençay, Physica D: Nonlinear Phenomena 89, 261 (1996)
[238] S. Shreemoyee, C. Vikhyat, Engineering and Technology International Journal of Economics and Management Engineering 13 (2019)

[239] S. Srinivasan, S. Prasad, S. Patil, G. Lazarou, J. Picone, Estimation of Lyapunov Spectra from a Time Series, in Proceedings of the IEEE SoutheastCon 2006 (2006), pp. 192-195

[240] L. Mastroeni, P. Vellucci, "Chaos" in energy and commodity markets: a controversial matter (2017), arXiv: 1611.07432 [q-fin.ST]

[241] V. Plakandaras, R. Gupta, M.E. Wohar, Applied Economics 51, 4477 (2019)

[242] G. Chakrabarti, C. Sen, Anatomy of Global Stock Market Crashes, Number 978-81-322-0463-3 in SpringerBriefs in Economics (Springer, 2012)

[243] J. Liesen, V. Mehrmann, Linear Algebra, Springer Undergraduate Mathematics Series, 1st edn. (Springer, 2015)

[244] C. Touzé, A. Chaigne, Acustica 86, 1 (2000)

[245] B. Podobnik, A. Valentinčič, D. Horvatić, H.E. Stanley, Proceedings of the National Academy of Sciences of the United States of America 108, 17883-17888 (2011)

[246] L. Bachelier, Annales scientifiques de l'École Normale Supérieure 3e série, 17, 21 (1900)

[247] X. Gabaix, P. Gopikrishnan, V. Plerou, H. Stanley, Nature 423, 267 (2003)

[248] M. Kateregga, S. Mataramvura, D. Taylor, Cogent Economics \& Finance 5, 1318813 (2017)

[249] D. Krężołek, Studia Ekonomiczne 247, 57-68 (2015)

[250] T. Lux, D. Sornette, Journal of Money, Credit and Banking 34, 589 (2002)

[251] Y. Malevergne, V. Pisarenko, D. Sornette, Quantitative Finance 5, 379 (2005)

[252] Y. Malevergne, V. Pisarenko, D. Sornette, Phys. Rev. E 83, 036111 (2011)

[253] N.N. Taleb, International Journal of Forecasting 36, 1228-1240 (2020)

[254] N.N. Taleb, Y. Bar-Yam, P. Cirillo, On single point forecasts for fat-tailed variables (2020), arXiv:2007.16096 [physics.soc-ph]

[255] P. Gopikrishnan, M. Meyer, L. Amaral, H. Stanley, The European Physical Journal B 3, 139-140 (1998)

[256] P. Gopikrishnan, V. Plerou, L.A. Nunes Amaral, M. Meyer, H.E. Stanley, Phys. Rev. E 60, 5305 (1999)

[257] B. Podobnik, D. Horvatic, A.M. Petersen, H.E. Stanley, Proceedings of the National Academy of Sciences of the United States of America 106, 22079-22084 (2009)

[258] B. Podobnik, K. Matia, A. Chessa, P.C. Ivanov, Y. Lee, H.E. Stanley, Physica A Statistical Mechanics and its Applications 300, 300 (2001)

[259] X. Gabaix, Annual Review of Economics 1, 255 (2009) 
[260] Z. Kostanjčar, B. Jeren, Advances in Complex Systems 16, 1350008 (2013)

[261] A. Chakraborty, S. Easwaran, S. Sinha, Deviations from universality in the fluctuation behavior of a heterogeneous complex system reveal intrinsic properties of components: The case of the international currency market (2018), arXiv: 1606.06111 [q-fin.ST]

[262] T. Takaishi, Recent scaling properties of bitcoin price returns (2020), arXiv:2009.06874 [q-fin.ST]

[263] S. Drożdż, R. Gȩbarowski, L. Minati, P. Oświęcimka, M. Waa̧torek, Chaos: An Interdisciplinary Journal of Nonlinear Science 28, 071101 (2018)

[264] T. Takaishi, PLOS ONE 16, e0246209 (2021)

[265] S. Begušić, Z. Kostanjčar, H. Eugene Stanley, B. Podobnik, Physica A: Statistical Mechanics and its Applications 510, 400 (2018)

[266] B. Mandelbrot, The Journal of Business 36 (1963)

[267] P. Levy, Bulletin de la Société Mathématique de France 52, 49 (1924)

[268] B. Mandelbrot, International Economic Review 1, 79 (1960)

[269] E.S. Andersen, Mathematica Scandinavica 3, 185 (1955)

[270] I.A. Koutrouvelis, Journal of the American Statistical Association 75, 918 (1980)

[271] B. Brorsen, S. Yang, Communications in Statistics Part B: Simulation and Computation 19, 1459 (1990)

[272] J. Nolan, Maximum Likelihood Estimation and Diagnostics for Stable Distributions (Birkhäuser, Boston, MA, 2001), pp. 379-400

[273] E.F. Fama, R. Roll, Journal of the American Statistical Association 66, 331 (1971)

[274] J.H. McCulloch, Communications in Statistics Simulation and Computation 15, 1109 (1986)

[275] X. Ma, C.L. Nikias, IEEE Transactions on Signal Processing 43, 2884 (1995)

[276] M. Shao, C.L. Nikias, Proceedings of the IEEE 81, 986 (1993)

[277] J.M. Nicolas, Traitement Du Signal 19, 139 (2002)

[278] E.E. Kuruoglu, IEEE Transactions on Signal Processing 49, 2192 (2001)

[279] W.H. DuMouchel, The Annals of Statistics 1, 948 (1973)

[280] V.M. Zolotarev, One-dimensional stable distributions, Vol. 65 of Translations of Mathematical Monographs (American Mathematical Society, Providence, RI, 1986), translated from the Russian by H. H. McFaden, Translation edited by Ben Silver

[281] J.M. Chambers, C.L. Mallows, B.W. Stuck, Journal of the American Statistical Association 71, 340 (1976)

[282] S. Bianchi, A. Pantanella, International Journal of Trade, Economics and Finance 2, 52 (2011)
[283] V.I. Arnold, A. Avez, ZAMM - Journal of Applied Mathematics and Mechanics / Zeitschrift für Angewandte Mathematik und Mechanik 50, 506 (1970)

[284] K. Umeno, Nonlinear Theory and Its Applications, IEICE 7, 14 (2016)

[285] I.A. Koutrouvelis, Communications in Statistics Simulation and Computation 10, 17 (1981)

[286] V. Soloviev, V. Solovieva, D. Chabanenko, in Contemporary concepts of forecasting the development of complex socio-economic systems, edited by O. Chernyak, P. Zakharchenko (FO-P Tkachuk O V, Berdyansk, 2014), pp. 257-264

[287] H. Poincaré, The Three-Body Problem and the Equations of Dynamics, Astrophysics and Space Science Library, 1st edn. (Springer, Cham, 2017)

[288] P. Faure, H. Korn, Physica D: Nonlinear Phenomena 122, 265 (1998)

[289] M. Thiel, M. Romano, J. Kurths, Analytical Description of Recurrence Plots of White Noise and Chaotic Processes, in Applied Nonlinear Dynamics (2003), Vol. 11

[290] M. Thiel, M. Romano, J. Kurths, R. Meucci, E. Allaria, F. Arecchi, Physica D: Nonlinear Phenomena 171, 138 (2002)

[291] L. Charles, J. Webber, I. Cornel, M. Norbert, eds., Recurrence Plots and Their Quantifications: Expanding Horizons, Vol. 180 of Springer Proceedings in Physics (Springer, 2015)

[292] N. Marwan, M. Carmen Romano, M. Thiel, J. Kurths, Physics Reports 438, 237 (2007)

[293] G. Jianbo, C. Huaqing, Physics Letters A 270, 75 (2000)

[294] N. Marwan, N. Wessel, U. Meyerfeldt, A. Schirdewan, J. Kurths, Phys. Rev. E 66, 026702 (2002)

[295] C.L. Webber, J.P. Zbilut, Journal of Applied Physiology 76, 965 (1994)

[296] J.P. Zbilut, C.L. Webber, Physics Letters A 171, 199 (1992)

[297] G. Corso, T.L. Prado, G.Z. dos S. Lima, S.R. Lopes, A novel entropy recurrence quantification analysis (2017), arXiv: 1707.00944 [stat.0T]

[298] M.A. Little, P.E. McSharry, S.J. Roberts, D.A. Costello, I.M. Moroz, BioMedical Engineering OnLine 6, 23 (2007)

[299] H. Rabarimanantsoa, L. Achour, C. Letellier, A. Cuvelier, J.F. Muir, Chaos: An Interdisciplinary Journal of Nonlinear Science 17, 013115 (2007)

[300] S. Lopes, T. Prado, G. Corso, G. dos S. Lima, J. Kurths, Chaos, Solitons \& Fractals 133, 109616 (2020)

[301] A.L. Goldberger, L.A.N. Amaral, L. Glass, J.M. Hausdorff, P.C. Ivanov, R.G. Mark, J.E. Mietus, G.B. Moody, C.K. Peng, H.E. Stanley, Circulation 101, e215 (2000)

[302] M. Kirchner, P. Schubert, M. Liebherr, C.T. Haas, PLOS ONE 9, 1 (2014)

[303] I. Prigogine, From Being to Becoming Time and Complexity in the Physical Sciences, 1st edn. (W.H. 
Freeman \& Co, 1981)

[304] M. Costa, A.L. Goldberger, C.K. Peng, Phys. Rev. E 71, 021906 (2005)

[305] V. Soloviev, O. Rybchinska, Bulletin of the Kryvyi Rih Economic Institute KEI 2, 52 (2010)

[306] S. Daw, C. Finney, M. Kennel, Physical review. E, Statistical physics, plasmas, fluids, and related interdisciplinary topics 62, 1912 (2000)

[307] C. Diks, J. van Houwelingen, F. Takens, J. DeGoede, Physics Letters A 201, 221 (1995)

[308] J. F.Donges, R. V. Donner, J. Kurths, EPL (Europhysics Letters) 102, 10004 (2013)

[309] P. Guzik, J. Piskorski, T. Krauze, A. Wykretowicz, H. Wysocki, Biomedizinische Technik. Biomedical engineering 51, 272 (2006)

[310] M.B. Kennel, Phys. Rev. E 69, 056208 (2004)

[311] L. Lacasa, A. Nuñez, E. Roldán, J.M.R. Parrondo, B. Luque, The European Physical Journal B 85 (2012)

[312] A. Porta, S. Guzzetti, N. Montano, T. GnecchiRuscone, R. Furlan, A. Malliani, Time reversibility in short-term heart period variability, in Computers in Cardiology (2006), Vol. 33, pp. 77-80, 2006 Computers in Cardiology, CIC ; Conference date: 17-09-2006 Through 20-09-2006

[313] M. Zanin, A. Rodríguez-González, E. Menasalvas Ruiz, D. Papo, Entropy 20 (2018)

[314] W. Yao, W. Yao, D. Yao, D. Guo, J. Wang, Applied Physics Letters 116, 014101 (2020)

[315] J. Li, P. Shang, X. Zhang, Nonlinear Dynamics 96, 2637 (2019)

[316] R. Flanagan, L. Lacasa, Physics Letters A 380, 1689 (2016)

[317] J.A. Martín-Gonzalo, I. Pulido-Valdeolivas, Y. Wang, T. Wang, G. Chiclana-Actis, M.d.C. Algarra-Lucas, I. Palmí-Cortés, J. Fernández Travieso, M.D. Torrecillas-Narváez, A.A. Miralles-Martinez et al., Entropy 21, 868 (2019)

[318] J.H. Martínez, J.L. Herrera-Diestra, M. Chavez, Chaos: An Interdisciplinary Journal of Nonlinear Science 28, 123111 (2018)

[319] Y. Wenpo, J. Dai, M. Perc, J. Wang, D. Yao, D. Guo, Nonlinear Dynamics 100, 907 (2020)

[320] W. Yao, W. Yao, J. Wang, Physics Letters A 383, 1764 (2019)

[321] G.G. Malinetsky, Computer research and modeling 5, 315 (2013)

[322] T.U. Grund, The Journal of Mathematical Sociology 37, 131 (2013)

[323] R. Cohen, S. Havlin, Complex Networks: Structure, Robustness and Function (Cambridge University Press, 2010)

[324] G. Bianconi, EPL (Europhysics Letters) 111, 56001 (2015)

[325] S. Boccaletti, V. Latora, Y. Moreno, M. Chavez, D.U. Hwang, Physics Reports 424, 175 (2006)

[326] Dynamics of graph spectral entropy in financial crisis, in Socio-Economic Aspects of Economics and
Managment (Aspekt Publishing of Budget Printing Cente, 2015), pp. 227-234

[327] V. Soloviev, Bulletin of Cherkasy University 38, 67 (2015)

[328] V. Soloviev, V. Solovieva, A. Tuliakova, NeuroFuzzy Modeling Techniques in Economics 8, 3 (2019)

[329] V. Soloviev, V. Solovieva, A. Tuliakova, A. Hostryk, L. Pichl, CEUR Workshop Proceedings 2713, 53 (2020)

[330] S. Boccaletti, G. Bianconi, R. Criado, C. del Genio, J. Gómez-Gardeñes, M. Romance, I. SendiñaNadal, Z. Wang, M. Zanin, Physics Reports 544, 1 (2014), the structure and dynamics of multilayer networks

[331] M.C. Qian, Z.Q. Jiang, W.X. Zhou, Journal of Physics A: Mathematical and Theoretical 43, 335002 (2010)

[332] J. Birch, Ph.D. thesis (2015)

[333] F. Liu, N. Wang, D. Wei, The Open Cybernetics \& Systemics Journal 11, 36 (2017)

[334] W. Yan, E. van Tuyll van Serooskerken, PLOS ONE 10, 1 (2015)

[335] A. Johansen, O. Ledoit, D. Sornette, International Journal of Theoretical and Applied Finance 03, 219 (2000)

[336] M.D. Vamvakaris, A.A. Pantelous, K.M. Zuev, Physica A: Statistical Mechanics and its Applications 497, 41 (2018)

[337] M. Serafino, A. Gabrielli, G. Caldarelli, G. Cimini, Statistical validation of financial time series via visibility graph (2017), arXiv: 1710.10980 [q-fin. RM]

[338] C. Coquidé, J. Lages, D.L. Shepelyansky, Lecture Notes in Business Information Processing p. 208-219 (2019)

[339] T. Squartini, A. Gabrielli, D. Garlaschelli, T. Gili, A. Bifone, F. Caccioli, Complexity 2018, 1 (2018)

[340] L. Lacasa, V. Nicosia, V. Latora, Scientific Reports 5 (2015)

[341] L. Bargigli, G. di Iasio, L. Infante, F. Lillo, F. Pierobon, Quantitative Finance 15, 673 (2015)

[342] S. Li, S. Wen, Complexity 2017, 1 (2017)

[343] C. Stephen, Procedia Computer Science 53, 410 (2015)

[344] R.V. Donner, M. Small, J.F. Donges, N. Marwan, Y. Zou, R. Xiang, J. Kurths, International Journal of Bifurcation and Chaos 21, 1019 (2011)

[345] L. Lacasa, B. Luque, F. Ballesteros, J. Luque, J.C. Nuño, Proceedings of the National Academy of Sciences 105, 4972-4975 (2008)

[346] V. Soloviev, A. Tuliakova, Neuro-fuzzy modeling technologies in economics 5, 152 (2016)

[347] J. Iacovacci, L. Lacasa, Physical Review E 94 (2016)

[348] A. de la Concha, S. Martinez-Jaramillo, C. Carmona, in Complex Networks $\mathcal{E}$ Their Applications VI (Springer International Publishing, 2018), pp. 
$1135-1148$

[349] G. Colangelo, F.M. Ciurana, L.C. Bianchet, R.J. Sewell, M.W. Mitchell, Nature 543, 525-528 (2017)

[350] E.G. Hidalgo, Quantum econophysics (2006), arXiv:physics/0609245 [physics.soc-ph]

[351] V. Maslov, Math. Notes 72, 811 (2002)

[352] V. Soloviev, O. Serdiuk, Bulletin of Cherkasy University 1, 3 (2009)

[353] E. Benítez Rodríguez, L. Aguilar, Scientific Reports 8, 4010 (2018)

[354] L.A. Rozema, A. Darabi, D.H. Mahler, A. Hayat, Y. Soudagar, A.M. Steinberg, Phys. Rev. Lett. 109, 100404 (2012)

[355] M. Berta, M. Christandl, R. Colbeck, J.M. Renes, R. Renner, Nature Physics 6, 659-662 (2010)

[356] R. Prevedel, D.R. Hamel, R. Colbeck, K. Fisher, K.J. Resch, Nature Physics 7, 757-761 (2011)

[357] L. Landau, E. Lifshitz, Quantum Mechanics: NonRelativistic Theory, Course of Theoretical Physics, 3rd edn. (Butterworth-Heinemann, 1981)

[358] V. Soloviev, Y. Romanenko, Economic analog of Heisenberg uncertainly principle and financial crisis, in System analysis and information technology : 19-th International conference (ESC "IASA" NTUU "Igor Sikorsky Kyiv Polytechnic Institute", Kyiv, Ukraine, 2017), SAIT 2017, pp. 32-33

[359] V. Soloviev, S. Yevtushenko, V. Batareyev, CEUR Workshop Proceedings 2546, 87 (2019)

[360] S. Drozdz, J. Kwapien, P. Oswiecimka, Empirics versus rmt in financial cross-correlations (2007), arXiv:0711.0644 [q-fin.ST]

[361] F.J. Dyson, Journal of Mathematical Physics 3, 140 (1962)

[362] E.P. Wigner, Annals of Mathematics 53, 36 (1951)

[363] P.W. Anderson, Phys. Rev. 109, 1492 (1958)

[364] A. Lipton, A. Sardon, F. Schär, C. Schüpbach, From tether to libra: Stablecoins, digital currency and the future of money (2020), arXiv:2005.12949 [CS.CY]

[365] C. Karmakar, A. Khandoker, J. Gubbi, M. Palaniswami, Modified Ehlers' index for improved detection of heart rate asymmetry in Poincaré plot, in 36th Annual Computers in Cardiology Conference (CinC) (IEEE, 2009), Vol. 36, pp. $169-172$

[366] A. Porta, S. Guzzetti, N. Montano, T. GnecchiRuscone, R. Furlan, A. Malliani, Time reversibility in short-term heart period variability, in 2006 Computers in Cardiology (IEEE, 2006), Vol. 2006, pp. $77-80$

[367] A. Puglisi, D. Villamaina, EPL 88, 30004 (2009)

[368] A. Abhishta, R. Joosten, S. Dragomiretskiy, L. Nieuwenhuis, Impact of Successful DDoS Attacks on a Major Crypto-Currency Exchange, in 2019 27th Euromicro International Conference on Parallel, Distributed and Network-based Processing (PDP) (IEEE, United States, 2019), pp. 379-
384

[369] A.A. Kochkarov, S.D. Osipovich, R.A. Kochkarov, Revista ESPACIOS 41, 29 (2020)

[370] M. Conti, E. Sandeep Kumar, C. Lal, S. Ruj, IEEE Communications Surveys \& Tutorials 20, 3416-3452 (2018)

[371] M. Mirkin, Y. Ji, J. Pang, A. Klages-Mundt, I. Eyal, A. Juels, Bdos: Blockchain denial of service (2020), arXiv: 1912.07497 [cs.CR]

[372] M. Vasek, M. Thornton, T. Moore, Empirical Analysis of Denial-of-Service Attacks in the Bitcoin Ecosystem, in Financial Cryptography and Data Security. FC 2014. Lecture Notes in Computer Science, edited by R. Böhme, M. Brenner, T. Moore, M. Smith (Springer, 2014), Vol. 8438 of FC 2014, pp. 57-71

[373] U. Hacioglu, ed., Blockchain Economics and Financial Market Innovation, Contributions to Economics, 1st edn. (Springer, 2019), ISBN 978-3-03025275-5

[374] S. Nakamoto, Bitcoin: A peer-to-peer electronic cash system (2009), http://www.bitcoin.org/ bitcoin.pdf

[375] D. Aggarwal, G. Brennen, T. Lee, M. Santha, M. Tomamichel, Ledger 3 (2018)

[376] D. Sapaev, D. Bulychkov, F. Ablayev, A. Vasiliev, M. Ziatdinov, Quantum-assisted blockchain (2018), arXiv: 1802.06763 [quant-ph]

[377] O. Sattath, International Journal of Information Security 19, 291-302 (2020)

[378] L. Tessler, T. Byrnes, Bitcoin and quantum computing (2018), arXiv: 1711.04235 [quant-ph]

[379] L. Alessandretti, A. ElBahrawy, L.M. Aiello, A. Baronchelli, Complexity 2018, 1-16 (2018)

[380] N. Gandal, H. Halaburda, Games 7 (2016)

[381] T. Guo, A. Bifet, N. Antulov-Fantulin, 2018 IEEE International Conference on Data Mining (ICDM) (2018)

[382] H. Jang, J. Lee, IEEE Access 6, 5427 (2018)

[383] O. Sattarov, A. Muminov, C.W. Lee, H.K. Kang, R. Oh, J. Ahn, H.J. Oh, H.S. Jeon, Applied Sciences $10(2020)$

[384] D. Zhao, A. Rinaldo, C. Brookins, Cryptocurrency price prediction and trading strategies using support vector machines (2019), arXiv: 1911.11819 [q-fin.TR]

[385] T.R. Li, A.S. Chamrajnagar, X.R. Fong, N.R. Rizik, F. Fu, Frontiers in Physics 7, 98 (2019)

[386] W. Wei, Q. Zhang, L. Liu, Bitcoin transaction forecasting with deep network representation learning (2020), arXiv:2007.07993 [cS.SI]

[387] A.H.A. Othman, S. Kassim, R.B. Rosman, N.H.B. Redzuan, Journal of Revenue and Pricing Management 19, 314 (2020)

[388] S. McNally, J. Roche, S. Caton, Predicting the Price of Bitcoin Using Machine Learning, in 2018 26th Euromicro International Conference on Parallel, Distributed and Network-based Processing 
(PDP) (2018), pp. 339-343

[389] S.M. Raju, A.M. Tarif, Real-time prediction of bitcoin price using machine learning tech- niques and public sentiment analysis (2020), arXiv:2006.14473 [q-fin.ST]

[390] Hua, Yiqing, E3S Web Conf. 218, 01050 (2020) 